Vesa Hytönen

\title{
Handover performance in IEEE 802.16 mobile networks
}

Master's Thesis

in Information Technology

(Mobile systems)

December 5, 2009

\section{University of Jyväskylä}

Department of Mathematical Information Technology

$$
\text { Jyväskylä }
$$


Author: Vesa Hytönen

Contact information: vesa.a.hytonen@jyu.fi

Title: Handover performance in IEEE 802.16 mobile networks

Työn nimi: Yhteysvastuun vaihdon tehokkuus IEEE 802.16 mobiiliverkoissa

Project: Master's Thesis in Information Technology (Mobile systems)

Page count: 114

Abstract: In broadband wireless access network, continuous high speed data communication can be provided to mobile subscribers by well designed mobility management, where handover is a key element in maintaining air link to base station even in high velocity. When a mobile station changes its geographical position, it may also need to change its attachment point in the network in order to retain the quality of the connection. This is when a controlled handover is conducted. In the thesis, hard handover in IEEE 802.16 WiMAX network is studied by means of simulations in NS-2 network simulator. The studied handover type always causes a short interruption time before new connections are established with target base station. During this time user data cannot be transferred. Therefore, it is important to use handover optimization methods to decrease the interruption time, as well as avoid redundant handovers to minimize unnecessary connection drops. These topics are examined in this thesis.

Suomenkielinen tiivistelmä: Langattomassa laajakaistaverkossa liikkuville käyttäjille voidaan tarjota jatkuva nopea tietoliikenneyhteys hyvin suunnitellun liikkuvuuden hallinnan avulla, missä yhteysvastuun vaihto tarjoaa keinon säilyttää langaton yhteys tukiasemaan suuressakin nopeudessa. Mobiilipäätelaitteen vaihtaessa maantieteellistä sijaintiaan voi olla tarpeellista vaihtaa myös sen liityntäpistettä verkossa, jotta yhteyden laatu voitaisiin säilyttää. Tässä tilanteessa suoritetaan hallittu yhteysvastuun vaihto. Tutkielmassa tutkitaan hard handover -tyyppistä yhteysvastuun vaihtoa IEEE 802.16 WiMAX -verkossa NS-2-verkkosimulaattorin avulla. Tutkittu yhteysvastuun vaihdon tyyppi aiheuttaa aina lyhyen katkoksen ennen uusien yhteyksien luomista kohdetukiasemalle. Tämän katkoksen aikana ei voida siirtää käyttäjän dataa. Tästä syystä on tärkeää käyttää yhteysvastuun vaihdon optimointitapoja katkoksen lyhentämiseksi, ja välttää turhia yhteysvastuun vaihtoja yhteyskatkosten minimoimiseksi. Näitä aiheita käsitellään tässä tutkielmassa.

Keywords: information technology, Master's thesis, IEEE 802.16, WiMAX, handover, mobility, NS-2, simulation

Avainsanat: tietotekniikka, pro gradu -tutkielma, IEEE 802.16, WiMAX, yhteysvastuun vaihto, liikkuvuus, NS-2, simulointi 


\section{Contents}

$\begin{array}{ll}\text { Abbreviations } & 1\end{array}$

1 Introduction $\quad \mathbf{5}$

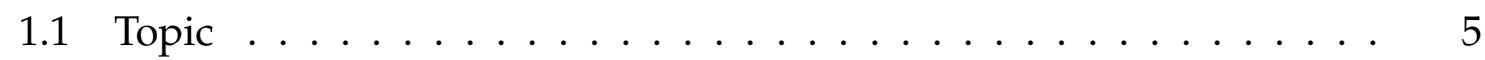

1.2 Research goals . . . . . . . . . . . . . . . . . 6

1.3 Research articles . . . . . . . . . . . . . . . . . 7

1.4 Document structure . . . . . . . . . . . . . . . 8

2 WiMAX technology 9

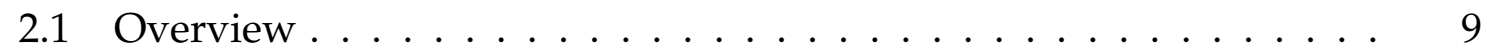

2.2 IEEE 802.16 Standards . . . . . . . . . . . . . . . . . 9

2.3 IEEE 802.16 protocol architecture . . . . . . . . . . . . . . . . . . . . . . . . . . . . . .

2.4 Physical $(\mathrm{PHY})$ Layer . . . . . . . . . . . . . . . . . . . . . . 12

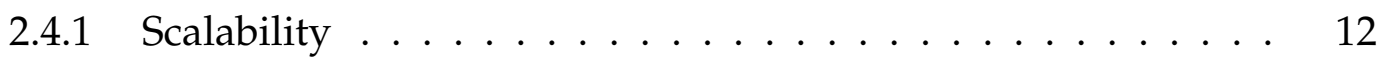

2.4.2 Adaptive modulation and coding (AMC) . . . . . . . . . . . 13

2.4 .3 Duplexing modes . . . . . . . . . . . . . . . . . . 13

2.4 .4 HARQ . . . . . . . . . . . . . . . . 15

2.5 Medium Access Control (MAC) Layer . . . . . . . . . . . . . . . . . 15

2.5.1 MAC convergence sublayer . . . . . . . . . . . . . . 15

2.5.2 MAC common part sublayer . . . . . . . . . . . . . . . 17

2.5.3 MAC security sublayer . . . . . . . . . . . . . 27

2.6 WiMAX Forum . . . . . . . . . . . . . . . 27

2.7 Network Working Group (NWG) . . . . . . . . . . . . . . . . 28

2.8 Network reference model . . . . . . . . . . . . . . . . 28

2.9 Network elements . . . . . . . . . . . . . . . . . . . . . . . 29

2.9 .1 Subscriber Station $(\mathrm{SS}) \ldots \ldots \ldots$

2.9.2 Access Service Network (ASN) . . . . . . . . . . . . . 30

2.9.3 Connectivity Service Network $(\mathrm{CSN})$. . . . . . . . . . . 35

2.10 Mobility in WiMAX . . . . . . . . . . . . . . . . 35

2.10 .1 Handover types . . . . . . . . . . . . . . 37

2.10 .2 Handover process . . . . . . . . . . . . . . . . . 39

2.10 .3 ASN-anchored mobility . . . . . . . . . . . . 42 
2.10.4 CSN-anchored mobility . . . . . . . . . . . . . . 44

2.10 .5 Roaming . . . . . . . . . . . . . . . . . . 44

3 Simulations $\quad 45$

3.1 Introduction . . . . . . . . . . . . . . . . . 45

3.2 Network Simulator $2 \ldots \ldots \ldots \ldots$. . . . . . . . . . . . 45

3.3 WiMAX NS-2 Extension (WINSE) . . . . . . . . . . . . 46

3.4 ASN-GW extension module . . . . . . . . . . . . . . . . . 48

3.4 .1 Core files . . . . . . . . . . . . . . . . . 48

3.4.2 File and class dependencies . . . . . . . . . . . . 50

3.5 Important simulator features $\ldots \ldots \ldots \ldots$. . . . . . . . . . 52

3.5.1 Scanning of neighbor BSs ................ 52

3.5.2 Handover module . . . . . . . . . . . . . . . . . . 52

3.5.3 Supported management messages . . . . . . . . . . . 53

3.5 .4 Handover timers . . . . . . . . . . . . . . . . 55

3.6 Simulation scenario . . . . . . . . . . . . . . . . 55

3.7 Simulation results . . . . . . . . . . . . . 58

$\begin{array}{lll}4 & \text { Further development ideas } & 69\end{array}$

5 Conclusions $\quad 71$

$\begin{array}{ll}\text { References } & 73\end{array}$

$\begin{array}{ll}\text { Appendices } & 77\end{array}$

Appendix A Handover performance in the IEEE 802.16 mobile networks $\quad 78$

Appendix B WINSE: WiMAX NS-2 Extension 86

$\begin{array}{lll}\text { Appendix C OTcl simulation script } & 104\end{array}$ 


\section{Abbreviations}

3GPP

$3 G$

$4 \mathrm{G}$

AAA

AAS

AK

AMC

ARQ

ASN-GW

ASN

ASP

BE

BRH

BR

BSID

BS

BWA

CBR

CDF

CID

CINR

CNR

CoA

CPE

CQICH

CSN

CS

DCD

DHCP

DL

DP

EP 3rd Generation Partnership Project

Third-generation

Fourth-generation

Authentication, Authorization and Accounting

Advanced Antenna System

Authorization Key

Adaptive Modulation and Coding

Automatic Repeat Request

Access Service Network Gateway

Access Service Network

Application Service Provider

Best Effort

Bandwidth Request Header

Bandwidth Request

Base Station Identifier

Base Station

Broadband Wireless Access

Constant Bit Rate

Cumulative Distribution Function

Connection Identifier

Carrier-to-Interference-and-Noise Ratio

Carrier-to-Noise Ratio

Care-of-Address

Customer Premises Equipment

Channel Quality Indication Channel

Connectivity Service Network

Convergence Sublayer

Downlink Channel Descriptor

Dynamic Host Configuration Protocol

Downlink

Decision Point

Enforcement Point 


\begin{tabular}{ll} 
ertPs & extended real-time Polling Service \\
ESF & Extended Subheader Field \\
ETSI & European Telecommunication Standards Institute \\
FA & Foreign Agent \\
FBSS & Fast Base Station Switching \\
FCH & Frame Control Header \\
FDD & Frequency Division Duplexing \\
FEC & Forward Error Correction \\
FFSH & Fast-feedback Subheader \\
FFT & Fast Fourier Transform \\
FSH & Fragmentation Subheader \\
FTP & File Transfer Protocol \\
GMH & Generic MAC Header \\
GMSH & Grant Management Subheader \\
GPCS & Generic Packet Convergence Sublayer \\
HARQ & Hybrid Automatic Repeat Request \\
HA & Home Agent \\
HHO & Hard Handover \\
HO & Handover/Handoff \\
IEEE & Institute of Electrical and Electronics Engineering \\
IETF & Internet Engineering Task Force \\
IE & Information Element \\
IMS & IP Multimedia Subsystem \\
IMT & International Mobile Telecommunications \\
IP(v4/v6) & Internet Protocol (version 4/version 6) \\
ISD & Inter-Station Distance \\
ISF & Initial Service Flow \\
ISP & Internet Service Provider \\
ITU & International Telecommunication Union \\
LAN & Local Area Network \\
LSB & Least Significant Bit \\
LTE & Long Term Evolution \\
MAC & Medium Access Control \\
MAN & Metropolitan Area Network \\
MBS & Multicast and Broadcast Service \\
MCS & Modulation and Coding Scheme \\
MDHO & Macro Diversity Handover \\
& \\
\hline
\end{tabular}




\begin{tabular}{|c|c|}
\hline MIMO & Multiple-Input-Multiple-Output \\
\hline MIP & Mobile IP \\
\hline MMR & Mobile Multihop Relay \\
\hline $\mathbf{M M}$ & Mobility Management \\
\hline MR-BS & Multihop Relay Base Station \\
\hline MRS & Mobile Relay Station \\
\hline MR & Multihop Relay \\
\hline MSB & Most Significant Bit \\
\hline MS & Mobile Station \\
\hline NAP & Network Access Provider \\
\hline NRM & Network Reference Model \\
\hline nrtPS & non-real-time Polling Service \\
\hline NSP & Network Service Provider \\
\hline NWG & Network Working Group \\
\hline OFDMA & Orthogonal Frequency Division Multiple Access \\
\hline OFDM & Orthogonal Frequency Division Multiplexing \\
\hline OSI & Open Systems Interconnection \\
\hline PCC & Policy and Charging Control \\
\hline PDU & Packet Data Unit \\
\hline PHS & Payload Header Suppression \\
\hline PKM & Privacy and Key Management Protocol \\
\hline PMIP & Proxy-Mobile IP \\
\hline PMP & Point-to-Multipoint \\
\hline PM & Poll-me bit \\
\hline PoA & Point of Attachment \\
\hline PPP & Point-to-Point Protocol \\
\hline PSH & Packing Subheader \\
\hline PSTN & Public Switched Telephone Network \\
\hline QoS & Quality of Service \\
\hline RFC & Request For Comments \\
\hline $\mathbf{R P}$ & Reference Point \\
\hline RRC & Radio Resource Controller \\
\hline RRM & Radio Resource Management \\
\hline RSSI & Received Signal Strength Indication \\
\hline RS & Relay Station \\
\hline RTG & Receive/transmit Transition Gap \\
\hline rtPS & real-time Polling Service \\
\hline
\end{tabular}




\begin{tabular}{ll} 
SAP & Service Access Point \\
SC & Single Carrier \\
SDU & Service Data Unit \\
SFA & Service Flow Authorization \\
SFID & Service Flow Identifier \\
SFM & Service Flow Management \\
SF & Service Flow \\
SNMP & Simple Network Management Protocol \\
SNR & Signal-to-Noise Ratio \\
SOFDMA & Scalable Orthogonal Frequency Division Multiple Access \\
SS & Subscriber Station \\
TCP & Transmission Control Protocol \\
TDD & Time Division Duplexing \\
TDMA & Time Division Multiple Access \\
TGm & Task Group m \\
TISPAN & Telecommunications and Internet converged Services and Proto- \\
& cols for Advanced Networking \\
TLV & Type/Length/Value \\
TTG & Transmit/receive Transition Gap \\
UCD & Uplink Channel Descriptor \\
UDP & User Datagram Protocol \\
UE & User Equipment \\
UGS & Unsolicited Grant Service \\
UL & Uplink \\
VCI & Virtual Channel Identifier \\
VoIP & Voice over Internet Protocol \\
VPI & Worldwide Interoperability for Microwave Access \\
WiMAX & Sirtual Path Identifier \\
\hline
\end{tabular}




\section{Introduction}

\subsection{Topic}

This thesis handles WiMAX technology from the air interface perspective as well as from the access network point of view and the mobility support they provide. Main concentration is on handover (handoff) performance. IEEE 802.16e-2005 standard [1], or 'Mobile WiMAX', defines wireless network access for fixed and mobile users by specifying Medium Access Control layer (MAC) functionality as well as Physical layer (PHY) that supports single carrier (SC), Orthogonal Frequency Division Multiplexing (OFDM) and Orthogonal Frequency Division Multiple Access (OFDMA). The Mobile WiMAX standard expands previously released IEEE 802.162004 [2] specification to support mobility services for subscriber stations (SS) moving at vehicular speeds and updates the MAC and PHY layer mechanisms with new and improved features [1]. 802.16j standard [3] expands the mobility support further by introducing multihop relays (MR) to enhance coverage and performance in WiMAX network. WiMAX technology beyond the air interface is being developed and defined by the WiMAX Forum, an organization consisting of operators and component and equipment manufacturers [7].

The next-generation wireless systems will acquire increasingly more market area from the currently dominant $3 \mathrm{G}$ and $3.5 \mathrm{G}$ systems as the bandwidth demand grows. Operators are pushed to offer fast, flexible and reliable connections for subscribers. While fixed and nomadic wireless access has been available for some time now, full mobility supporting networks reaching very high bandwidth are knocking on the door. Especially real-time data services, for instance VoIP and video streaming, depend on continuous, high quality networking. Mix it with high speed mobility up to $120 \mathrm{~km} / \mathrm{h}$ and the goal for maintaining good quality of service (QoS) becomes demanding from the technical point of view. One of the key issues in ensuring the network to be able to handle such cases is proper mobility management (MM), where well designed handover procedures play a big role. 


\subsection{Research goals}

The objective of this thesis is to study behavior and performance of the WiMAX network during handovers on the one hand from the air interface point of view and on the other hand from the access network perspective. Impact of basic handover related parameters are being observed to find out preferable values in order to increase the handover performance.

Usually a handover is performed when the signal quality level of a serving base station (BS) or a relay station (RS) is deteriorated below the signal quality level of another BS/RS during mobile station's (MS) movement from the coverage area of the serving BS/RS to the coverage area of another BS/RS, although there exist many additional handover triggering mechanisms. In the simulation part of this thesis only signal strength measurements are used for handover initiation, therefore it is studied quite thoughtfully. Nevertheless, other mechanisms are explained also at theoretical level. [21][33]

A decision when to perform a handover is a bit problematic since the signal levels tend to have fluctuation due to wireless propagation factors, such as shadowing and fast fading. Therefore overly sensitive handover triggering may result in increased connection drops, signaling overhead and possibly a so called ping-pong effect in a circumstance where two or more cells provide almost equally good conditions for the MS to enter the network. In such case a MS perpetually may switch its traffic anchor point in the network. However, handovers performed too sparsely will cause prolonged reduction of the signal power level prior to a handover, thus leading to a bigger amount of dropped packets and worse throughput performance. [33]

One solution to improve the network behavior regarding handovers and to find a trade-off between the two situations explained above is by adjusting handover thresholds and timers. Figure 1.1 depicts an example scenario of a handover taking place. The scenario contains two signal strength levels from two different base stations. In this simple example adjustable values would be hysteresis margin $h$ and Time-to-trigger timer $T_{h o}$. The first one determines how much the signal strength of the nearby BS has to exceed the signal strength of the serving BS in order to initiate a handover. The latter tells how long such a condition should remain before the handover triggering. [8]

There are four important action points in the figure, $T_{1}-T_{4}$. At times $T_{1}$ and $T_{3}$ the signal strength of the second base station (BS 2) exceeds the hysteresis margin $h$ and the handover trigger timer is started. At $T_{2}$ the SS notices that the signal strength 


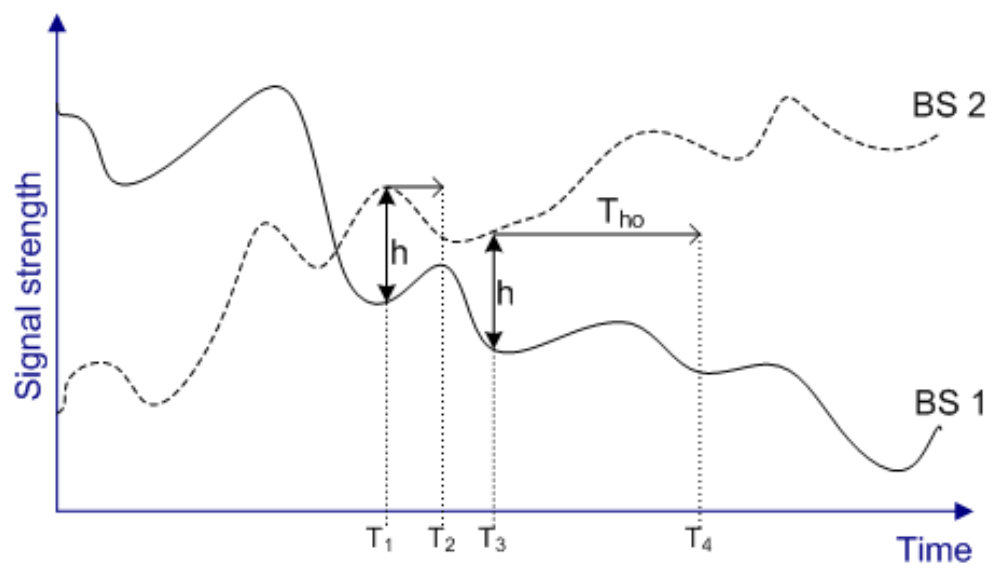

Figure 1.1: Signal levels from two BSs.

difference is below the $h$ so the handover timer is halted. At $T_{4}$ the handover trigger timer $T_{h o}$ expires, meaning it is time to perform a handover to the BS 2 which then becomes the new serving BS.

The method described is an example of a simple handover algorithm. In reality the signal strengths of BSs would have much more variance than shown in the figure due to fast fading. This makes it more difficult to determine values for the $h$ and the $T_{h o}$ that would apply well for versatile conditions. Instead of having a simple algorithm that compares the signal strength levels from each BS/RS and triggers a handover according to the comparisons, a bit more sophisticated one might provide a better performance.

\subsection{Research articles}

Two research articles are included in the appendices of this document. The author of the thesis is an author also in both of these articles.

During the study of the topic and writing process of the thesis one research article was written and submitted to SIMUTools 2010, the Third International Conference on Simulation Tools and Techniques. The article, 'Handover performance in the IEEE 802.16 mobile networks', presents basically same theory and results to those of this thesis. Therefore same results and explanations can be found from both documents. The conference will be held in Malaga, Spain, on March 15-19, 2010. Information on possible acceptance of the article will be given on January 12, 2010, at the latest. Complete version of the handover article is attached in Appendix A.

A journal publication, 'WINSE: WiMAX NS-2 Extension', can be found in Ap- 
pendix B. It describes features of the WINSE module, which is used for obtaining the results for this thesis and the handover article. The WINSE journal publication was submitted for the Special Issue of Simulation: Software Tools, Techniques and Architectures for Computer Simulation.

\subsection{Document structure}

The thesis is divided into four larger parts. Section 2 introduces the reader to the WiMAX technology from the organizational and technical point of view. This will help understanding WiMAX as a whole. Main concentration is on abilities of the MAC and PHY layers and also introduction of features of the access network is provided. Section 2.10 focuses more closely on mobility in WiMAX. Section 3 introduces the hands-on part of the thesis. It contains explanations for all the tools used as well as simulation scenarios and gathered results. The last part of the thesis includes conclusions and suggestions for further work. 


\section{WiMAX technology}

\subsection{Overview}

WiMAX is a digital next-generation communication technology that provides broadband wireless access (BWA) for fixed, nomadic, portable and mobile subscribers in line-of-sight (LOS) and non-line-of-sight (NLOS) environment with a BS. The IEEE 802.16 supports broadband wireless Internet access up to $50 \mathrm{~km}$ distance for fixed and 5-15 km distance for mobile subscribers. WiMAX provides an adaptive endto-end architecture that employs SC, OFDM and OFDMA physical layers for multiuser accessibility. Peak data rate of $72 \mathrm{Mbps}$ can be reached for downlink, although in reality the transfer data rate will be at most around $15 \mathrm{Mbps}$ with the currently latest standard release. Functionality of subscriber station, base station and air interface between them is defined in IEEE's 802.16 standard, whereas the access network operability is quantified by the WiMAX Forum and its working groups. [4][8]

This section is logically divided into three parts. First IEEE 802.16 standards and features are introduced, after which the focus is on WiMAX Forum and its Network Working Group (NWG) as well as on the specification of the WiMAX access network. After that, mobility in WiMAX is presented. As for the references, mostly standards already released and in draft stage are used in the section due to its technical orientation.

\subsection{IEEE 802.16 Standards}

The development work around the 802.16 telecommunication system and later WiMAX was initiated by the IEEE by setting up the 802.16 working group in 1999 under the IEEE 802.16 LAN/MAN Standards Committee. Table 2.1 describes the standard evolution of 802.16 from its beginning to yet to published WiMAX specifications.

Development towards the Mobile WiMAX started from the IEEE 802.16 Broadband Wireless Metropolitan Area Network (WirelessMAN) standard finalized in 2001 and published in 2002. It was to provide a last mile air interface specification for fixed subscribers in LOS only condition between subscriber station and base station. In the next two years it was followed by amendments $802.16 \mathrm{a}$ and $802.16 \mathrm{c}$ that 
Table 2.1: Family of IEEE 802.16 standards.

\begin{tabular}{|c|c|c|c|}
\hline IEEE Std & $\begin{array}{l}\text { Standard/ } \\
\text { Amendment }\end{array}$ & Information & Status \\
\hline $802.16-2001$ & Standard & $\begin{array}{l}\text { The basic } 802.16 \text { standard providing air interface for fixed BWA } \\
\text { systems in } 10-66 \mathrm{GHz} \text { band. }\end{array}$ & Obsolete \\
\hline $802.16 a-2003$ & Amendment & $\begin{array}{l}\text { Enhanced MAC layer functionality and extended the physical } \\
\text { layer to operate in 2-11 GHz band and in NLOS condition. }\end{array}$ & Obsolete \\
\hline $802.16 \mathrm{~b}$ & Amendment & $\begin{array}{l}\text { This amendment was meant to enhance the original } 802.16 \\
\text { project by extending it to licence-exempt frequency bands. It was } \\
\text { later merged into the } 802.16 \text { a project. }\end{array}$ & Obsolete \\
\hline $802.16 c-2002$ & Amendment & $\begin{array}{l}\text { The amendment was to provide detailed system profiling and } \\
\text { performance analysis when operating in } 10-66 \mathrm{GHz} \text { band. }\end{array}$ & Obsolete \\
\hline $802.16-2004$ & Standard & $\begin{array}{l}\text { Before approval was known as } 802.16 \mathrm{~d} \text {. The standard consoli- } \\
\text { dates the } 802.16 \text { and its amendments by covering LOS and NLOS } \\
\text { applications in } 2-66 \mathrm{GHz} \text { band. It is the first WiMAX standard } \\
\text { providing OFDM with } 256 \text { carriers. }\end{array}$ & Active \\
\hline $802.16 \mathrm{e}-2005$ & Amendment & $\begin{array}{l}\text { The } 802.16 \text { introduces nomadic and mobile support for end- } \\
\text { users with } 2-6 \mathrm{GHz} \text { frequency band. The mobility support in- } \\
\text { cludes e.g. hard handover and two types of soft handovers: } \\
\text { MDHO and FBSS. }\end{array}$ & Active \\
\hline $802.16 f-2005$ & Amendment & This amendment contains the management information base. & Active \\
\hline $802.16 \mathrm{~g}-2007$ & Amendment & $\begin{array}{l}\text { The amendment introduces management plane procedures and } \\
\text { services. }\end{array}$ & Active \\
\hline $802.16 \mathrm{~h}$ & Amendment & $\begin{array}{l}\text { This amendment to the IEEE Std. } 802.16 \text { will provide improved } \\
\text { coexistence mechanisms for licence-exempt operation. }\end{array}$ & $\begin{array}{l}\text { Under develop- } \\
\text { ment }\end{array}$ \\
\hline $802.16 \mathrm{i}$ & Amendment & $\begin{array}{l}\text { Withdrawn project on management plane procedures and ser- } \\
\text { vices. The content was moved into } 802.16 \text { Rev } 2 \text { draft. }\end{array}$ & Terminated \\
\hline $802.16 \mathrm{k}-2007$ & Amendment & Presents bridging mechanisms of 802.16 . & Active \\
\hline $802.16-2009$ & Standard & $\begin{array}{l}\text { This is the next } 802.16 \text { standard that should be available in De- } \\
\text { cember 2009. It was developed under the draft title 'P802.16Rev2' } \\
\text { and it consolidates previously published standards. }\end{array}$ & Approved \\
\hline $802.16 j-2009$ & Amendment & $\begin{array}{l}\text { An amendment to } 802.16-2009 \text { that introduces the multihop relay } \\
\text { specification. It will be published along with the } 802.16-2009 \text {. }\end{array}$ & Approved \\
\hline
\end{tabular}

detailed and extended the MAC and PHY features. [34]

The 802.16-2004 standard [2] was completed in 2004 and superseded the IEEE 802.16 and its amendments. It specified the air interface including MAC and PHY layer functionality for broadband wireless access (BWA) systems. The standard introduced some new MAC features, such as automatic repeat request (ARQ) mechanism and mesh topology. It also supported SC, OFDM and OFDMA physical layers and an ability for NLOS connections. Generally the 802.16-2004 standard is referred to as the first one adopting the name 'WiMAX', a name concocted after the WiMAX Forum. [2][7]

The first 802.16 standard supporting real mobility of subscriber stations is the 802.16e-2005 [1], which is an amendment for the 802.16-2004. It was completed and approved in the end of 2005 and published in 2006. It is not backward com- 
patible with the 802.16-2004, so the equipment need to be updated to support it. The 802.16e-2005 is referred to as 'Mobile WiMAX' since it presented all the needed factors for mobility of subscriber stations moving from air interface of one BS to air interface of another BS. The standard added support for sub-channelization as well as for SOFDMA with up to 2048-bit Fast Fourier Transform (FFT) which allows usage of dynamical channel bandwidths from $1.25 \mathrm{kHz}$ to $20 \mathrm{kHz}$. Other notable remarks from the 802.16e are power management and support for 'smart' antenna systems, such as Multiple-Input-Multiple-Output (MIMO), beamforming and Advanced Antenna Systems (AAS). By the new standard WiMAX took a big step on its way to a successful cellular wireless technology. From the mobility point of view the 802.16e allowed a mobile equipment to perform upper layer controlled handovers (including normal 'hard' handover and two types of soft handovers, namely fast base station switching (FBSS) and macro diversity handover (MDHO)), while earlier the only possible 'handover' convention was to exit from serving network and perform a network entry to the new one without handover preparation signaling or mobile station context exchange within the access network. [1]

Currently the IEEE 802.16e-2005 is most widely used among the WiMAX standard family. The next upcoming entrant will be the 802.16-2009 that is being developed under the draft name 'P802.16Rev2'. According to [6] it will be published in December 2009 as well as its remarkable amendment 802.16j which introduces multihop relay specification to the WiMAX technology. It should enhance the network coverage and performance by presenting relay stations (RS), simplified BS-like units via which the SS is able to attach to the multihop relay base station (MR-BS) and from there to the backbone network. [3][8]

$802.16 \mathrm{~m}$ is the newest draft standard developed by the IEEE 802.16's Task Group $\mathrm{m}$ (TGm) and is planned to be published in 2010. It will enhance the IEEE 802.16 WirelessMAN-OFDMA specification to meet IMT-Advanced requirements. IMTAdvanced is a concept of fourth-generation (4G) telecommunication systems and it is developed by the International Telecommunication Union (ITU). IMT-Advanced is the next step from IMT-2000 that is a set for 3G telecommunication systems. IMTAdvanced should offer constantly high data rates, meaning $100 \mathrm{Mbps}$ in high mobility and even 1 Gbps in low mobility applications. [30][31]

\subsection{IEEE 802.16 protocol architecture}

The 802.16 standard defines a protocol architecture model quite similar to many other protocol stacks that comprise various layers. The functions defined by the ar- 
chitecture are implemented in stations attending the air interface traffic. Figure 2.1 depicts the 802.16 protocol stack. It includes a Medium Access Control (MAC) layer divided into a convergence sublayer, a common part sublayer and a security sublayer, and a Physical (PHY) layer. In comparison to Open Systems Interconnection (OSI) reference model the MAC layer corresponds to OSI’s Data Link layer. [8]

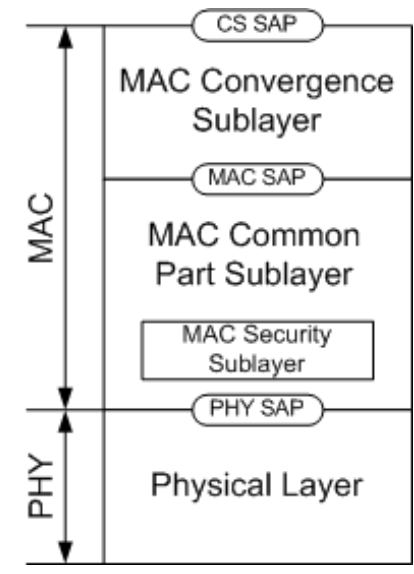

Figure 2.1: WiMAX protocol stack.

Each layer of the stack performs data processing and forwards a data unit to a lower or upper layer depending on the equipment processing the data being the sender or the receiver. [8]

\subsection{Physical (PHY) Layer}

Before transmission to the wireless link at the sender side, or right after reception at the receiver side, packets go through the 802.16 PHY layer. It performs operations, such as channel coding and interleaving, before passing on the packet. Some of the main features and functions of the 802.16 PHY layer are introduced in this section.

\subsubsection{Scalability}

The OFDMA using 128-, 512-, 1024- or 2048-bit Fast Fourier Transform (FFT) is able to support dynamically scaling channel bandwidths of $1.25 \mathrm{MHz}, 5 \mathrm{MHz}, 10 \mathrm{MHz}$ and $20 \mathrm{MHz}$, respectively. [8] 


\subsubsection{Adaptive modulation and coding (AMC)}

Modulation and coding scheme can be changed for each burst separately. Different modulation and coding schemes offer either robust or efficient network access, thus offering stable QoS in varying conditions. Three modulation schemes are shown in figure 2.2. The 64QAM offers highest bandwidth whereas the QPSK modulation is the most robust, therefore offering highest distance from the serving station. Also BPSK modulation can be used but it is not mandatory for UL or DL connections. [8]

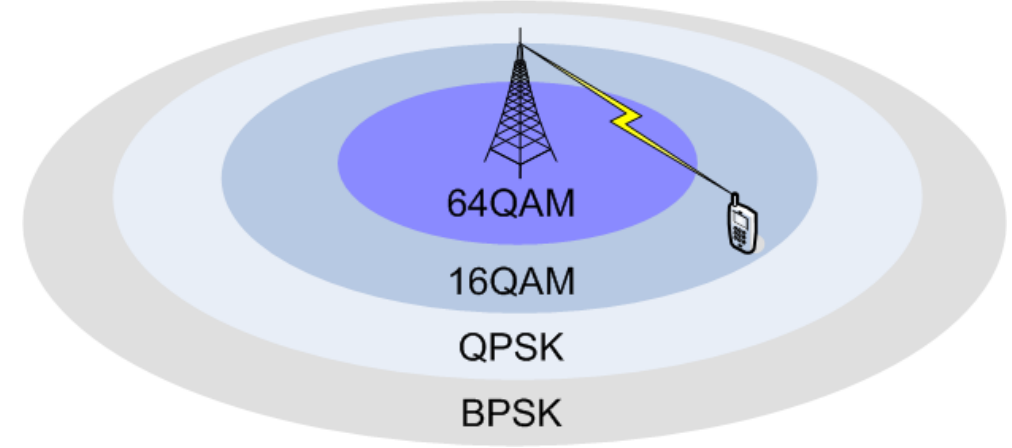

Figure 2.2: Adaptive modulation and coding.

In addition to different modulations there are a few coding rates used also to provide flexible networking. Depending on the Carrier-to-Noise Ratio (CNR) a coding rate of $1 / 2,3 / 4$ or $5 / 6$ can be used. [1]

\subsubsection{Duplexing modes}

WiMAX supports time division duplexing (TDD), full-duplex frequency division duplexing (F-FDD) and half-duplex frequency division duplexing (H-FDD). The usage of different duplexing modes affect the data transmission convention. In WiMAX user data is transmitted inside frames that consist of uplink and downlink subframes separated from each other by a TTG guard interval in TDD or different frequencies in FDD. Both DL and UL frames store user information into bursts which applies for both TDD and FDD frame. Depending on the duplexing mode being OFDMA/OFDM TDD, F-FDD or H-FDD, the frame structure changes. In addition to these, 802.16j defines a new frame format for communication between a multihop relay base station (MR-BS) and a RS. [8][3]

In TDD the DL subframe and the UL subframe are transmitted in the same channel consecutively which is useful when working with limited bandwidth resources. In addition, TDD's dynamically adjustable DL/UL ratio allows it to be used with 
both symmetric and asymmetric traffic. In FDD the DL and UL subframes are transmitted in separate concurrent channels using different frequencies. The difference between F-FDD and H-FDD is that in F-FDD a user is able to transmit and receive at the same time while in H-FDD user can either transmit or receive at given time but not do both simultaneously. [8]

TDD is mostly used where WiMAX network is deployed. Main reasons for the popularity of TDD are flexibility, cost efficiency and high spectral efficiency [5]. Taking a closer look into a TDD frame reveals how user information is divided within a frame (figure 2.3). The smallest time-frequency divided element in WiMAX is called a slot, which constitutes one sub-channel and one, two or three OFDM/OFDMA symbols. All the data regions in a frame are composed from sequential slots. [8]

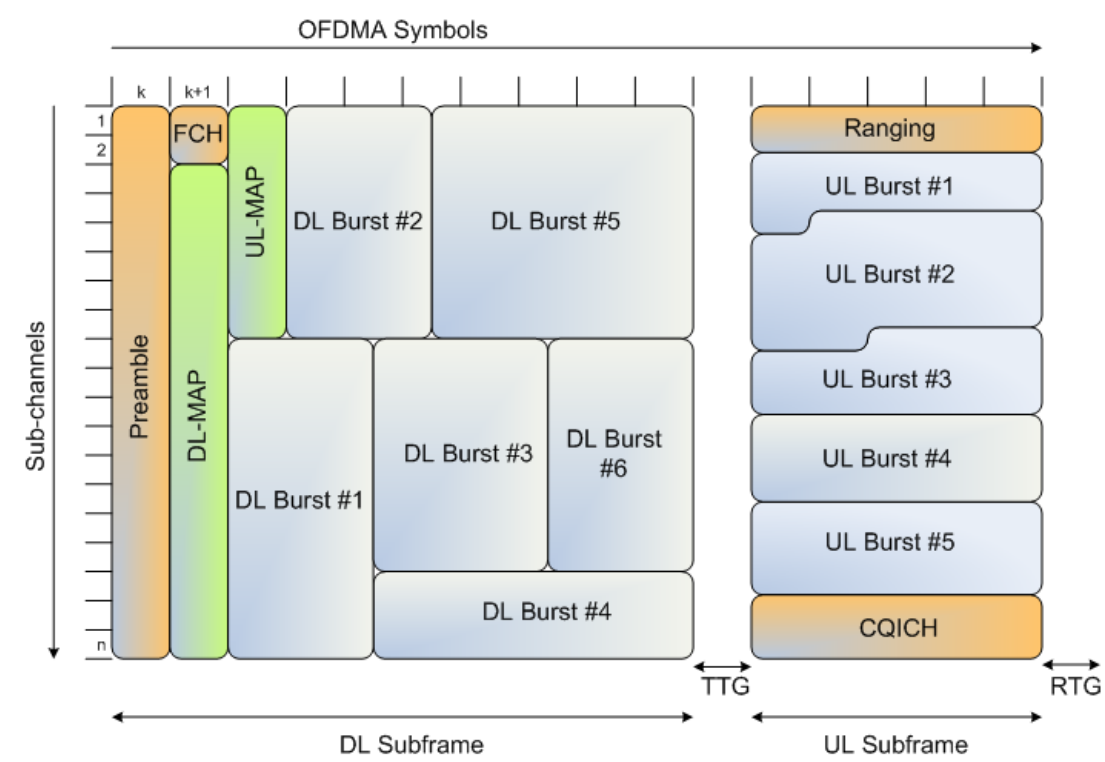

Figure 2.3: TDD frame structure.

A TDD DL subframe starts with a downlink preamble field that is used for synchronization and Carrier-to-Interference-and-Noise Ratio (CINR) and Received Signal Strength Indication (RSSI) measurements. The frame start preamble is followed by a Frame Control Header (FCH) that includes, for example, information on MAP sizes and usable subcarriers. The DL-MAP and UL-MAP fields contain Information Elements (IE) that tell the distribution of user data bursts (burst profile for each user) within DL and UL subframes. The MAP information may point to current frame or to subsequent frames, and the implementation of which frame they pertain is determined by the network vendor. Keeping in mind that the modulation can be changed on a per-burst basis, and that it is important to maintain the MAP information readable, a very robust MCS is usually used for MAP messages. In DL subframe actual 
user data is stored in DL bursts, where one burst is reserved for one user CID. Burst profile rules defined in the MAP field is applied on each burst. Burst profiles are used for link adaptation and in DL they may affect, in addition to the MCS, the DL Forward Error Correction (FEC) code type. [8]

UL subframe begins with a ranging subchannel which is used by SSs for closedloop time, frequency and power adjustments and also for carrying bandwidth requests. The UL bursts contain user data transmitted from SS to BS. The CQI channel $(\mathrm{CQICH})$ is used for transmission of channel quality information feedback. [11]

In TDD a TTG time interval separates DL and UL subframes. During the TTG gap the BS turns off its transmitter and switches from transmit (Tx) to receive (Rx) mode. Receive/transmit transition gap (RTG) is placed after the UL subframe to distinguish current frame from the following one. During the RTG BS switches from the Rx mode back to the Tx mode. [8]

\subsubsection{HARQ}

Hybrid-ARQ is a mechanism for packet retransmissions that is premised on packet reception acknowledgments. It works on the PHY layer and with FEC it provides fast retransmissions of PDUs. A negative side of HARQ is its cost of implementation. [4]

\subsection{Medium Access Control (MAC) Layer}

The MAC layer performs a great deal of the WiMAX specific operations which relate to connection and traffic management, security and quality of service. Although this section depicts MAC layer functionality and that MAC layer operations impact mostly air interface communication, there is a great synergy between the MAC layer functions and influence of access network on overall network performance. Therefore it is advisable to keep in mind the affect of both of them while reading this section for understanding the whole picture of the WiMAX technology.

\subsubsection{MAC convergence sublayer}

The convergence sublayer (CS) resides on top of the protocol stack. The CS has five major tasks to perform [8]:

- Receive higher layer packet data units (PDUs) from the higher layer. Above the CS layer resides the network layer at which works, for instance, IP, ATM 
or Ethernet transmission technology.

- Perform classification of higher layer PDUs. The classification method depends on which CS specification is used. Three available specifications are explained later in this section.

- Process the higher level PDUs based on the classification if required. This includes, for example, Payload Header Suppression (PHS).

- Deliver CS PDUs to correct MAC Service Access Point (SAP) which works as a waypoint to a lower sublayer.

- Receive CS PDUs from the peer entity.

The Payload Header Suppression (PHS) is an optional feature used for decreasing overhead by means of removing the repetitive portion from the payload headers at the transmitter. Then, the receiver restores the header and reinserts the repetitive part back to the header information.

The [8] defines three CS specifications: ATM CS, packet CS and Generic Packet CS. The reason for several specifications is that the service specific CSs map the 802.16 MAC onto a standard network interface, thus making the MAC versatile and compatible to multiple network technologies.

The ATM CS receives ATM cells from higher layer, performs classification, and PHS if needed, and forwards the CS PDUs to the MAC SAP. The ATM connection is identified by the Virtual Path Identifier (VPI) and Virtual Channel Identifier (VCI) pair which is used for mapping the CS data units to an appropriate connection identifier (CID) used at lower MAC sublayers. The classification and CID mapping are part of the basic QoS enhancement mechanisms in WiMAX. [8]

The packet CS is the second type of specified CSs. It provides a support for packet-based protocols, such as IP(v4/v6), Point-to-Point Protocol (PPP) and Ethernet (802.3). The packet CS may perform PHS operations which is again optional. Mandatory tasks for this CS are MAC Service Data Unit (SDU) classification, delivery of CS PDUs to the MAC SAP and receipt of CS PDUs from the peer MAC SAP. Whereas the ATM CS uses, for instance, VPI/VCI values for classification, the packet CS maps MAC SDUs to a transport connection according to packet-specific information, such as destination IP address. The classification produces reference to a CID value and creates an association to used service flow characteristics. [4][8]

The Generic Packet Convergence Sublayer (GPCS) is independent from higherlayer networking protocols which allows it to support multiple packet-based proto- 
cols over 802.16 air interface. It is not designed to replace or re-define the ATM CS or the packet CS. The GPCS was introduced in the 802.16g standard. [8][32]

\subsubsection{MAC common part sublayer}

The MAC common part sublayer plays essential role between the network layer and the physical layer by performing multiple tasks related to traffic management and QoS and forms the 'heart' of the 802.16 technology. Next some of the main areas of responsibility of the common MAC are described.

\section{Point-to-Multipoint (PMP)}

WiMAX is a connection oriented technology which means that all data services are mapped to a connection between the SS and the BS. In other words, a connection between the entities must be established before any unicast traffic can be transmitted. The DL direction from BS to SS operates on a Point-to-Multipoint (PMP) basis in which a central BS is able to provide independent, frequency-separated sectors and serve multiple SSs located in these sectors. [8]

DL traffic is usually broadcasted so all the SSs that are served by the BS are able to receive the data. DL-MAP located in the beginning of frame indicates which portion of the DL frame is addressed to a specific connection. The UL transmissions from the SS to the BS are performed on request/allocation basis. This way the BS together with the core network is able to control the UL traffic, thus managing also QoS and decreasing air interface congestion. [8]

\section{Connections}

Connections between network entities are used for message and data transmission. They are divided into management connections and transport connections which carry management messages and user data, respectively. The UL and DL connections are treated in pairs, so initializing, modifying or deleting a connection affects the opposite direction one the same way. Connections are identified by 16-bit CID values, regardless of the type of the connection. [8]

A pair of basic management connections is created when a SS enters the network, as well as UL and DL primary management connections. Optionally, also secondary management connections may be established. The basic management connections are used for transportation of MAC management messages that demand small delays. This includes many types of QoS related messages that try to maintain the 
link between SS and BS, such as ARQ, handover and network entry messages. The primary management connections carry more delay-tolerant messages, for example transport connection creation, modification of deletion related messages. The optional secondary management connection is used for transmitting standard-based messages from BS to SS. These include, e.g. Dynamic Host Configuration Protocol (DHCP) and Simple Network Management Protocol (SNMP) messages. [8]

Transport connections are used for carrying application data between entities. Data is stored into MAC PDUs during transmission. A transport connection is identified by a CID similarly to management connections. A transport connection has an association to a service flow which allows applying QoS on a per-connection basis. [8]

\section{MAC PDU}

As illustrated in figure 2.4, each MAC PDU consists of header portion, an optional payload which contains SDU information and possibly a CRC field. The common part MAC sublayer may perform packing or fragmentation of MAC SDUs. When packing is used, small SDUs are concatenated into one PDU. With fragmentation one SDU may be split into several PDUs before passing them to PHY layer. [8]

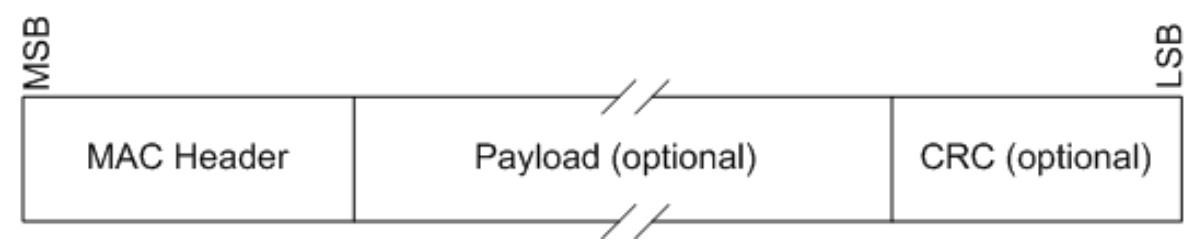

Figure 2.4: MAC PDU structure.

There are two types of MAC headers: a generic MAC header (GMH) used with PDUs containing management messages or user data and a bandwidth request header (BRH) for SS to request UL bandwidth from BS. The GMH is the only header type used in DL transmissions, whereas both GMH and BRH are used in UL. [8]

The generic MAC header contains information on payload encryption and CRC, a connection identifier, length of the PDU and information on possible subheaders and special payload types such as ARQ feedback. The 802.16 standard defines five subheader types for generic MAC header: packing subheader (PHS), fragmentation subheader (FSH), Fast-feedback allocation subheader (FFSH), Grant Management subheader (GMSH) and extended subheader. More information on these subheader types can be found in [1]. 
Bandwidth Request Header (BRH) is the second main header type in addition to GMH. More about bandwidth request and allocation mechanisms is explained in the next section.

\section{Bandwidth requests and allocations}

Bandwidth requests are used by SSs to inform BSs about need for UL bandwidth allocation. BRs can be transmitted as stand-alone BRHs or piggybacked within a normal PDU. Scheduler of the BS may allocate resources on per-SS basis, and it is the SS that decides how to share the bandwidth for each of its connections. The bandwidth requesting customs depend on QoS parameters, including UL scheduling type. For example, UGS connections do not need to request for bandwidth (unless it enables a specific poll-me (PM) bit), since it is allocated resources on a regular basis. For lower priority connections there are unicast polling and contention-based methods that allow SSs to transmit BRs to BSs. [4][8]

Unicast polling is one of the mechanisms that a BS uses to allocate SSs bandwidth for their bandwidth requests. In unicast polling BS designates a bandwidth grant for each SS in UL-MAP individually. [8]

Multicast and broadcast polling for grouped SSs can be used instead of unicast polling if there is not enough resources for it. Difference to unicast polling is that instead of reserving BR allocations for individual SS CIDs there exist allocations for multicast or broadcast CID in UL-MAP which SSs may use to request bandwidth. [10]

\section{MAC management messages}

The management messages are designed for controlling actions at SS, RS or BS on request/response/acknowledgment basis. Some of the events triggered or executed by the management message exchanges are the following:

- Network entry and registration.

- Handover.

- Privacy key exchange.

- Dynamic service addition, modification and removal.

- UL and DL physical channel description on DCD/UCD messages.

- Delivery of UL and DL user access information on DL-/UL-MAP messages. 
- Multicast polling.

Detailed information on these procedures can be found, for instance, in [1]. Later in this document especially network entry and handover are dealt with more accurately.

\section{ARQ}

Automatic Repeat Request (ARQ) is an error control mechanism used on a perconnection basis in which dropped or erroneous packets are retransmitted by the sender. ARQ works on WiMAX MAC layer and it can be used for retransmission of packed and fragmented MAC SDUs. If ARQ is enabled, the transmitter, after sending a PDU, waits for an acknowledgment from the receiver indicating it has received the packet. If not acknowledged or after a negative confirmation due to dropped or erroneous PDU, the transmitter resends the packet. An ARQ feedback message, containing acknowledgment information, can be sent as a separate management message or piggybacked within a packet payload. [8]

Depending on the traffic type ARQ might have a big positive impact on QoS. The effect of ARQ on VoIP is inspected in [9]. It shows that especially delay in voice application can be reduced by means of correct ARQ parameters.

\section{Scheduling and persistent scheduling}

Scheduling service is used for managing data transmissions across the air interface. It resides at BS (or RS) which controls the UL traffic by allocating users resources for their transmissions. Scheduling for each connection is determined by the QoS parameters exchanged between entities during addition or modification of a transport connection. QoS parameters include one of so called scheduling types, which are described later in QoS subsection. The scheduling type is chosen according to traffic type, such as VoIP or video, which changes the behavior of the scheduler. For instance, traffic for UGS connection is handled with greater priority than BE traffic. Also multicast and broadcast requests alter scheduler's actions when compared to unicast. [8]

BS controls also DL scheduling which is a bit more simple when compared to the UL scheduling. The BS fills bursts in DL subframe according to bandwidth needs or QoS parameters of SSs, and then downlink traffic is broadcasted to all SSs. [8]

The persistent scheduling mechanism reduces overhead with VoIP traffic by allocating persistently resources for VoIP connections for multiple frames ahead. Since 
SS does not need to request for resources in each frame, the MAP signaling decreases, thus less overhead is produced. [8]

\section{Contention resolution}

When multicast or broadcast polling is used, there might occur collisions in SSs' bandwidth requests since SSs may request bandwidth during any request interval allocated for the multicast or broadcast CID in the UL-MAP. To reduce collisions SSs that need bandwidth and take part in the polling run a contention resolution algorithm to select the slot for their BR IE in the UL-MAP. [8]

\section{Network entry}

One of the essential procedures in any wireless technology is network entry of subscriber and registration of user equipment to network. Figure 2.5 represents the initial network entry process which a SS has to go through in order to be able to operate in WiMAX network.

The process depicted in the figure is split into the following main phases in [8]:

1. Synchronization to the BS - The SS, when powered up or after loosing signal, tries to detect a DL channel by scanning downlink frequencies and listening to DL frame preambles. After detecting a DL-MAP the SS performs frequency and timing synchronization and decodes the DL-MAP for discovering the BS, Network Access Provider (NAP) and possibly Network Service Provider (NSP) information. [8][14]

2. Obtaining DL and UL parameters - After synchronization the SS listens for control messages, such as DCD, UCD, DL-MAP and UL-MAP, to receive information on the channel. According to received parameters, the SS decides whether the channel is suitable for it and either retains the linkage or scans for other channels. [8]

3. Initial ranging - If SC or OFDM PHY is used, the SS starts initial ranging by transmitting a ranging request (RNG-REQ) to the $\mathrm{BS}$ when the initial ranging transmission opportunity occurs. When OFDMA is in use, first a CDMA ranging code is sent in the first possible ranging slot by the SS before the RNGREQ. During the initial ranging the BS allocates basic and primary management CIDs for the SS which are used from here on for management message 


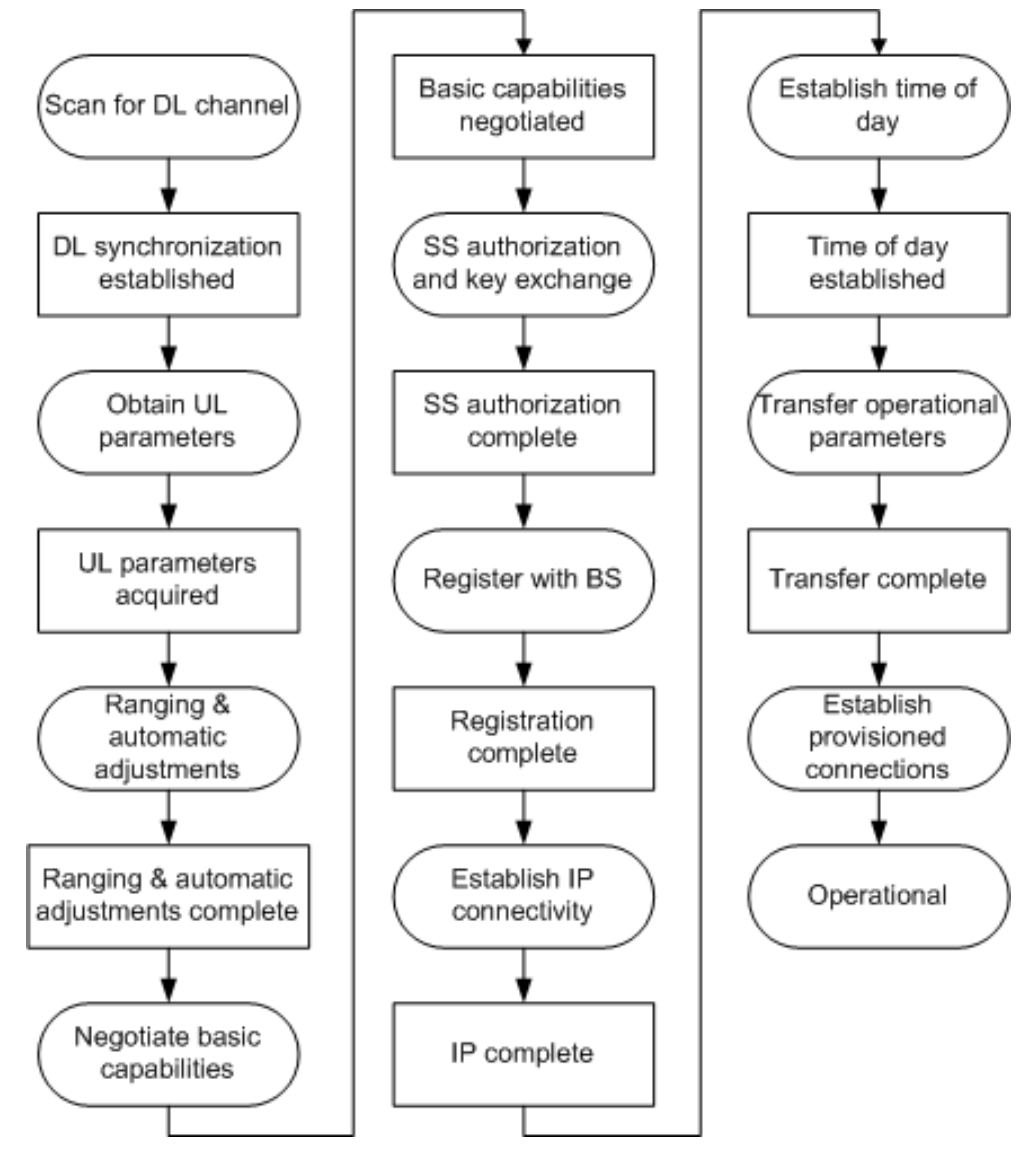

Figure 2.5: Network entry procedure.

transmissions and transmits this information to the SS within a ranging response (RNG-RSP) message. [8]

4. Negotiation of basic capabilities - After the initial ranging the SS sends a SBCREQ message to obtain basic capability information from the BS. The message includes MAC and PHY related queries for, e.g., bandwidth request and allocation purposes and number of HARQ channels. [8]

5. Authorization and key exchange - If PKM is enabled during the SBC exchange, the next step in network entry is authorization and privacy key exchange which are done via PKM-REQ and PKM-RSP messages. The PKMv2 authorization policy is used which may contain RSA- or EAP-based authorization and X.509 digital certificate utilization. [8]

6. Registration - The registration request (REG-REQ) message initiates the registration process during which the secondary management connection is created. One important piece of information carried by the REG-REQ is the du- 
ration of the Handover Indication Readiness timer. If a handover occurs and the serving BS transmits a MOB_BSHO-RSP/REQ, it will start this timer. After its expiration the BS may send an unsolicited UL grant to the SS for the MOB_HO-IND message. By this the SS will omit bandwidth requesting for the MOB_HO-IND and the handover process is expedited. [8]

7. Establish IP connectivity - Depending on the network layer implementation, fixed and mobile stations may use different methods for obtaining network address. Dynamic Host Configuration Protocol (DHCP or DHCPv6) mechanism with IPv4 and IPv6 or Stateless Address Autoconfiguration with IPv6 may be used for setting the IP address [12].

8. Establish time of day - The time of day information retrieval from the time server is defined in the IETF RFC 868. UDP is used for getting the time of day information which is needed at the SS and BS for time-stamping logged events. [8]

9. Transfer operational parameters - After obtaining the network address, the SS downloads a configuration file, which may contain, for instance, software server IP address and vendor-specific configuration settings. [8]

10. Set up provisioned connections - After successful transfer of the operational parameters the SS is ready for connection creation which is the last stage in the network entry process. The DSA-REQ, DSA-RSP and DSA-ACK messages are transmitted between the BS and the SS that provide entities enough information for creating the transport connections. One DSA transaction is performed for each new connection. During the message exchange the BS checks from the core network (AAA server at the CSN and anchor Service Flow Authorization (SFA) at the ASN-GW) whether a new service flow can be created for the user, and if so, the BS creates a new SFID which is used for mapping the service flow to the connection. [8][14]

\section{Ranging}

UL ranging consists of initial ranging explained in the previous section and periodic ranging. During the network re-entry the SS may perform also fast ranging to speed up the handover. After the initial ranging the SS continues with periodic ranging. It is used for adjusting transmission parameters during normal operation between the SS and the BS to maintain the UL communication. There are different definitions for 
periodic ranging for each PHY type in [8].

The fast ranging process exploits backhaul connections between BSs to accelerate the ranging process and network re-entry. If the fast ranging is enabled, the serving BS and the target BS may negotiate an unsolicited non-contention-based ranging opportunity for the MS in question, so the MS does not need to take part in the contention process to request for bandwidth for the transmission of the RNG-REQ. To be able to inform the MS about predefined ranging opportunity at the correct time, that is, when the MS will be ready to transmit the RNG-REQ, the target BS has to take into account the Handover Indication Readiness timer and the BS Switching timer. The first one was explained in the previous section. The BS Switching timer tells when the target BS should transmit an unsolicited UL grant to the MS for the RNG-REQ. [8]

\section{QoS}

The idea behind providing QoS in WiMAX lies on connection-oriented MAC architecture, service flow management and scheduling. Every time a SS and a BS need to communicate with each other, a unidirectional logical link, called uplink (UL) or downlink (DL) connection, is established. Each connection is mapped to a WiMAX service flow which works as a transport service for packets in UL or DL direction. The service flow defines QoS traffic parameters to be used for a connection. This include, for instance, traffic priority, maximum latency, tolerated jitter and maximum sustained traffic rate ensuring a specific level of service. Each service flow is identified by a 32-bit service flow identifier (SFID). [8][35]

Connections are controlled by a BS which is able to schedule traffic in DL and UL. The UL traffic control is done by means of bandwidth request/grant for allowing SS to transmit on predetermined time periods. The per-connection basis scheduling is useful for managing different traffic types, such as real time video or VoIP, FTP or Web browsing. Furthermore, the IEEE 802.16 specifies five scheduling types used with different kinds of applications. The following scheduling types are applied to service flows, affecting the usage of UL bandwidth request opportunities. [4][8][35]

- UGS - the Unsolicited Grant Service grants fixed-size UL allocations for an application minimizing the need for bandwidth requests, thus eliminating overhead and latency. This allows usage of real-time applications like VoIP.

- rtPS - the real-time Polling Service offers support for real-time UL transport with variable-size data packets. This makes it useful for video transmission. The rtPS optimizes data transport efficiency with a cost of slight overhead. 
- ertPS - similarly to the UGS, in the extended real-time Polling Service the BS provides unsolicited unicast grants for the SS. Unlike the UGS, the ertPS allows dynamic-size UL allocations which makes it good for real-time voice and video applications.

- nrtPS - using the non-real-time Polling Service offers user unicast BW request opportunities on a regular basis, making it applicable even when the network resources are limited due to congestion.

- BE - the Best Effort service type allows SSs to use contention request opportunities. It is designed to be used when there is no minimum requirements for the connection. This service type suits for Web browsing.

For each subscriber in the WiMAX network a QoS profile is defined and stored in the AAA server. This forms a basis for connection-oriented service, that is, associations between connections and service flows. The QoS profile stores allowable number of service flows, their scheduling types and values for other QoS parameters. [14][15]

Depending on the QoS profile and network properties the subscriber may use service flows provisioned via the network management system or dynamically created service flows. The provisioned service flows, including the initial service flow (ISF), can be created during the registration phase of the network entry after a successful authentication of the user. At this stage ASN-GW obtains SS's QoS profile from AAA server which it uses to initialize service flow creation. Provisioned service flows can be activated or deactivated at any time when the SS is connected to the network (applicable to both static and dynamic service models described below). The dynamic service flow creation may be requested/initiated by the SS or by the network whenever a new connection is needed. DSA, DSC or DSD message exchange is used for service flow creation, modification or deletion, respectively. $[8][15]$

There are two types of service models for subscribers, which affect the usage, addition, modification and deletion of service flows, namely static service model and dynamic service model. A subscriber is engaged to either of them depending on the authorization policy. Under the static service model the SS is neither allowed to change parameters of provisioned service flows nor create new service flows dynamically. The provisioned SFs established by service flow management (SFM) and service flow authorization (SFA) logical entities within an ASN-GW during the network entry are maintained as long as the SS is connected to network. The dynamic 
service model allows the SS or the BS to create, modify or delete dynamic service flows. With the help of Policy and Charging Control (PCC) framework this allows, for example, interworking between WiMAX and 3GPP/3GPP2/TISPAN IP Multimedia Subsystem (IMS), where IMS applications may dynamically request authorization for new service flows with volatile QoS requirements. [8][13][15]

\section{Power management}

The Mobile WiMAX standard defines two power saving methods for the mobile equipment: idle mode and sleep mode. Both rely on turning off components that are not needed at particular time. Usually the device is switched off when it does not transmit or receive data. The power saving modes may be enabled and disabled by management message signaling between the MS and the BS. [1]

In the sleep mode the mobile station turns off its devices for predetermined periods which are known also by the BS. After the unavailability period the MS changes into listening period in which it is able to transmit and receive data. The listening period is again followed by a sleep period. The standard defines three power saving classes. In the Power Saving Class 1 the sleep period is exponentially increased if no data traffic is perceived. The Power Saving Class 2 has fixed sleep and listening period lengths. The Power Saving Class 3 has only single sleep period which is usable with multicast or management connections. [1]

Idle mode conserves power and radio resources by completely turning off equipment that is not needed. Support for mobility during enabled idle mode is implemented better than with sleep mode. When the idle mode is enabled, the MS is assigned to a paging group, a cluster of base stations covering a large geographical region within which the MS is able to move without any UL signaling. During the active idle mode state the MS can be paged if there is DL traffic destined to it. [1]

\section{Handover and mobility}

The MAC layer provides methods for controlled transition of MS between different BSs without significant loss of data or decreased QoS. The handover mechanisms and mobility support in WiMAX are described in detail in section 2.10.

\section{Multicast and broadcast services}

Multicast and broadcast are used to deliver messages to a group of clients. In WiMAX the multicast and broadcast services (MBS) are supported with a set of fea- 
tures, which include signaling messages to establish MBS, MBS associated QoS and encryption management and support for macro diversity for improved MBS traffic delivery. [4]

\subsubsection{MAC security sublayer}

Creating a secure wireless channel is demanding when compared to securing wired part of the network. It is, for example, difficult to detect undesired eavesdropping or altered data at the receiving side of the air interface. The security scheme can be observed from the user point of view and also from the service provider's perspective. For users it is important to preserve privacy and data integrity, whereas the service providers aim for preventing unauthorized use of the network. [4]

The MAC security sublayer along with the core network operations is responsible for securing data transmissions. Privacy support is set up from two component protocols. The first is an encapsulation protocol that provides security for data packets over fixed BWA network. This protocol defines pairings of data encryption and authentication algorithms and rules for applying them on MAC PDUs. The second component protocol is a key management protocol (PKM) which is used for secure distribution of keying data from BS to SS. Data encryption is performed at WiMAX MAC layer which uses Advanced Encryption Standard (AES) to create the cipher data. HMAC SHA-1 is used for ensuring data integrity. The Mobile WiMAX adopts Privacy and Key Management Protocol version 2 (PKMv2) which takes care of key exchange using RSA and authentication using Extensible Authentication Protocol (EAP). [1][8]

\subsection{WiMAX Forum}

So far an overview of the IEEE 802.16 standard and aspects affecting the wireless link between nodes have been introduced. Rest of the section 2 is devoted to access network segment of the WiMAX technology and mobility support. The access network functionality which is mostly defined by WiMAX Forum.

WiMAX Forum is a non-for-profit organization consisting of several equipment manufacturers and network providers. It was founded in 2001 to specify interoperability of WiMAX based equipment to IEEE 802.16, ETSI HiperMAN and WiBro/ Mobile WiMAX standards. One of the main tasks of WiMAX Forum is to organize working groups to deal with essential issues in deploying WiMAX Forum certified products to market. Member list and other information on WiMAX Forum can be 
found in [7].

\subsection{Network Working Group (NWG)}

The 802.16 standards define air interface operations in WiMAX network. In addition to wireless specification also definition of the backhaul and backbone functions and network elements is mandatory for fully operational telecommunication technology [7]. The Network Working Group (NWG) constituted by the WiMAX Forum is responsible of promoting WiMAX beyond IEEE's 802.16 specification [7]. Later in this document some of NWG's outcomes are more closely presented and also utilized during the simulations.

\subsection{Network reference model}

The Network Reference Model (NRM) is a logical depiction of the WiMAX network architecture. As seen from figure 2.6, the network architecture consists of functional entities and reference points which allow interoperability between the entities. The reference points bind together two different entities and introduce protocols for function interoperability [14]. In the figure solid line represents control plane which allows network management information conduction. Dashed line represents bearer plane which is responsible for data payload transportation. The reference points R2, R3 and R5 are outside of the scope of this thesis.

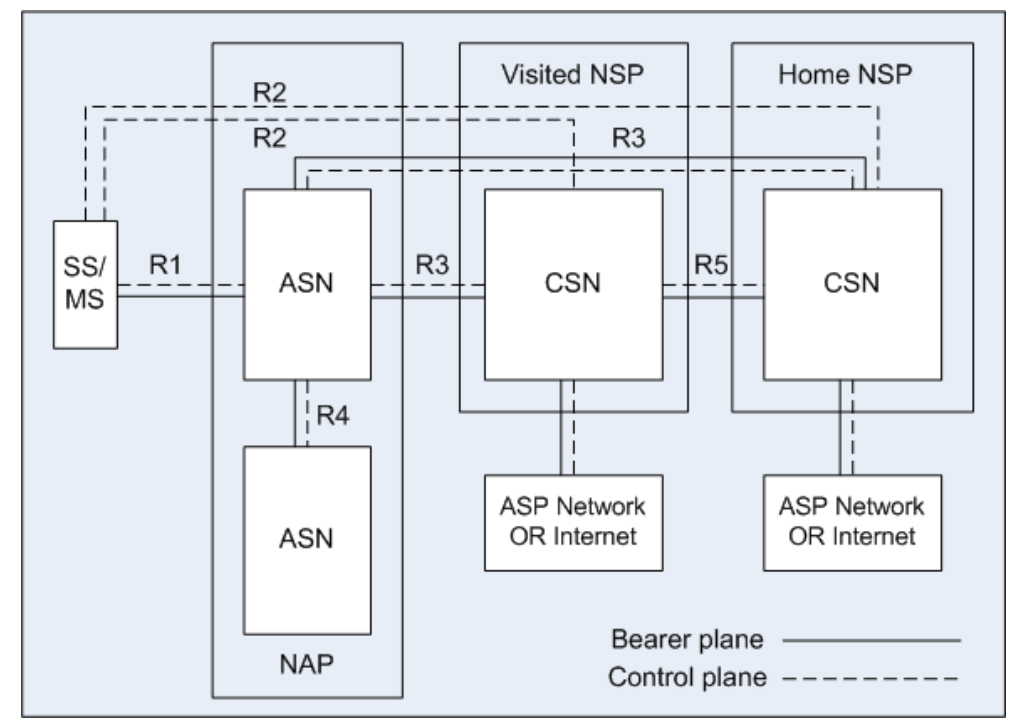

Figure 2.6: Network reference model. 
The reference point R1 provides a mobile node a wireless access to WiMAX network. The R2 between the mobile node and the Connectivity Service Network (CSN) is a logical reference point operated by the home or the visited NSP and it is used for user authentication and authorization and IP Host Configuration management. The R3 includes control and bearer plane protocols between the ASN and the CSN providing user data transfer, AAA, policy enforcement and mobility management capabilities. The reference point $\mathrm{R} 4$ exposes control and bearer plane protocols between different ASNs. It allows coordination of the mobile node movement between similar or heterogeneous ASNs. The R5 introduces connectivity and interoperability between the home NSP and the visited NSP. [14][15]

\subsection{Network elements}

\subsubsection{Subscriber Station (SS)}

A customer premises equipment (CPE) in WiMAX is called a subscriber station (SS) or a mobile station (MS, a term used for mobile user terminals), which is used to connect the user to wireless network. It contains PHY and MAC layer functions and necessary physical equipment for fixed and/or mobile communication. [1][4]

There exist different types of fixed position terminal equipment for various purposes which are generally divided into indoor-CPEs and outdoor-CPEs. In addition to these WiMAX support can be applied to smaller mobile devices. Some examples are shown in figure 2.7. [4]

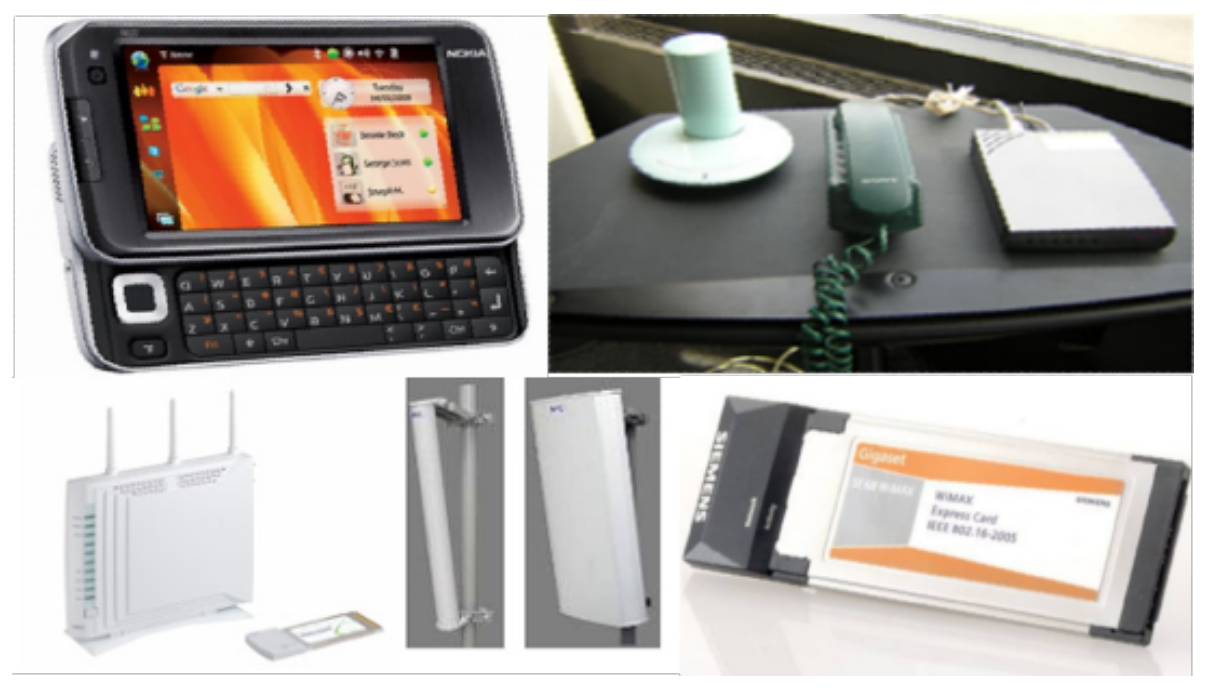

Figure 2.7: Examples of subscriber stations. 
When operating in high frequencies obstacles like brick walls, concrete walls or even glass windows may have a big impact on received signal level. Therefore, fixed outdoor-CPEs usually provide better signal level due to less deterioration by walls or roofs, and in best case offers a LOS condition to the BS or the RS. Downsides of outdoor-CPEs are their expensiveness and complexity of installation which are not so big issues with indoor-CPEs. [4]

\subsubsection{Access Service Network (ASN)}

The Access Service Network is a logical entity that provides network access services for subscriber stations. Logical composition of an ASN containing a single ASN gateway (ASN-GW) is depicted in figure 2.8.

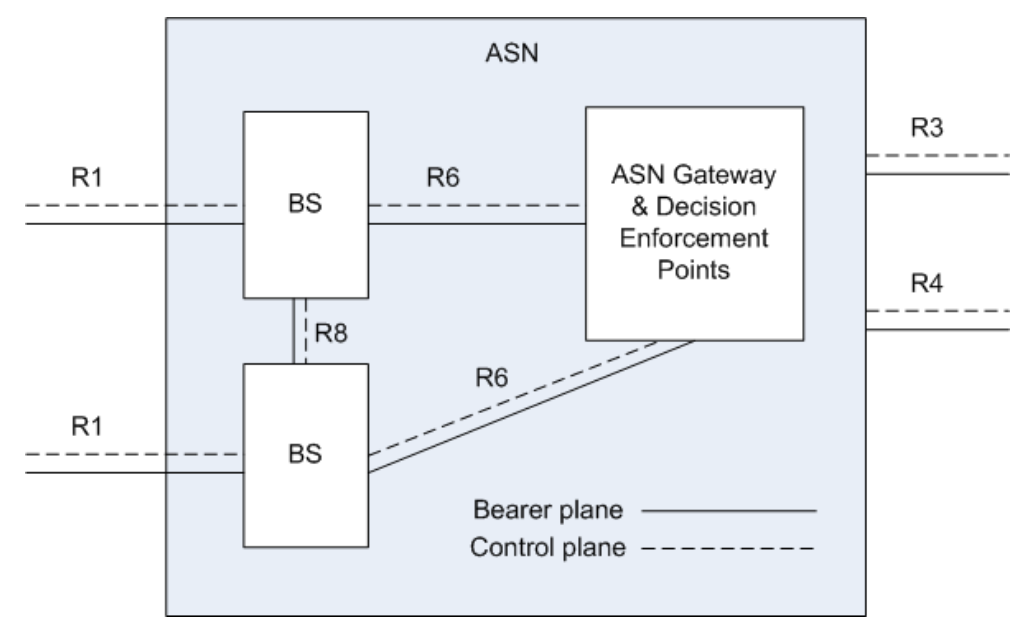

Figure 2.8: ASN-GW reference model.

One ASN comprises at least one BS and one ASN-GW connected by R6 reference point. Multiple BSs within one ASN are connected via R8. If there is more than one ASN-GW in an ASN, they are connected via R4 as are the ASN-GWs within different ASNs. User mobility within one ASN is called Intra-ASN mobility and mobility between ASNs without R3 signaling is called Inter-ASN mobility. [14][15]

\section{ASN Gateway}

ASN-GW is a logical traffic and control plane aggregation point within an ASN. It may perform bearer plane routing, bridging, redundancy and load-balancing control among other ASN-GWs. Here are some of the mandatory tasks performed by the ASN-GW [13]: 
- Data path management functionality.

- Proxy-Mobile IP (PMIP) client (if PMIP is used).

- AAA client.

- Paging controller.

- Location register with ASN.

- Authenticator/Key Distributor.

- DHCP Proxy/Relay.

- Service flow management.

The ASN-GW can be logically decomposed into Decision Point (DP) for control plane functions and Enforcement Point (EP) for bearer plane functions, as depicted in figure 2.9. If the division is done this way, the DP and EP are separated by the Reference Point R7. If not decomposed, the R7 will not be utilized. [14]

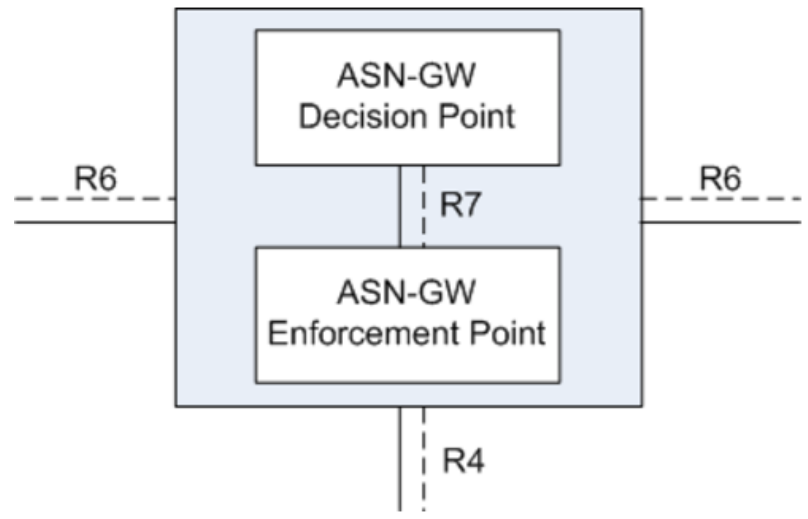

Figure 2.9: ASN-GW decomposition.

A subscriber station associates with one ASN-GW at a time. When entering the network the SS is assigned an anchor ASN-GW which is the traffic transit point between the SS and the IP core network. [14]

\section{Base Station}

WiMAX BS is a basic logical network entity that provides network access for user equipment. It implements WiMAX PHY and MAC layer specification. A logical instance of a BS may contain one or more physical BSs which are usually deployed to 
provide sector-based coverage. A base station is capable of scheduling and providing QoS for users allowed to operate in the network, whereas subscriber information dependent tasks are accomplished elsewhere, at the ASN-GW or the CSN. [8][14]

The IEEE 802.16j standard extends the WiMAX BS specification by introducing the Multihop Relay BS (MR-BS) for the relay networks. The MR-BS is fully compliant with 802.16e standard. The MR-BS is able to control the connectivity to both the relay station and the subscriber station. [3]

\section{Relay Station}

In Multihop Relay (MR) networks traffic and signaling between the SS and Multihop Relay BS (MR-BS) may be relayed by one or several Relay Stations (RS). Usually connections between RSs and other access stations are carried out over LOS wireless channel. MSs may have also NLOS connections to RSs. There are two types of RSs: transparent RSs and non-transparent RSs, usage of which differs from each other a bit. Transparent RSs are bridging links between MSs and MR-BSs. They do not take part in management signaling but just forward traffic and control messages between stations. Transparent RSs mostly provide extended coverage, whereas nontransparent RSs can also increase network capacity. A non-transparent RS transmits DL frame-start preambles, FCHs, MAP messages and DCD/UCD messages. This allows usage of distributed scheduling in which the bandwidth allocations of RS's subordinate stations are determined by both the RS and the MR-BS. A transparent RS is able to operate only in centralized scheduling mode in which BW requests are handled at the MR-BS. [3]

Figure 2.10 demonstrates usage of RSs. It depicts how the user can connect to either the MR-BS or the RS. Supporting relay stations bring a whole new challenge to network's resource and mobility control. Applying Relay Stations introduces new handover scenarios where the MS should be able to move from coverage area of the MR-BS to coverage area of the RS, and vice versa, or between RSs without significant delays or data loss.

The IEEE 802.16j also introduces support for RS's movement. These Mobile Relay Stations (MRS) are able to migrate from the air-interface of one access station (MR-BS or RS) to the air-interface of another access station [3]. The handover process for a MRS is performed similarly to handover of a MS. MRSs and handovers in relay networks are left outside the scope of this thesis. 


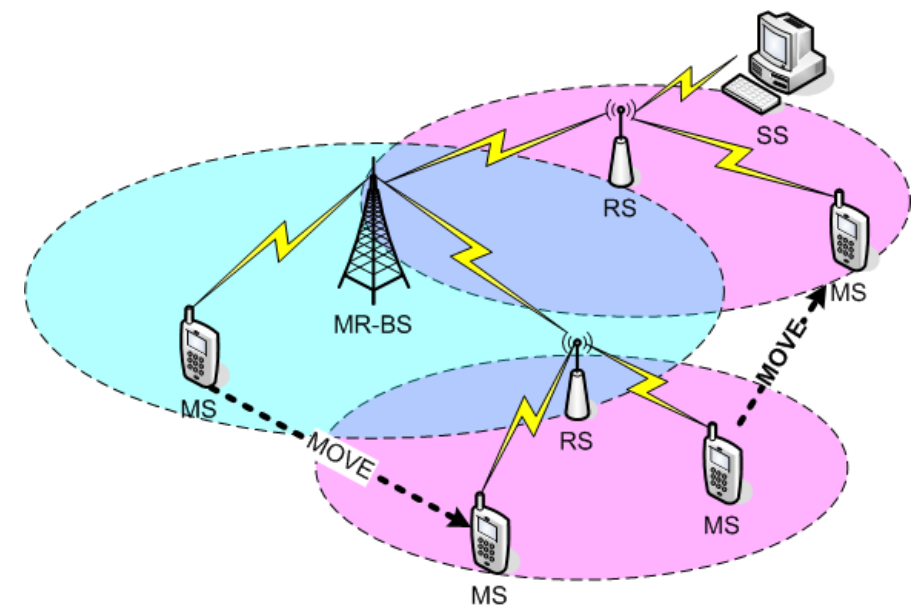

Figure 2.10: Usage of Relay Stations.

\section{ASN profiles}

The NWG specification [14] defines three profiles for the ASN that map ASN functions to ASN-GW and BS in different manner. Each of the profiles can be implemented by vendors and once a profile is chosen, implementing other profiles is not mandatory. Neither all the functions of a profile have to be implemented; the profile definition just tells where the existing functions are located. Although the profile implementations vary, they should be compatible with each other in case of intervendor operations.

\section{Profile A}

Some of the features of the profile A includes the following [14]:

- Handover control is located in the ASN-GW.

- Radio resource controller (RRC) is in ASN-GW which allows radio resource management (RRM) among multiple BSs.

- ASN-anchored mobility among BSs shall be achieved by exploiting R4 and R6 physical connections.

Figure 2.11 shows the locations of functions and reference point associations used for intra-ASN operations, namely operations between ASN-GW and BS located in the same ASN. 


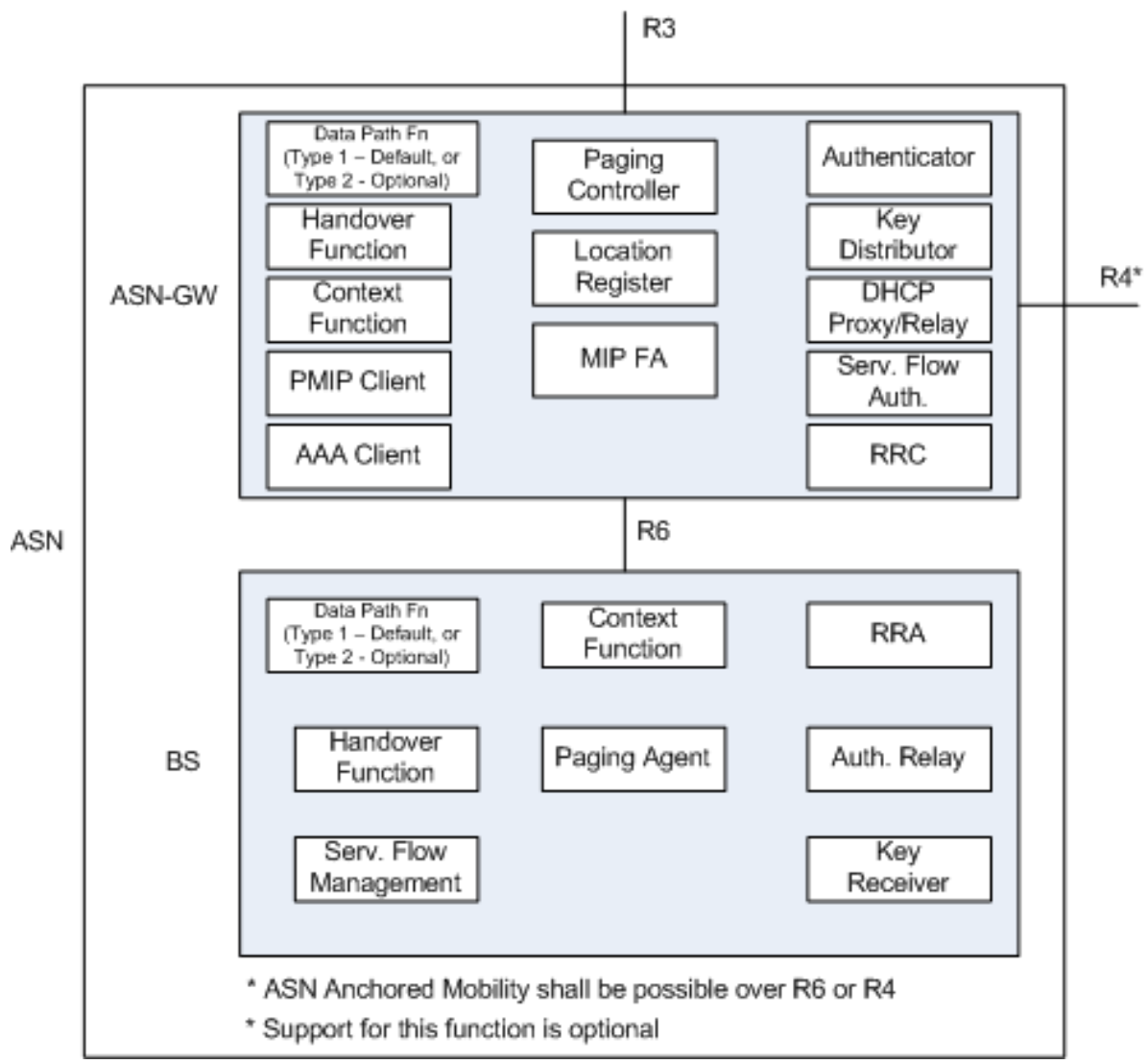

Figure 2.11: ASN profile A.

\section{Profile B}

The profile B ASN does not define intra-ASN interfaces which can be seen from figure 2.12. This profile allows flexible realization of ASN function mapping from single physical device containing all the functions to distribution of functions over multiple network nodes. The profile B ASN interoperates with other ASNs over R3 and R4 reference points. Inter-ASN anchored mobility works on R4. [14][15]

\section{Profile C}

Some of the main characteristics of this profile are the following [14]:

- Handover control is located in the BS

- RRC is in the BS that would allow RRM within the BS. A 'RRC relay' is located in the ASN-GW to relay the RRM messages between base stations via R6 reference point. 


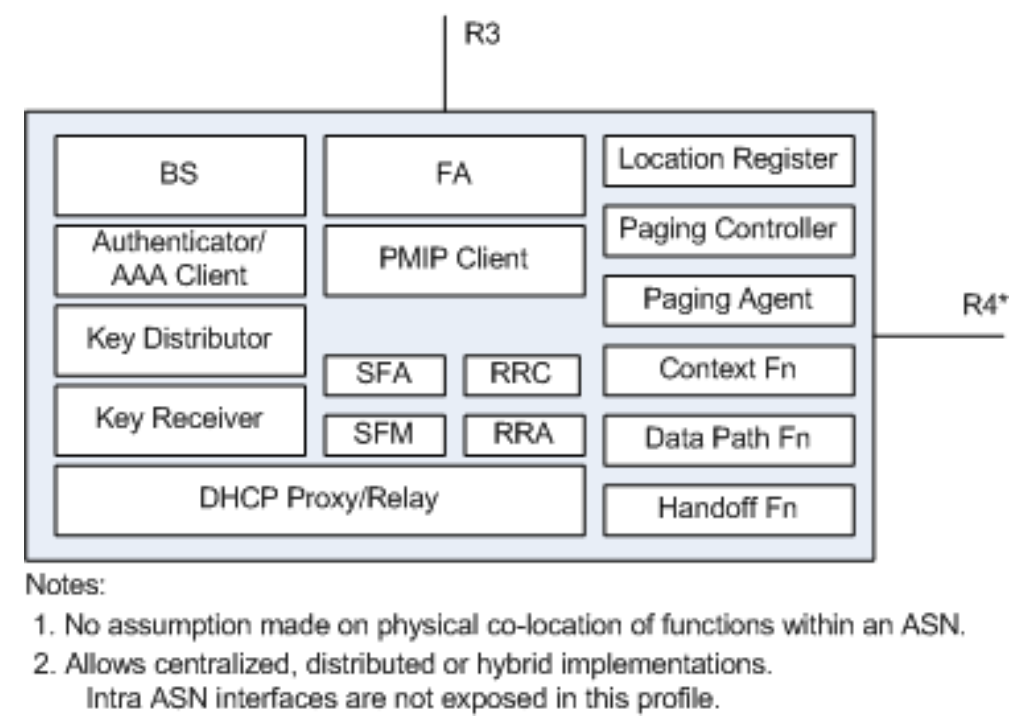

Figure 2.12: ASN profile B.

- ASN anchored mobility among BSs shall be achieved by utilizing R6 and R4 physical connections, as in the profile A.

Unlike the profile $\mathrm{B}$, the profile $\mathrm{C}$ divides the ASN functions into an ASN-GW and a BS as depicted in figure 2.13.

\subsubsection{Connectivity Service Network (CSN)}

The Connectivity Service Network is maintained by the ASP and it acts as a gateway between WiMAX ASN and public or other external networks. It provides authentication, authorization and accounting (AAA), mobility management between CSNs and IP services for WiMAX users. [14]

The CSN deployment enables mobility between different CSNs which is called CSN-anchored mobility, containing intra- and inter-NAP mobility over R3 reference point. It also may allow inter-technology handovers. [14]

\subsection{Mobility in WiMAX}

The principle of allowing the end-users to move and change the point of attachment (PoA) while using wireless network services is a distinct feature of the developed telecommunication technologies. Providing reliable and sufficiently fast data connections for fixed and nomadic subscribers across the air interface has been a minimum requirement for wireless technologies ever since the $3 \mathrm{G}$ networks emerged. 


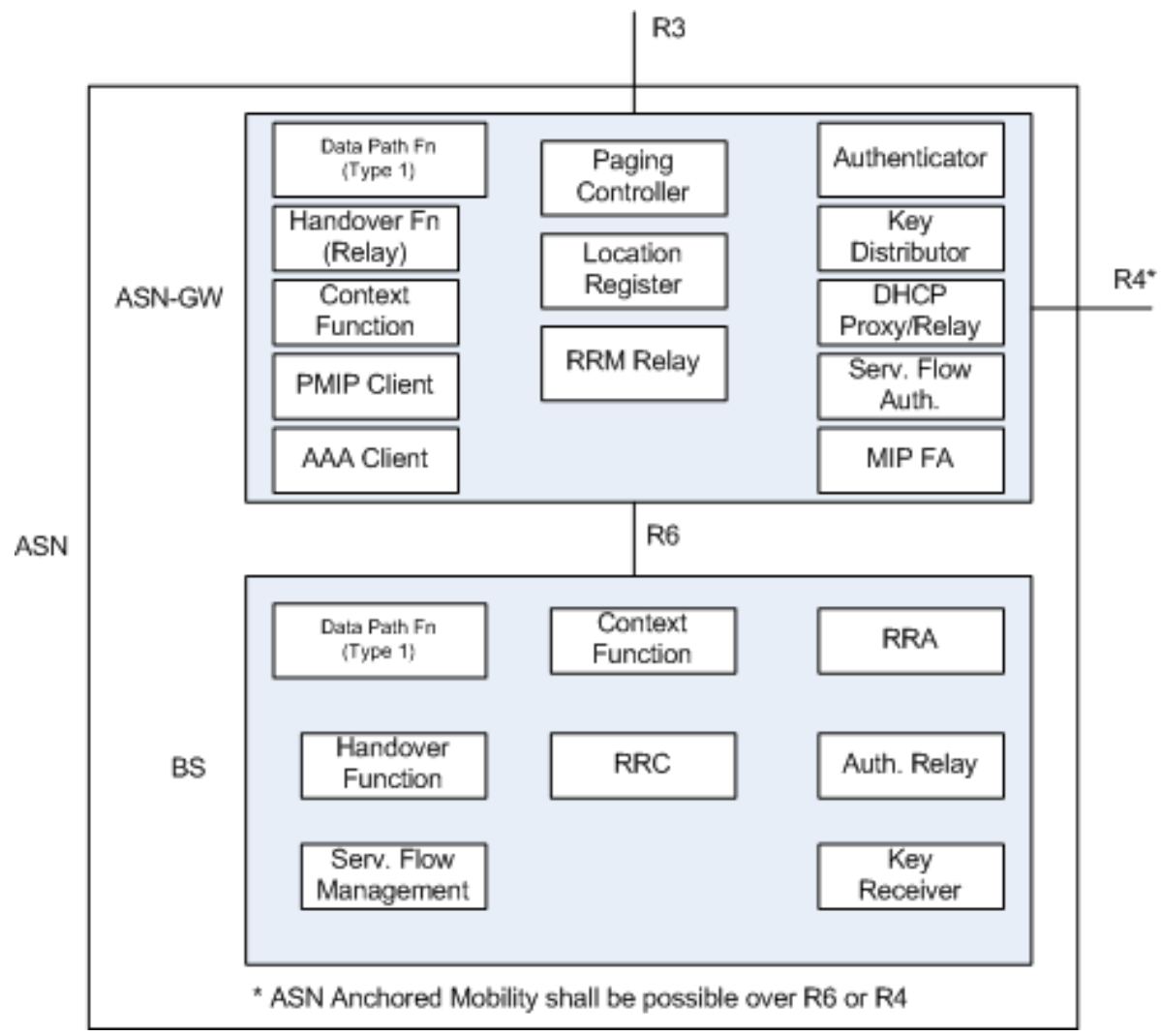

Figure 2.13: ASN profile C.

Current technical and infrastructural status favors development of high speed BWA systems. On the one hand, now clients are enticed by small, portable equipment allowing them to get online in spite of the location and still have full mobility support. On the other hand, now that dial-up based wired network access methods are vanishing and are not replaced everywhere by newly distributed fiber-optic links, wireless access may be the only way people can connect themselves to the Internet.

In [8] the handover process from the air interface perspective is divided into five sections: cell reselection, handover decision and initiation point, synchronization to new BS, ranging process with the new BS and context termination at the old BS. In addition to these, handover cancellation procedure is a mandatory feature quantified by the standard. Furthermore, actions in the access network between a BS, ASN and CSN are defined in detail. These topics are reviewed in this section.

Exploring the handover performance should not confine to mere air interface investigation since many operations are performed inside the access and core network. This thesis concentrates on handovers from wide perspective by exploring ASN-anchored mobility. The NWG also defines CSN-anchored mobility but the simulator extension used in this thesis does not support it. Thus, it is not observer 
in the thesis. The following subsections provide knowledge on handover types presented by the IEEE 802.16 , handover process and mobility types.

\subsubsection{Handover types}

Usually a handover is understood as a change of physical connection point through which the terminal communicates with network services. In WiMAX this is called inter-cell handover. There exists also so called intra-cell handover which basically means changing from one frequency to another while the serving BS remains the same. This feature could be exploited for example in a femto cell scenario where a user moves from outdoors to indoors. From wide perspective handovers may be split into two groups: horizontal $\mathrm{HO}$ and vertical $\mathrm{HO}$. In the horizontal $\mathrm{HO}$ network technology remains the same, whereas the latter is an inter-technology HO type. Media Independent Handover (MIH) is a vertical HO method defined in 802.21 specification [22]. In this thesis only inter-cell horizontal handovers are examined closely.

There are different handover triggering ways. One natural method is based on signal level measurements. If the received signal level from the serving BS is deteriorated enough, resulting in better signal quality from one or more neighboring BSs, then it is reasonable to perform a HO. This method is also used in the simulations later in this document. Depending on the network characteristics and properties there might exist several other reasons for HO initiation, including lack of BS's capacity, interference from other cells, fluctuating MS conditions and another network type offering a better performance. [21]

As mentioned before the WiMAX specification defines three types of handovers: hard $\mathrm{HO}(\mathrm{HHO}), \mathrm{FBSS}$ and $\mathrm{MDHO}$. From these, only implementation of the $\mathrm{HHO}$ is mandatory. The FBSS and the MDHO are optional. [8]

\section{Hard handover}

Hard handover is an efficient yet simple mechanism that is based on received signal strength indication (RSSI) measurements and support for it is mandatory in mobile WiMAX network. Hard handover is a break-before-make BS switching method where the MS first stops connectivity with the old BS before engaging to the target one. The $\mathrm{HHO}$ is bandwidth efficient but produces longer delays than MDHO and FBSS which are considered as soft HO mechanisms. [14][18]

To improve HHO performance and to keep MS's unavailability durations small, many optimization methods are introduced in the specification. A MS initiated 
$\mathrm{HHO}$ process presented in section 2.10.2 is an optimized $\mathrm{HO}$ method in which the backhaul network is exploited in the $\mathrm{HO}$ preparation and action phases.

\section{MDHO and FBSS}

Macro Diversity Handover and Fast Base Station Switching are considered as soft handover methods. They both utilize a so called 'diversity set' which is a list of BSs included in the handover. These BSs in the diversity set are located near the MS. BSs that are located within the communication range of the MS but are providing low signal strength level are not included in the diversity set, but are defined as neighbor BSs.

In MDHO the MS is able to communicate in DL and UL with all the BSs in the diversity set simultaneously instead of only one. In DL diversity combining and in UL selection diversity are used for improving quality of the received signal. [8][20]

In FBSS the MS communicates only with one anchor BS over UL and DL channel. Switching the anchor BS is quite easy due to MS's continuous monitoring of other BSs in the diversity set. The anchor BS changing process can be performed either by using $\mathrm{HO}$ messages or by using fast anchor BS selection feedback over the fast feedback channel. [8][20]

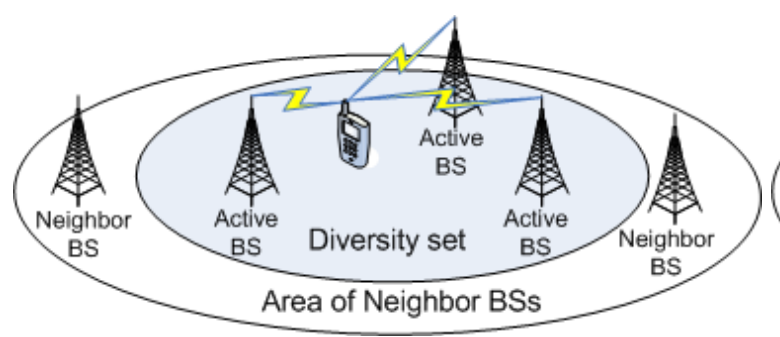

(a) Macro diversity handover.

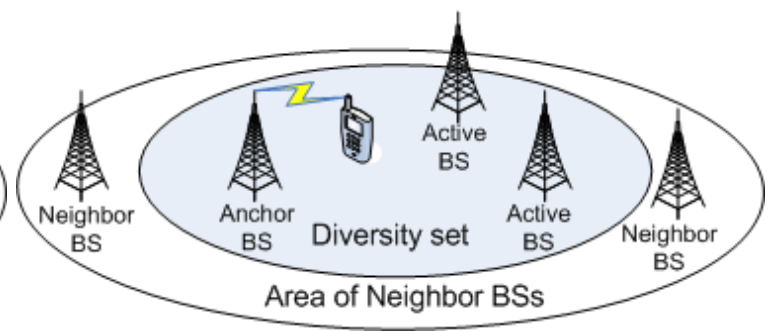

(b) Fast base station switching.

Figure 2.14: Soft handover mechanisms.

In MDHO and FBSS the diversity set has to be updated from time to time. A BS is removed from the diversity set if its long-term CINR drops below a preset threshold. Equivalently, a neighbor BS may be added to the diversity set if the long-term CINR is high enough. The diversity set update procedure is commenced by the MS or the (anchor) BS with a transmission of a MOB_MSHO-REQ or a MOB_BSHO-REQ message, respectively. [8][20] 


\section{Media Independent Handover (MIH)}

The Media Independent Handover is defined in IEEE 802.21 which is a standard for handover services between heterogeneous network types. WiMAX is supported by the IEEE 802.21 among other IEEE family standards and media types defined by 3GPP and 3GPP2. The IEEE 802.21 standard defines link-layer functionality but does not provide definitions for intra-technology handovers, handover policies, security mechanisms or handover enhancements for layer 3 and above. [22]

\subsubsection{Handover process}

During normal operation the MS continuously surveys signal level from the serving BS. To receive signal strength information from neighbor BSs it may decode periodical neighbor advertisement messages (MOB_NBR-ADV) sent by the serving BS or it may transmit a request to the serving BS to schedule a scanning interval for the MS’s neighbor BS RSSI measurements.

If periodical transmission of the neighbor advertisement messages is enabled, the serving BS includes suitable neighbor BS information in a MOB_NBR-ADV, which it sends to the MS. This will facilitate scanning process because the MS then knows which neighbor BSs it should prefer while seeking a target BS. The serving BS uses backbone network to gather information on the neighboring BSs. [8][16][17]

When the MS utilizes scanning of neighbor BSs, it may decide to transmit a scanning interval request message (MOB_SCN-REQ) to the serving BS if the handover trigger conditions defined in the DCD message are met. The serving BS may then grant a scanning interval allocation encoded in MOB_SCN-RSP management message. Scanning is used for distinguishing suitable target BSs for the handover. During the scanning intervals the MS is able to create basic association with the neighbor BSs for synchronization and registration to mitigate signal quality measurements. During the scanning phase user data is buffered but not transmitted in UL or DL. This phase of the handover produces quite long packet delays and optimization of which has been of interest to many studies. [16][17][18]

As specified in [8], the MS may define one of the following scanning types in the MOB_SCN-REQ message which it uses for requesting initial ranging parameters and service availability information from neighboring BS. Performing association is not mandatory for a mobile station. [8]

- Scanning with association level 0: The serving BS allocates periodical contention based ranging allocations for the MS to perform ranging with neigh- 
boring BS but the neighboring BSs have no knowledge on the MS.

- Scanning with association level 1: The MS transmits a MOB_SCN-REQ to the serving BS with a list of neighbor BSs it wishes to associate with. Then each of these BSs provide a predefined ranging interval to the MS.

- Scanning with association level 2: This is a network assisted association method pretty similar to association level 1. In this type the neighbor BSs do not have to transmit RNG-RSP messages since the information on the PHY offsets is sent over the backbone network between the neighbor BSs and the serving BS. The serving BS transmits all ranging related information in a MOB_ASC_REPORT to the MS.

The handover process can be initiated by the mobile itself or it can be network initiated. When a decision to migrate the MS from one BS to another is made, the first action in the handover preparation is transmission of a handover request message; in mobile initiated handover a MOB_MSHO-REQ message is transmitted by the MS and in network initiated HO a MOB_BSHO-REQ is sent by the BS. A handover request message contains information on pending handover type (hard $\mathrm{HO}$, FBSS or MDHO) and identifiers of suitable target BSs. When the MOB_MSHO-REQ is received the serving ASN transmits a HO_Req on R4 to each target ASN. This message contains especially MS's service flow information needed for re-establishment of the connections after the HO. When the target ASN receives a HO_Req message it may retrieve the Authorization Key (AK) context from the authenticator ASN if it is not included in the message. Furthermore, at this stage data path may be preestablished between the target ASN and the anchor ASN. [8][14]

Upon processing the $\mathrm{HO} \_$Req the target ASN transmits a $\mathrm{HO}$ _Rsp message on $\mathrm{R} 4$ reference point containing possible information on data path pre-registration. When the serving BS notices the HO_Rsp it performs a transmission of the MOB_BSHO-RSP to the MS over the air interface and it also sends an acknowledgment message to all candidate BSs over the R4 to complete the handover preparation. The purpose of the MOB_BSHO-RSP is to inform the MS on BSs it may attach itself during the actual handover. The message may also contain information on preallocated fast ranging time interval that will boost the network re-entry after the HO. [8][14]

The MS determines the final target BS and includes this information to a MOB_HO-IND message before transmitting it to the serving BS. The handover indication message is the last message to the serving BS before the actual HO. After its transmission the MS removes old connections and initiates ranging with the target BS. 
Upon reception of the MOB_HO-IND the serving BS will transmit a HO_Cnf message to the target ASN over the backbone network to finalize the $\mathrm{HO}$ from its part. The target ASN then replies with a HO_Ack message. [8][14][15]

After the MS has performed a network re-entry with the target BS, a data path registration has to be carried out between the target ASN and the anchor ASN for bearer plane procedures. If the optional data path pre-establishment was done before then this stage requires only confirmation of the data path registration. Otherwise a full registration procedure has to be performed using Path_Reg_Req, Path_Reg_Rsp and Path_Reg_Ack messages. When the path registration exchange is completed a HO_Complete message is transmitted from the target ASN to the serving ASN to inform about completion of the handover. Figure 2.15 depicts a successful, MS initiated HO process and message exchanges. Note that the context retrieval procedure and the data path pre-registration procedure may be performed at various points during a HO. [8][15]

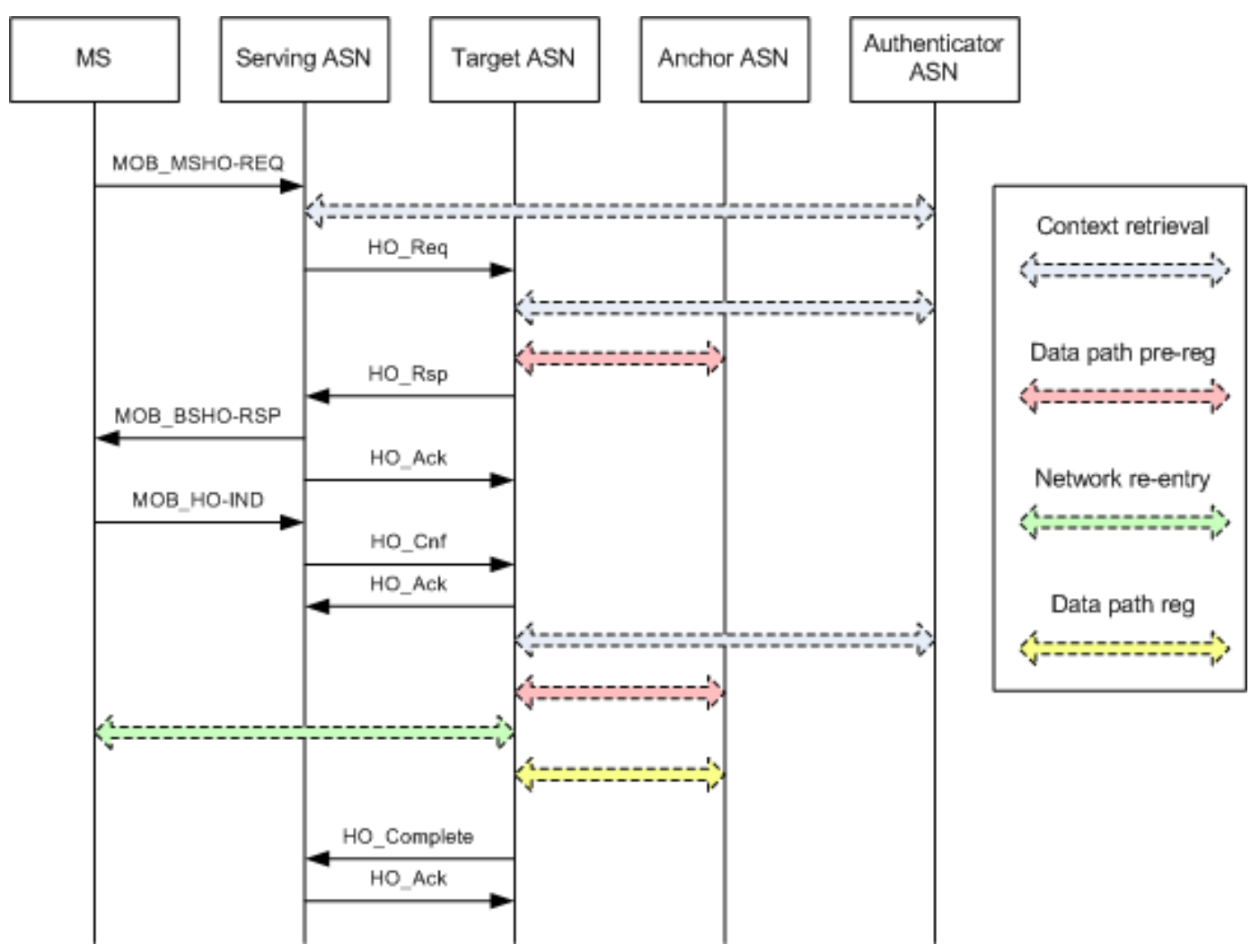

Figure 2.15: A successful handover process.

The network entry after a $\mathrm{HO}$ differs from the initial network entry explained in section 2.5.2. During the handover message exchanges parts of the MS's context may have been transmitted to the target ASN, which at this stage can be omitted. If 
the target BS did not receive the context it may request it from the serving BS over the backbone network. This sort of optimized HHO is designed to keep the handover interruption times less than $50 \mathrm{~ms}$ to make usage of VoIP and other real-time applications possible. When an optimized handover is performed, it is possible to speed up the process by leaving out some of the network entry signaling. This includes basic capability negotiation, parts of PKM authentication, registration messages and service flow encodings. [8][14]

\subsubsection{ASN-anchored mobility}

In the ASN-anchored mobility a MS is allowed to move across coverage areas of BSs without changing Foreign Agent (FA) and Care-of-Address (CoA) points. In other words, in the ASN-anchored mobility the CSN and the anchor ASN used for communication do not change. The ASN-anchored mobility can be divided into intra-ASN mobility and inter-ASN mobility. In the first type a MS may transit across different cells within the same ASN, whereas in the latter the serving ASN can change. The ASN-anchored mobility management (MM) covers signaling between the ASNs and the BSs over the R4 and the R6 reference points and packet loss, order and latency control. The ASN-anchored mobility is logically composed of a Data Path (Bearer plane) function, a Handover function and a Context function (figure 2.16) which are responsible of data transmissions and control message exchanges. These functions are attached to different network entities depending on their tasks. [14][15]

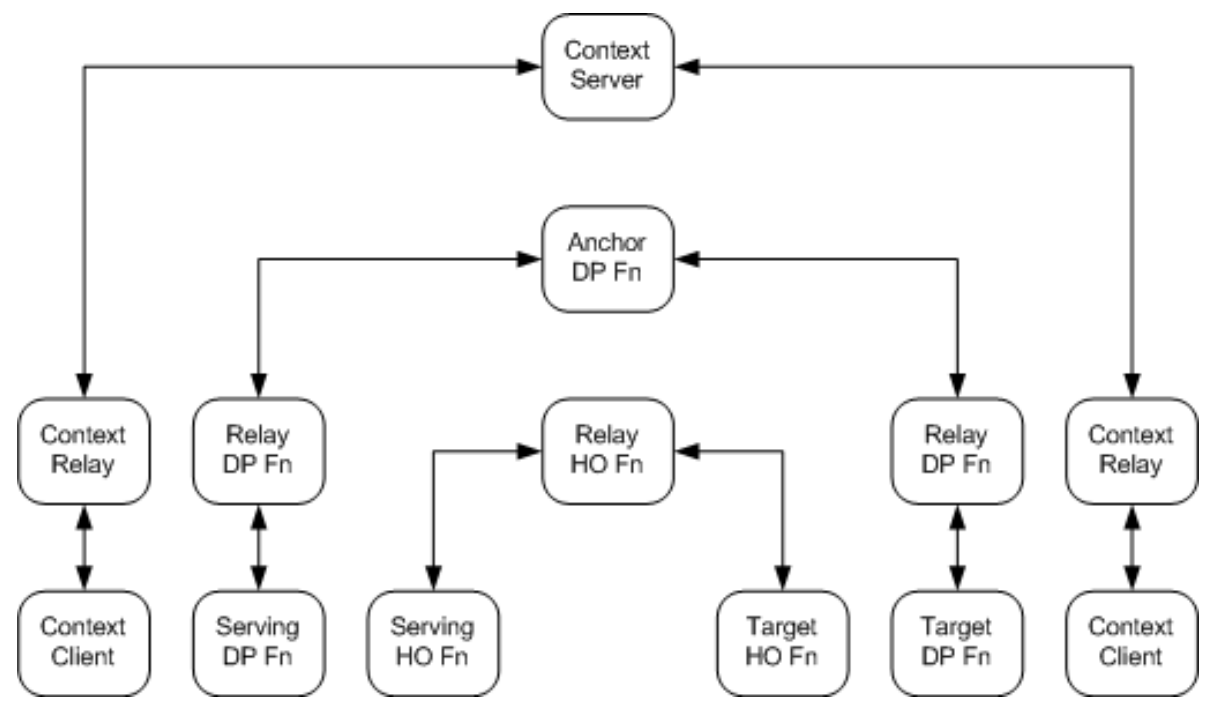

Figure 2.16: ASN anchored mobility functions. 
- Data Path function controls data path establishment and transmission of data packets between entities. A logical division of the Data Path function includes an anchor DP function, a serving DP function, a target DP function and a relaying DP function.

- Handover function controls handover decision, initiation and signaling. Serving $\mathrm{HO}$ function, relaying $\mathrm{HO}$ function and target $\mathrm{HO}$ function constitute the overall Handover function.

- Context function addresses the exchanges required in order to setup any state in network elements during a HO. Context server, context client and relaying context functions control the Context function procedures.

Functions above are hosted by ASNs participating a HO. The functions may be divided into following network elements during a HO process: anchor ASN, serving ASN, target ASN and authenticator ASN (figure 2.17). [14]

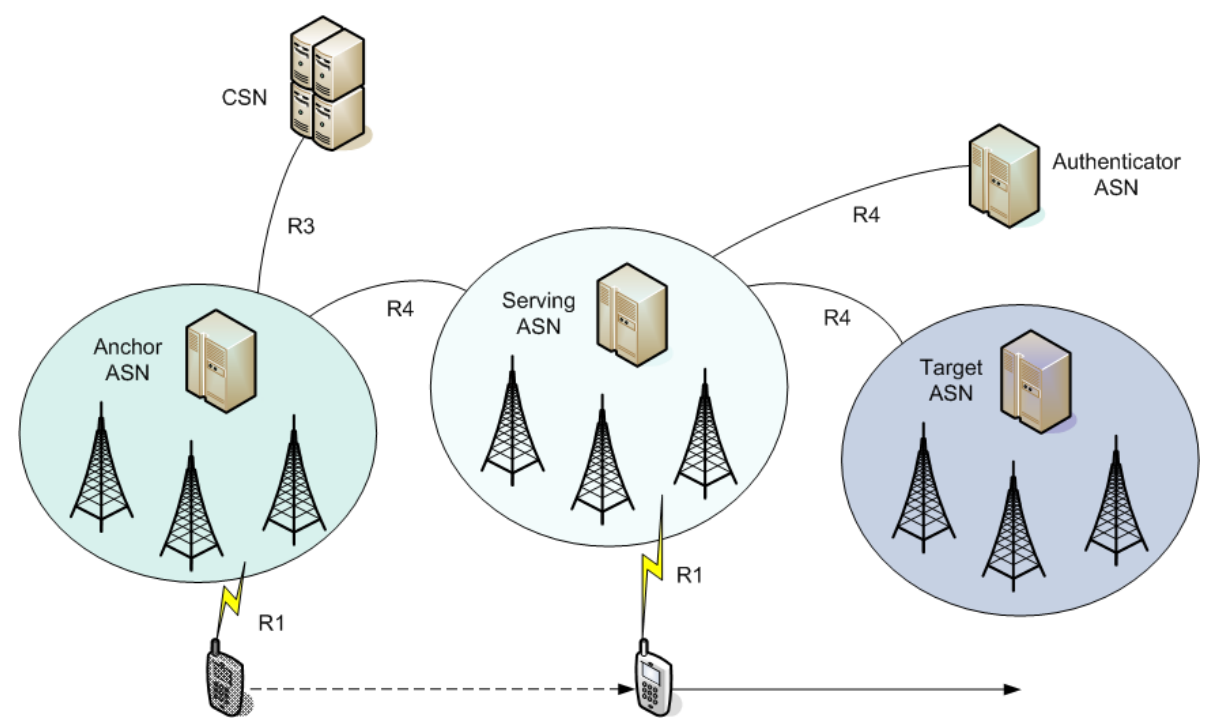

Figure 2.17: ASN anchored mobility scenario.

The anchor ASN is an attachment point to CSN and external network. It hosts the anchor DP function for the MS. Before the handover the MS is connected to the serving ASN which hosts the serving $\mathrm{HO}$ function. The target ASN managing the target $\mathrm{HO}$ function contains a BS that the MS will attach to after a successful handover. After that it will become a new serving ASN for the MS. The Authenticator ASN provides authentication and security functions for the MS. [15][19] 


\subsubsection{CSN-anchored mobility}

CSN-anchored mobility comprises set of procedures associated with MS's movement and change of PoA. In this type of mobility the anchor CSN remains constant but the anchor ASN of a MS may change and signaling and traffic via R3 reference point is exploited. The CSN-anchored mobility means MIP based macro mobility in which re-anchoring of MS from a current FA to a new FA as well as consequent binding updates or MIP re-registration are needed in order to redefinition of data forwarding paths. [14][15]

The R3 mobility may be divided into Intra-NAP R3 mobility or Inter-NAP R3 mobility as in figure 2.18 .

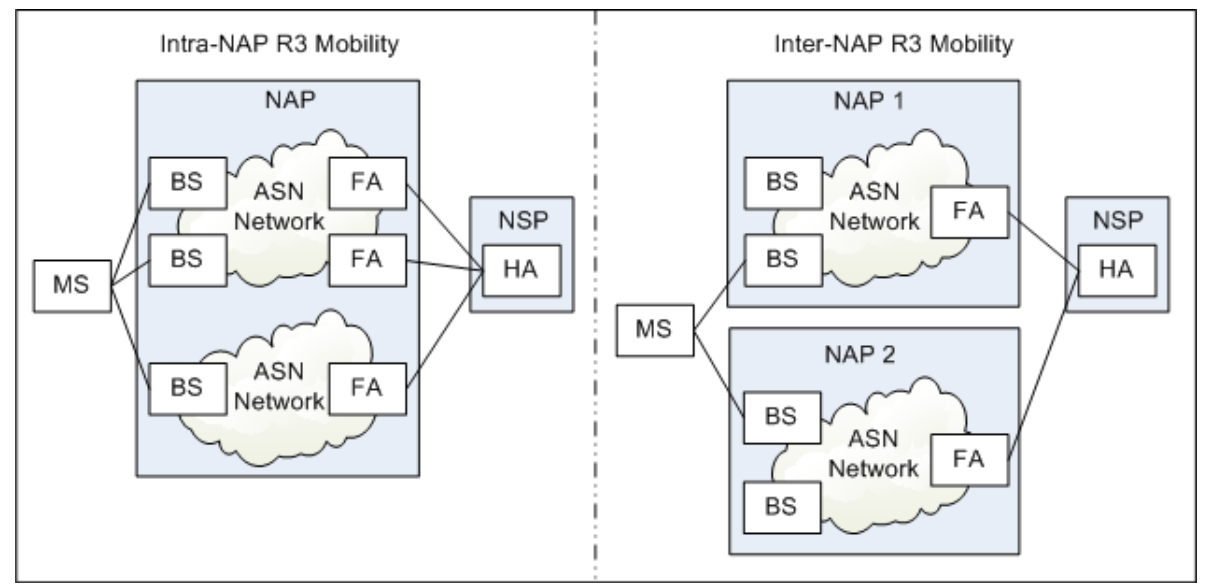

Figure 2.18: CSN anchored mobility.

The inter-NAP R3 mobility enables roaming of the MS between the home NSP and the visited NSP. In non-roaming scenarios the Home Agent (HA) is located in the CSN of the home NSP whereas in a roaming scenario it may reside at the home NSP or the visited NSP. [14]

\subsubsection{Roaming}

Roaming allows users to access wireless services outside their home network by connecting to network maintained by an operator that is not the home network operator. To make roaming possible for the subscribers the home network NSP establishes contracts with other NSPs that allow visitors to operate in their networks and access services that normally would be available only in the home network. Services accessible to a roaming subscriber do not usually differ from the services under the home location, although it depends on the contract made between the NSPs. [23][24] 


\section{Simulations}

\subsection{Introduction}

This section focuses on introducing the reader to the tools used for WiMAX simulations and result examination. Firstly, the Network Simulator 2 (NS-2) is presented which forms a basis for the simulations. Then a WiMAX module called WINSE is introduced which is applied over the basic NS-2 packet to allow WiMAX MAC and PHY simulations. Finally, an ASN-GW extension module for WINSE is divulged. It enables access network functionality and ASN anchored mobility support for the simulations.

Network behavior, performance and features are generally measured through extensive computational testing with different types of simulators which is important for researchers and essential for network and hardware vendors. Traditionally network simulators are categorized into link level, system level and packet level simulators, each of which having different field of function. NS-2 belongs to the last category. [25]

\subsection{Network Simulator 2}

NS-2 is an open source discrete event simulator implemented in C++ and OTcl programming languages. In NS-2 events are controlled by a scheduler and they are performed in chronological order. NS-2 provides a highly modular platform for wired and wireless simulations supporting different network element, protocol, traffic and routing types. [26][27]

The reason for having two programming languages stems from the aim to have an easy to use, yet fast and powerful simulator. Object-oriented C++ forms an efficient class hierarchy core of NS-2 that takes care of handling of packets, headers and algorithms [27]. MIT Object Tcl, or OTcl, is also an object-oriented programming language utilized in NS-2 for network scenario creation, allowing fast modifications to scenario scripts [26]. OTcl and $\mathrm{C}++$ interact with each other through $\mathrm{Tcl} / \mathrm{C}++$ interface called $\mathrm{TclCl}$ as depicted in figure 3.1 [29]. Currently the most recent release of NS-2 is version 2.34 (available at [26]), although a previous version 2.33 is used in this thesis. 


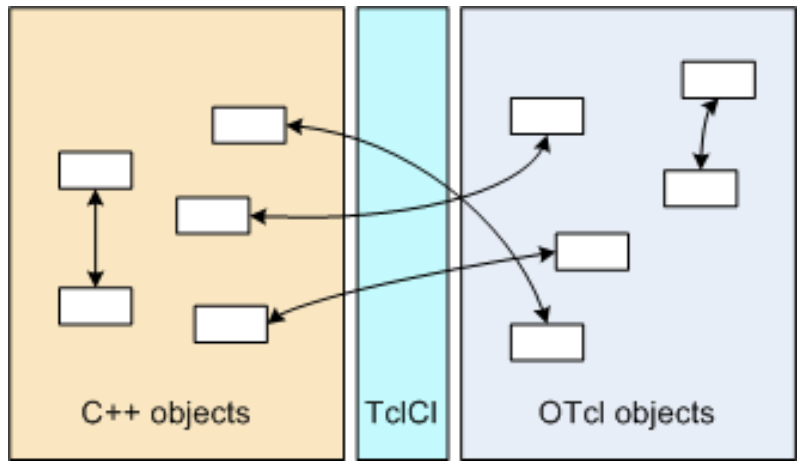

Figure 3.1: $\mathrm{C}++$ and OTcl interaction in NS-2.

In theory using NS-2 is quite straightforward. User creates a simulation scenario with OTcl containing network nodes, applications, topology and connections between the nodes. Each receiving and transmitting node in a scenario contains an agent that is bound to a traffic generator (TCP or UDP application) as a receiving or transmitting end, respectively. The application generates packets that are put to transmission queues in predefined time intervals [27].

NS-2 produces trace files during simulation time which can be post-processed later. A basic trace file may contain event entries, detailed packet information from wired or wireless channel and routing information. Various trace file formats are explained in [28]. Simulation results shown later in this document are mostly gathered from the trace files but occasionally separate runtime logging is needed in order to obtain desired information.

\subsection{WiMAX NS-2 Extension (WINSE)}

WINSE is a WiMAX extension for NS-2 which allows detailed simulation of the air interface between SS, RS and BS as well as intra-node operations. The first WiMAX extensions that lead to the development of WINSE were made on the MAC layer of the Time Division Multiple Access (TDMA) implementation of NS-2 [27]. They focused on QoS enhancements by introducing scheduler and MAC extensions. So far WINSE has been developed by a collaboration project of University of Jyväskylä and Nokia Siemens Networks (NSN). The activities of the project have been focusing on IEEE 802.16 MAC, PHY and access network functionality, all of which are currently being modeled quite in detail. Table 3.1 presents supported features of WINSE. [25]

The most important implemented $\mathrm{C}++$ classes are derived from TclObject class, 
Table 3.1: Features supported by WINSE.

\begin{tabular}{|l|}
\hline PHY \\
\hline OFDM and OFDMa PHY \\
FEC blocks \\
HARQ: Type I, UL ACK channel \\
Channel reports: REP-RSP and CQICH \\
Link adaptation \\
\hline \hline MAC \\
\hline 802.16 d/e/j \\
Duplexing modes: TDD, FDD, H-FDD \\
DL broadcast messages: DL-MAP, UL-MAP, DCD, UCD \\
Compressed MAP, sub-MAPs \\
Connections: DL broadcast, basic management, transport \\
PDU construction, fragmentation, packing \\
Bandwidth requests: standalone and piggy-backed \\
ARQ: blocks, feedbacks, timers, transmission window \\
Uplink contention: OFDM and CDMA-based for OFDMa \\
Network entry \\
Handover \\
Sleep mode \\
\hline \hline QoS and scheduling \\
\hline UGS, ertPS, rtPS, nrtPS, BE \\
BS scheduler \\
SS uplink scheduler \\
Access service network \\
ASN-GW \\
R4, R6, and R8 interfaces \\
ASN-anchored mobility \\
\hline
\end{tabular}

allowing user to easily define desired $\mathrm{C}++$ parameter values via OTcl interface, as well as utilize runtime upcall functions that mitigates OTcl function calls from the $\mathrm{C}++$ core. In addition to well planned class inheritance and internal design the WINSE module provides large amount of command line arguments used for setting parameter values and outputting debugging information. This facilitates running extensive amount of simulations since there is no need for simulation script editing between simulation runs; modifying the command line input parameters is enough. [25] 


\subsection{ASN-GW extension module}

Development of the ASN-GW extension for WINSE was started in 2006 under project title wiper-ho by Nokia Siemens Networks in Beijing [19]. Back then the module contained ASN-GW node implementation. The second significant step of the ASNGW module development was done by Milton Mendieta in co-operation of Nokia and NSN in 2008 [19]. Mendieta released version 0.3 of the module which was compatible with the WINSE release v0.13.2 and the NS-2 version 2.33 [19]. It extended the ASN-GW support to BS node and allowed quite realistic simulations of the access network during handovers.

For this thesis the ASN-GW module was developed further, due to which also the WINSE module was extended to support automatic handover initiation, optimized handover and scanning of the neighboring BSs. Current version of the ASNGW module is v0.3.3 which is applicable to the WINSE v0.15 and the NS-2 v2.33.

\subsubsection{Core files}

The ASN-GW extension consists of one OTcl file, several C++ source files and one patch that extends WINSE files. Next these files and their main purposes are introduced.

\section{ns-wimax-asngw.tcl}

This is the only tcl file in the ASN-GW package. It defines ASN related OTcl classes and instance procedures (instprocs). The most essential classes defined in this file are Agent/ASNAgent, Agent/ASNAgent/BS and WiMAXEncapsulator. The simulator instance procedures create-wimax-asngw $\{$ and attach-to-asngw $\{$ are used for creation of the ASN-GW and binding BS agents to the created ASN-GWs, respectively. The file contains also functions for binding encapsulator and decapsulator objects to ASN agent (ASN-GW or BS). Capsulation operations are needed for packet classification when packets are transmitted from one agent to another.

One important task performed here is storing SSs' anchor ASN-GW information into an internal list. This is done via runtime OTcl upcall from the $\mathrm{C}++$ side by calling set-anchor ASNaddr \{\} instproc. A get-anchorASNaddr-by-id \{\} instance procedure is defined for accessing OTcl container from the C++ to get the identifier of the MS's anchor ASN-GW. 


\section{wimax-asn-defs.h}

This file is dedicated to global definitions and does not perform major operations. During a handover BS and ASN-GW nodes are set to a specific state depending on which handover message they have delivered. These node states and message types are defined in this file. Also durations of the ASN related retransmission times for the handover messages sent over the R4 and the R6 reference points are calculated here. The only actual class declaration in the file is ho_params which keeps, e.g., the handover state of the ASN agent and a copy of the sent packet for possible retransmission. The file contains also definition of the packet buffer by declaring packetbuffer, bufferqueue and bufferlist structures. Data packets are buffered at the anchor ASN-GW when a data path update takes place during a handover.

\section{wimax-asngw. $\{\mathrm{cc}, \mathrm{h}\}$}

These files define ASNAgent, _ASNGW_BINDING_LIST, _ASNGW_BINDING_LIST_ELEMENT and SIGTimer classes. The first one forms a basis for the ASN-GW agent and the BS agent by introducing common methods for both agents. It also defines proprietary functions only for the ASN-GW agent mostly used for message and packet handling. The ASNAgent::attachMobileNode() function saves a binding between a SS and its anchor ASN-GW into the OTcl container described earlier by calling the set-anchorASNaddr\{\} OTcl instproc. Furthermore, the SS-BS engagement is done in this function and stored into __ASNGW_BINDING_LIST linked list. The SIGTimer class is inherited from NS-2's TimerHandler class and it introduces retransmission timer functionality for the implemented access network messages.

It could be generalized that the files introduce handover and data functions for the ASN-GW agent, whereas the next two files do it for the BS agent.

\section{wimax-asnbs.\{cc,h $\}$}

The ASNAgentBS class defined in these files communicates with the MAC layer of the WiMAX BS and it is the only logical link between WINSE and the ASN-GW module if not taken into account mere $\mathrm{C}++$ specific header file inclusions. Similarly to the ASNAgent class the ASNAgentBS implements handover message handling methods, but it also takes care of the inter-BS forwarding over R8. 
wimax-asn-payload.\{cc,h\}

The wimax-asn-payload files contain declarations and definitions for the packet payloads of the handover messages transmitted over the $\mathrm{R} 4$ and the R6. Two payload classes are used: ASNHandoverPayload and ASNPreAttachmentPayload. The first one is utilized with the handover and data path messages, whereas the latter is for the pre-attachment request, response and acknowledgment messages. Both classes use ASNMessagePayload as an upper class, which for its part is inherited from WINSE's WiMAXGeneralPayload class to offer flexible extensibility of payload types.

\section{wimax-encap.\{cc,h\}}

In these files WiMAXEncapsulator and WiMAXDecapsulator classes are composed. They perform IP-in-IP encapsulation and decapsulation which can be commenced by the ASN-GW or the BS agent. Both classes contain a recv() function within which the IP header operations are performed before transmitting a packet to the target. The recv() function performs also packet buffering during handovers if necessary. Instances of the capsulator classes are utilized by the both ASNAgent and ASNAgentBS classes.

\subsubsection{File and class dependencies}

In figure 3.2 can be seen the ASN-GW module file dependencies as well as connections to WINSE files.

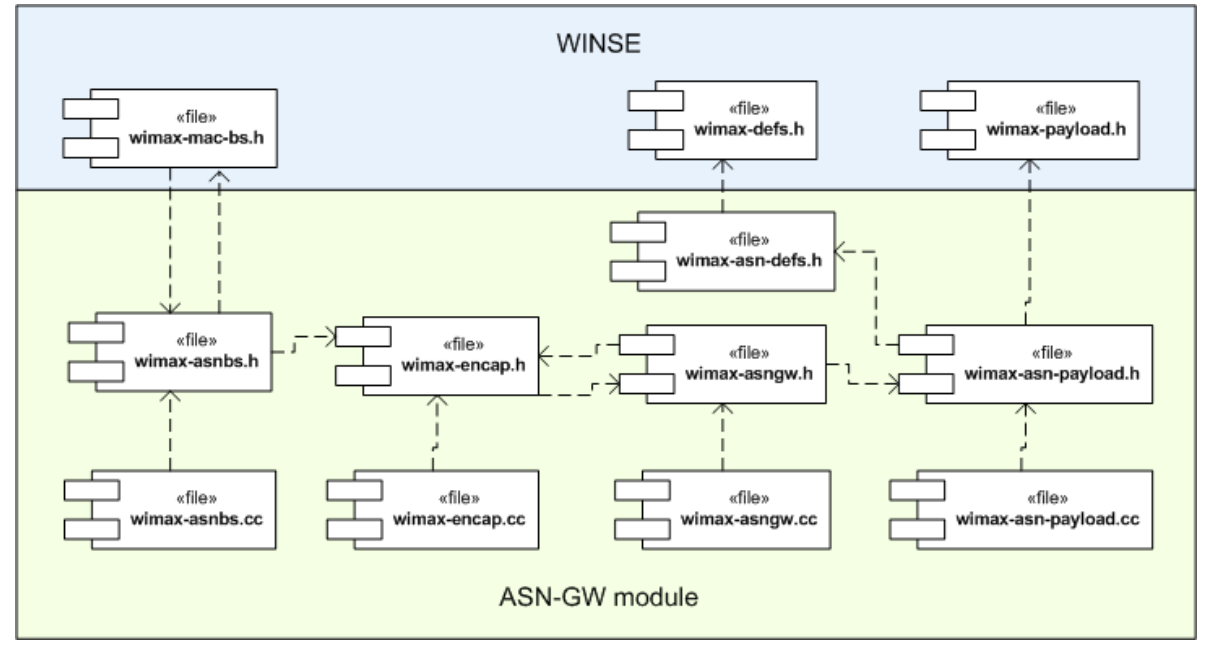

Figure 3.2: File dependencies.

As mentioned before, the link between the BS MAC in WINSE and the BS at ASN 
side is the only explicitly used connection between the modules. The internal design of the ASN-GW module can be improved by removing the circular dependencies between wimax-bs-mac.h and wimax-asnbs.h files and wimax-encap.h and wimax-asngw.h files. This would probably require some reorganization of the class implementations. Currently, include guards used in the beginning of the files allow using the circular dependencies.

Figure 3.3 shows ULM diagram for core classes of the ASN-GW module.

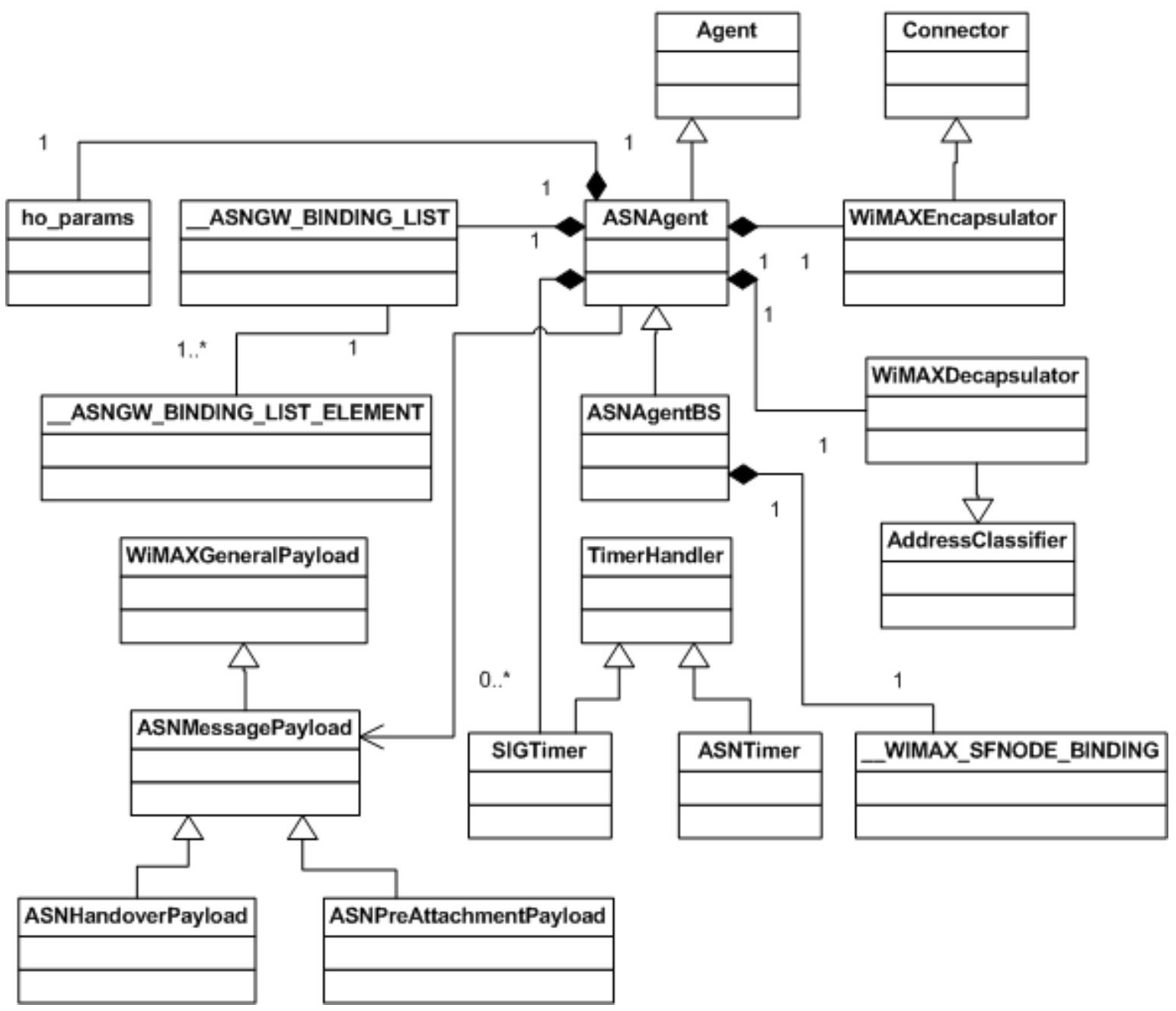

Figure 3.3: UML diagram for ASN-GW module core classes.

As the figure shows, the ASNAgent is the central class around which most of the other main classes are written. As mentioned before, the ASNAgentBS takes care of communication with WINSE, more precisely with WiMAXMAC_BasicBS class (see Appendix B for more information on WINSE classes). 


\subsection{Important simulator features}

In addition to the files, classes and parameters described earlier, there are a few fundamental simulator features that have to be emphasized. This section describes WINSE specific features that are important for the simulations. They either already exist in WINSE or they are applied on WINSE by the patch in the ASN-GW module.

\subsubsection{Scanning of neighbor BSs}

The WiMAX SS node in the simulator is able to scan signal strength levels from the serving BS and the neighboring BSs. This feature is exploited in SSs' network entry phase to determine a BS providing the best signal strength. It is also used in the handover simulations to gather signal level information from all the BSs. The information is then processed for determination of the target BS for a HO. This simplified scanning feature therefore replaces the neighbor advertisement and scanning request messages and the association to the neighbor BSs, which are not implemented in the simulator.

Scanning of BSs' RSSIs is performed at the PHY layer by examining long synchronization preambles before any link adaptation model factors are applied to the received packet. The list of the RSSIs is periodically requested from the handover module of the SS's MAC layer.

\subsubsection{Handover module}

The handover module at the SS MAC consists of functions and variables that allow adjustment of the handover functionality by taking care of hard handover triggering. The handover initiation functionality resides inside one $\mathrm{C}++$ method which provides a good framework for versatile algorithm generation. The module uses sum of the serving BS's RSSI $\left(P_{\text {serv }}\right)$ and the hysteresis margin $P_{\text {hyst }}$, or $P_{\text {serv }}+P_{\text {hyst }}$, as a threshold value for the handover timer triggering. If the signal strength from the neighboring BS $P_{\text {neig }}$ exceeds the $P_{s}+P_{\text {hyst }}$ and is the best among all the BSs, a time-to-trigger timer is started. If the $P_{\text {neig }}$ remains better than the $P_{s}+P_{\text {hyst }}$ during the whole $T_{\text {trig }}$ duration the handover process is initiated with a transmission of the MOB_MSHO-REQ message. If the MS is conducting a handover it will not perform RSSI measurements at that time. 


\subsubsection{Supported management messages}

Successfulness of the network entry and handover procedures rely strongly on the management message exchanges between the SS and the BS. WINSE supports a wide assortment of management messages allowing precise simulations. The network entry and handover message negotiations implemented in the simulator are shown in figures 3.4(a) and 3.4(b).

During an initial network entry the following messages are transmitted over the air interface: UL/DL-MAP for BS scanning, RNG-REQ/RSP, SBC-REQ/RSP, PKM-REQ/RSP, REG-REQ/RSP and DSA-REQ/RSP/ACK for connection creation. When the BS receives a SBC-REQ it starts a MS pre-attachment process with the serving ASN-GW. This three-way handshake is performed via MS_PreAttachment_Req/ Rsp/Ack messages. Pre-attachment is used for defining the anchor ASN-GW and saving the MS-BS binding in the ASN-GW. Service flows created with the DSA exchange are stored at the serving BS agent in the ASNAgentBS class to be re-used in case of handovers.

For the handovers WINSE supports MOB_MSHO-REQ, MOB_BSHO-RSP and MOB_HO-IND management messages. As mentioned before, the neighbor advertisement (MOB_NBR-ADV) messages and the scanning interval request/response (MOB_SCN-REQ/RSP) messages are not implemented. Most of all the handover messages carry ID of the target BS which in the simulator is permanently selected before the MOB_MSHO-REQ is transmitted.

The access network $\mathrm{HO}$ messages transmitted between the BSs and the ASNGWs were explained in the section 2.10.2, although the simulator implementations of the messages are a bit stripped for the sake of simplicity. Actually their main task is to tell the network entities taking part of the handover which phase of the $\mathrm{HO}$ is ongoing. Moreover, the HO_Req sent by the serving BS is used for carrying service flow information to the target $\mathrm{BS}$ for it to be able to create connections after the $\mathrm{HO}$ without the DSA exchange.

A network entry after a handover omits exchanges of the SBC and PKM messages. This speeds up the network re-entry. After the ranging handshake registration messages are transmitted. When the BS receives a REG-REQ it revives the MS's service flows, creates transport connections for them and finally includes the service flow list in a REQ-RSP. The MS obtains the service flow information from the response message and also sets up new connections for its part. 


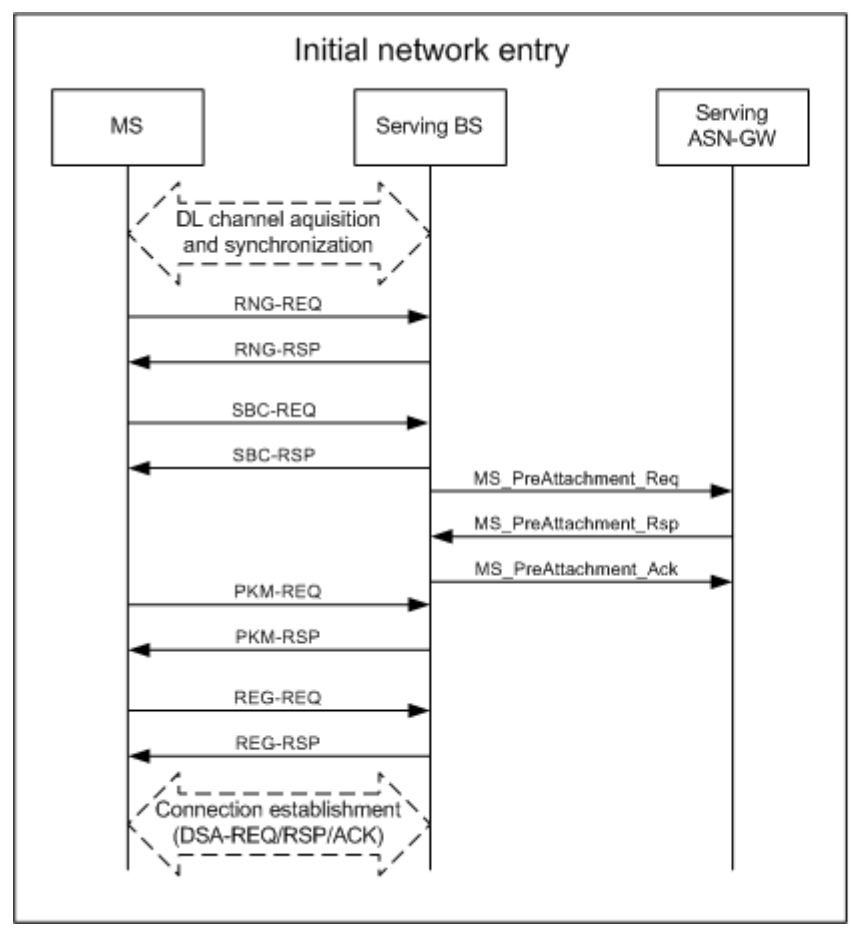

Post-handover network re-entry

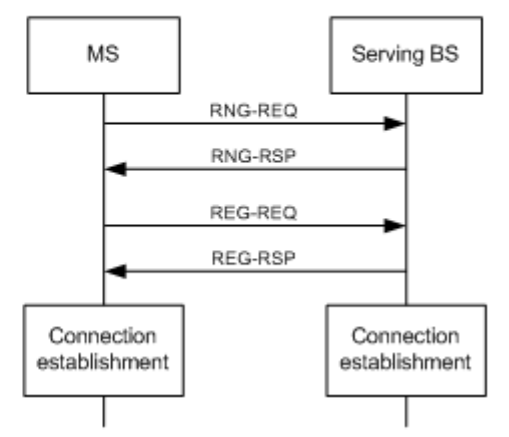

(a) Network entry messages.

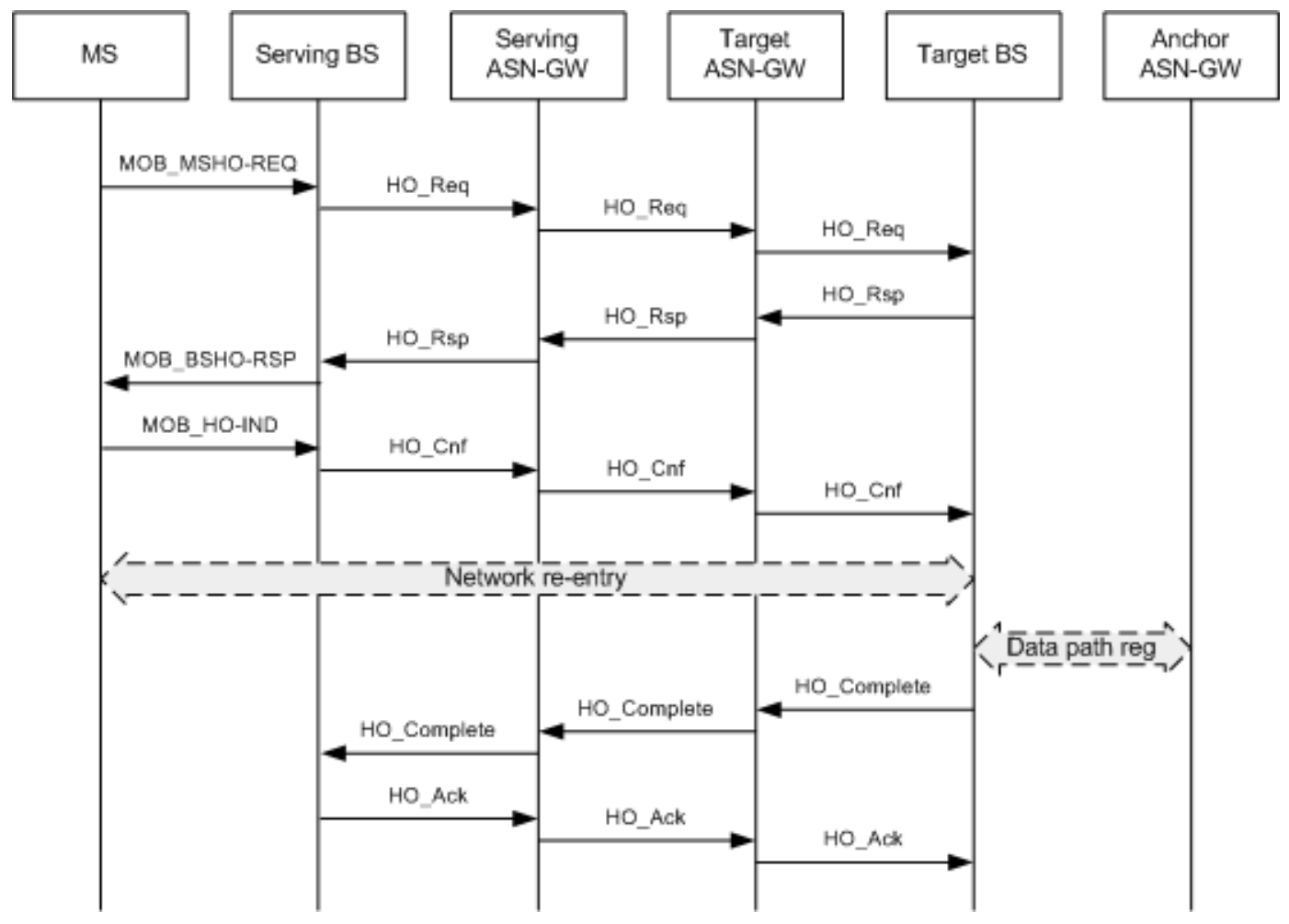

(b) Handover message exchanges.

Figure 3.4: Network entry and handover implementations. 


\subsubsection{Handover timers}

Three handover timers are implemented. First one, the time-to-trigger timer, was introduced in section 3.5.2.

The second timer is called MS_handover_retransmission_timer $\left(T_{m s h o}\right)$ used for scheduling MOB_MSHO-REQ message retransmissions. It is started at the transmission phase of the handover request message. If the MS running this timer does not receive a MOB_BSHO-RSP from the serving BS during the duration of the $T_{m s h o}$ a new request message is sent. There is only a limited number of the retransmissions since it is important to reconnect as fast as possible to the new BS in order to reduce packet drops and communication outages. So, if the maximum amount of retransmissions is consumed, it can be presumed that the MS is in such bad condition that it will not able to complete the HO successfully. Therefore, in this case the MS removes connections with the serving BS and starts scanning of new BSs. After that it performs a new initial network entry to the most powerful BS. In the simulator this is actually the only incident that causes a failed HO.

The third timer, HO-IND timer $\left(T_{\text {hoind }}\right)$, resides at the BS. When the MOB_BSHORSP is transmitted a new $T_{\text {hoind }}$ is created and started. There might exist multiple simultaneous handovers at the same BS, thus a single timer would not suffice here. Transmission of the MOB_BSHO-RSP means that the pending $\mathrm{HO}$ will be carried out all the way for BS's part despite it receives a MOB_HO-IND or not. This way a $\mathrm{HO}$ can be successful in case the MS receives the MOB_BSHO-RSP but the MOB_HO-IND is dropped. According to the specification, this would be the case also in reality, although the specification defines also additional messaging at the access network [8].

\subsection{Simulation scenario}

The target for the simulations is to generate handovers by moving the mobile nodes within a specific area where they might cross the borders of the sectors. Trajectories of the MSs are not planned, but they are rather sporadic in each simulation run. Quite excessive amount of simulations are needed for obtaining reliable results and minimizing the effect of outliers. For this purpose seeding of simulations is used. Changing the seed value of NS-2 creates alteration in otherwise similar simulations.

Figure 3.5 shows the network topology used in the simulations. It contains three BSs using directional antennas and each of them produce sectors that cover 120 degrees. The inter-station distance (ISD) is 1000 meters. 


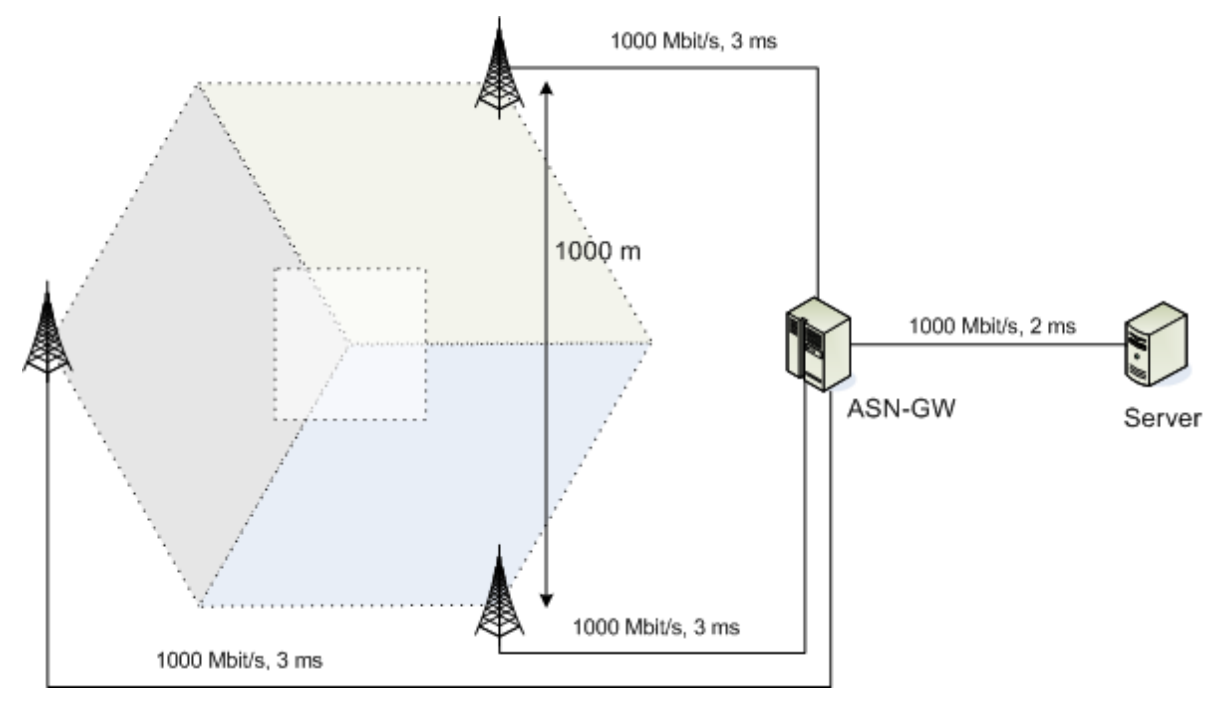

Figure 3.5: Network topology for the simulations.

All three BSs are connected to the same ASN-GW with $1 \mathrm{Gbit} / \mathrm{s}$ wired links with 3 ms latency. The ASN-GW is attached to the server with a 1 Gbit/s, 2 ms wired connection. Mobile stations move inside the rectangular area in the middle of the BSs' coverage areas. The size of the movement area is quite limited in order to keep the simulation time small and to increase the number of HOs. Keeping the simulation time small emphasizes the impact of HOs to overall network performance. Movement of the MSs is randomized as well as their starting locations. Therefore some of the MSs might not perform handovers at all during one simulation run. [36] defines MS trajectory proposals for simulation purposes. They cover MS's movement across two cells, movement along the cell border and a trajectory where the MS first moves along the border of two cells and then moves under the coverage of third cell. With the topology depicted in figure 3.5 all these trajectories can be modeled, although such specific simulations are not created. Complete OTcl simulation script used in this thesis can be found in Appendix C.

In the simulations UDP traffic is generated in both DL and UL direction. Altogether $20 \mathrm{MSs}$ is used in the scenario. All of them have constant bit rate (CBR) UDP applications creating Best Effort (BE) traffic at the rate of $100 \mathrm{kbit} / \mathrm{s}$. By setting this data rate for users located near the cell edge it is possible that the BSs may not be able to serve all the MSs with a full success rate. This is due to increased number of packet retransmissions and enforced use of robust MCSs for the bursts which produce much overhead.

In the simulations impact of variable length time-to-trigger timer to the network performance is inspected. Four time-to-trigger values are used, namely $0.05 \mathrm{~s}, 0.1 \mathrm{~s}$, 
$0.25 \mathrm{~s}$ and $0.5 \mathrm{~s}$. As the channel measurements are performed in every frame in the simulator, it is also possible to start the time-to-trigger timer in every frame. Frame duration is $5 \mathrm{~ms}$, so if the time-to-trigger is set to a very small value it is presumable that handovers back and forth between BSs will occur. Four velocities for the MSs are used: $2 \mathrm{~m} / \mathrm{s}, 5 \mathrm{~m} / \mathrm{s}, 10 \mathrm{~m} / \mathrm{s}$ and $20 \mathrm{~m} / \mathrm{s}$. Purpose of different speeds is to show the impact of the velocity to handover functionality. As will be seen later the velocity affects explicitly, for example, to the number of handovers. For each combination of time-to-trigger and MS velocity twelve different seeds are used. In total num $_{\text {time-to-trigger }} \cdot$ num $_{\text {velocity }} \cdot$ num $_{\text {seed }}=4 \cdot 4 \cdot 12=192$ simulations were ran.

In addition to changeable time-to-trigger and MS velocity, various other factors affect the behavior of the network and HO either explicitly or implicitly. Most important MAC, PHY and hardware related constant simulator parameters and values are shown in table 3.2. Some parameters worth noticing relate to HARQ and the air link modeling. Type I HARQ is used in which blocks received multiple times are combined and fed to the FEC decoder. Each HARQ retransmission improves a chance of successful assembling of undecayed data. Maximum number of HARQ retransmissions is four and if it is exceeded the packet is completely lost. Many parameters affect the wireless channel properties. $10 \mathrm{watt}$ transmission power is used at the BSs and 0.25 watts at the MSs. An urban macro-cell (UMa) environment is utilized with a trace file based fast fading modeling. A constant $3 \mathrm{~dB}$ hysteresis margin is required for the handover initiation.

Table 3.3 lists retransmission times for the management messages transmitted during the network entry. These are very crucial for understanding the handover performance. If a certain management message is lost, the timeout value will trigger a message retransmission process. As mentioned before, the MOB_MSHO-REQ is the only message that can be transmitted multiple times during a HO preparation. Retransmission timeout for which is set to $30 \mathrm{~ms}$. WiMAX specifications usually define minimum and maximum values for the message retransmission times. In the simulator timeouts from the shorter side are selected in order to speed up the network entries. Long retransmission times would decrease the performance significantly since bad channel conditions and packet drops are anticipated.

Simulations with different velocities are conducted in a way where the simulation time depends on the velocity. A basic simulation time of 300 seconds is used which is divided by the velocity. As a result, during each simulation run a MS moves the same distance regardless of its velocity. This type of approach makes simpler a comparison of handover functionality with varying velocity when compared to a constant simulation time for all the velocities. 
Table 3.2: 802.16 network parameters.

\begin{tabular}{|c|c|}
\hline Parameter & Value \\
\hline Center frequency & $2.5 \mathrm{GHz}$ \\
\hline Reuse factor & $1 / 3$ \\
\hline Bandwidth & $10 \mathrm{MHz}$ \\
\hline PHY & OFDMa \\
\hline Duplexing mode & TDD \\
\hline Frame duration & $5 \mathrm{~ms}$ \\
\hline Channel report type / interval & $\mathrm{CQICH} / 20 \mathrm{~ms}$ \\
\hline Channel measurements DL/UL & preamble / data burst \\
\hline Channel measurements filter & EWMA, $\alpha=0.25$ \\
\hline Link adaptation model & target FEC BLER, $10^{-1}$ \\
\hline Antenna technique & SISO \\
\hline BS / SS Tx power & $10 / 0.25 \mathrm{~W}$ \\
\hline BS / SS antenna pattern & 3GPP / omni \\
\hline BS / SS antenna height & $32 / 1.5 \mathrm{~m}$ \\
\hline Inter-station distance & $1000 \mathrm{~m}$ \\
\hline path loss & $.16 \mathrm{~m} \mathrm{UMa}$ \\
\hline fast fading $\mathrm{K}$ factor & $0 \mathrm{~dB}$ \\
\hline handover hysteresis margin & $3 \mathrm{~dB}$ \\
\hline time-to-trigger & $0.05,0.1,0.25,0.5 \mathrm{~s}$ \\
\hline HARQ & Type I (CC) \\
\hline HARQ channels & 16 \\
\hline HARQ buffer size & 2048 B (per channel) \\
\hline HARQ shared buffer & $\mathrm{ON}$ \\
\hline HARQ max. retransmissions & 4 \\
\hline HARQ ACK delay & 1 frame \\
\hline PDU SN & $\mathrm{ON}$ \\
\hline PDU SN type & long ( 2 bytes) \\
\hline Ranging transm. opport. & 2 \\
\hline Ranging backoff start/end & $1 / 15$ \\
\hline Request transm. opport. & 2 \\
\hline Request backoff start/end & $0 / 15$ \\
\hline PDU size & Unlimited \\
\hline Packing/Fragmentation & $\mathrm{ON}$ \\
\hline
\end{tabular}

Table 3.3: Retransmission times for management messages.

\begin{tabular}{|l|c|}
\hline Management message & Retransmission timeout [ms] \\
\hline RNG-REQ & 10 \\
SBC-REQ & 50 \\
PKM-REQ & 50 \\
REG-REQ & 10 \\
DSA-REQ & 200 \\
DSA-RSP & 100 \\
MOB_MSHO-REQ & 30 \\
\hline
\end{tabular}

\subsection{Simulation results}

With the simulation scenario used it is possible to study multiple network and handover statistics. Observations during the simulations are done for a single han- 
dover and also for the overall network performance. First, gathered network entry and handover times are shown and explained after which other obtained network statistics are depicted.

For the sake of clarity the following terms are used in this section for different types of network entries:

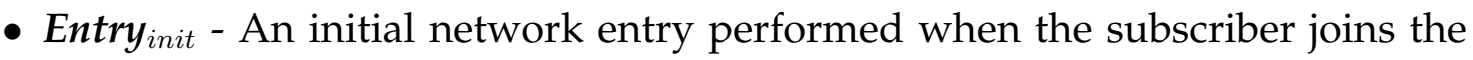
network for the first time. This may occur, for example, when the mobile equipment is turned on. Before the entry process is started scanning of nearby $\mathrm{BSs}$ is required at the MS to determine the BS it should attach to.

- Entry $_{h o}$ - A network entry to the target BS after a successful handover. This type of re-entry is usually much faster than the Entry init $_{\text {since less signaling }}$ between the MS and the BS is needed to accomplish the operation, as shown in the figure $3.4(\mathrm{a})$.

- Entry $_{\text {fail }}$ - A network entry performed after a failed handover. In practice this

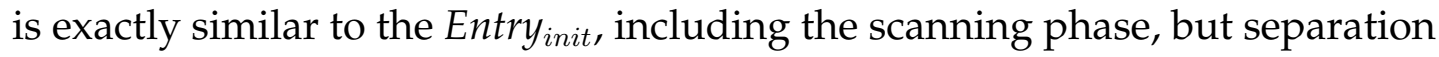
of these two types is required for the results explanations.

Figure 3.6 contains results for the Entry init $_{\text {and Entry }}$ ail durations. Results for both types are combined here since the procedures are practically similar. Time periods shown with the cumulative distribution function (CDF) graph are measured from the transmission of the RNG-REQ message from MS to BS to the transmission of the REG-RSP from BS to MS (see figure 3.4(a)).

Changing the time-to-trigger timer value (indicated with the TTT values in the figure legend) does not have any impact on Entry init $_{\text {times but might affect the Entry fail }}$ times. The figure shows that each network entry lasts at most 1 second and over 90 $\%$ of network entries stay under 0.4 seconds with each velocity. Small differences that occur with different TTT values with the same velocity result from network entries performed after failed handovers. As the TTT affects the moment of the HO initiation, it therefore affects the Entry fail initiation time. With a longer TTT the MS needs to be connected to the old BS for longer time than with a smaller value. This results in MS communicating over a worse channel for increased time before it can migrate to the new BS and better channel. Now, after a failed $\mathrm{HO}$, the longer the TTT the closer the MS is to the new BS and a better channel will be available. Thus less management message retransmissions is needed and the network entry will not be delayed. Steps starting approximately at 0.05 seconds come from varying amount of management message retransmissions (see retransmission timeouts in table 3.3). 


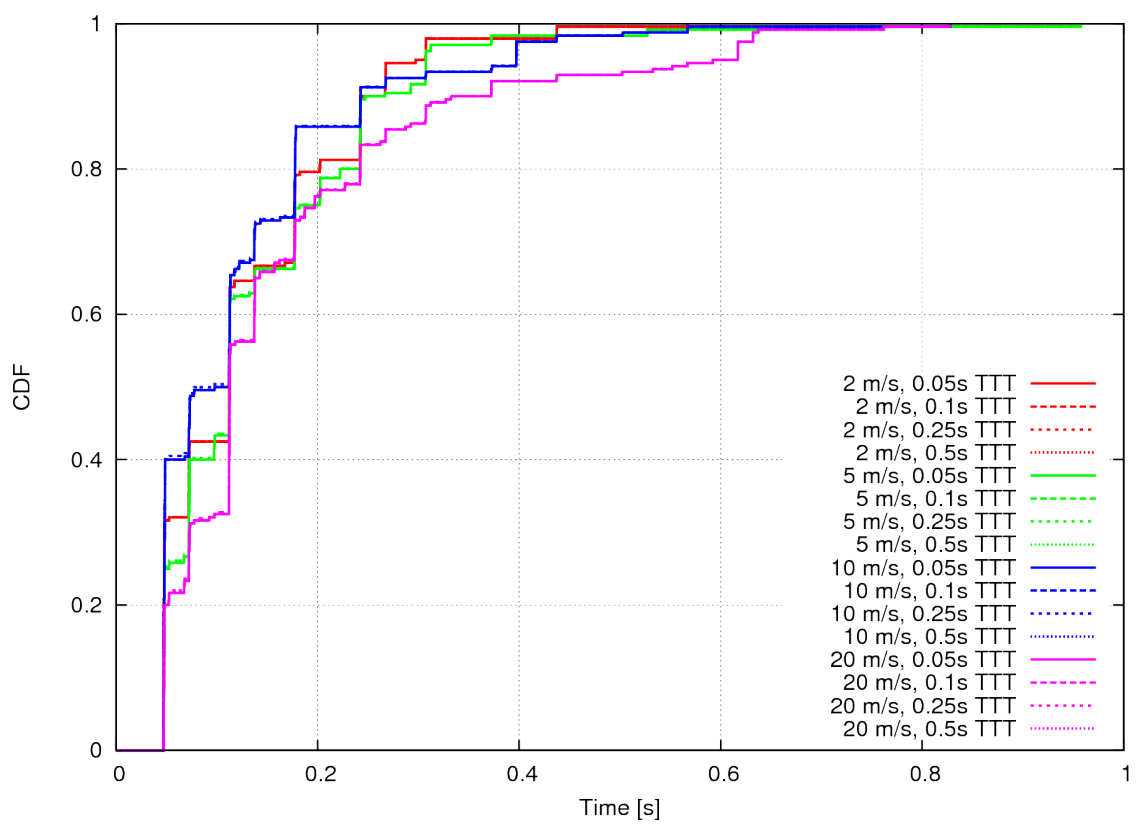

Figure 3.6: Network entry durations.

Durations shown in the figure are quite reasonable for the initial network entries. For example, when the mobile device is turned on, a network entry lasting for half a second basically does not increase the startup time of the equipment. However, in case of Entry fail these durations are not desirable since they cause quite long interruptions which are perceptible with real-time applications. Avoidance of handover failures is therefore a very advisable goal when designing $\mathrm{HO}$ functionality.

Figure 3.7 shows the number of successful and failed handovers performed during the simulations. In total, there were 2088 handovers and five of them failed, so the handover failure rate is approximately $0.24 \%$. As expected, the total number of HOs decreases with a longer $T_{\text {trig }}$ value and there might be simulation runs that do not have any handovers. An explanation for a larger number of HOs with $2 \mathrm{~m} / \mathrm{s}$ velocity and $0.05 \mathrm{~s} T_{\text {trig }}$ arises from the fast fading behavior. Figure 3.8 shows RSSI levels from two BSs measured by a MS moving at the speed of $2 \mathrm{~m} / \mathrm{s}$. It can be observed that occasionally the fast fading does not vary fast enough thus resulting in a situation where the channel measurement and the link adaptation can follow channel variations. It is obvious that such a small $T_{\text {trig }}$ would not be suitable for small subscriber velocities which would usually be the case, for instance, in city centrums or shopping malls. With higher velocities fast fading alters more rapidly where the channel measurement process just follows an average. Therefore, HOs do not occur so often even with a small $T_{\text {trig }}$. 


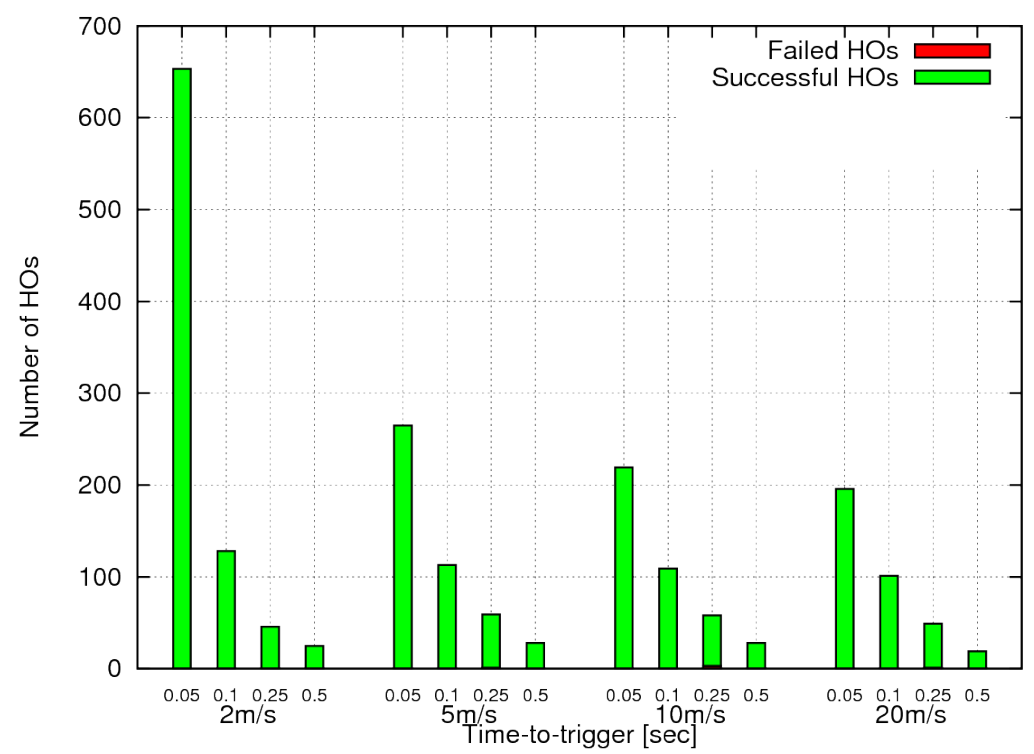

Figure 3.7: Total number of handovers.

A BS switching is preceded by a handover preparation phase starting from transmission of the MOB_MSHO-REQ message. The preparation is concluded with a MOB_HO-IND transmission, after which the MS deletes old connections. New connections are established after the network re-entry and registration to the target BS. Therefore the time gap between the MOB_HO-IND and the REG-RSP messages (handover interruption time) has a direct impact on packet delays and throughput. Durations of HO preparation ${ }^{1}$ and interruption ${ }^{2}$ times are depicted in figure 3.9 and figure 3.10, respectively. The figures contain results only for the successful handovers since the transmission of the MOB_HO-IND is not performed in case of failed HO.

It can be seen from figure 3.9 that there is no relevant correlation between a particular $T_{\text {trig }}$ value and handover preparation time. It is rather the MS's velocity that impacts the rapidness of the handover preparation process. Indeed, the time needed for the handover handshake process depends merely on a fact that the handover messages are transmitted successfully, which, in turn, depends on the MS speed and other mechanisms, such as channel measurements, link adaptation, scheduling etc. Although the preparation times are quite small and do not interrupt a data transmission process, there seems to be lots of message retransmissions triggered ei-

\footnotetext{
${ }^{1}$ Handover preparation time is measured from transmission of the MOB_MSHO-REQ to transmission of the MOB_HO-IND message.

${ }^{2}$ Handover interruption duration is measured from transmission of the MOB_HO-IND to transmission of the REG-RSP.
} 


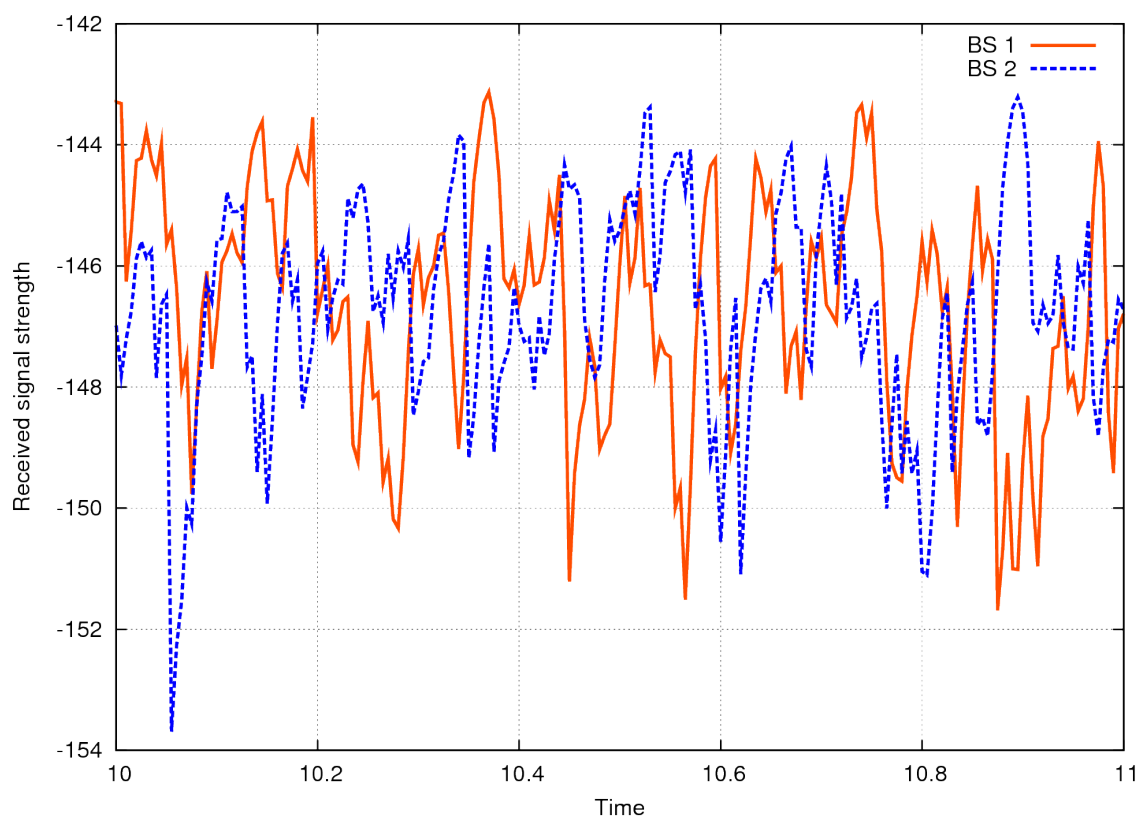

Figure 3.8: Impact of fast fading on RSSI levels.

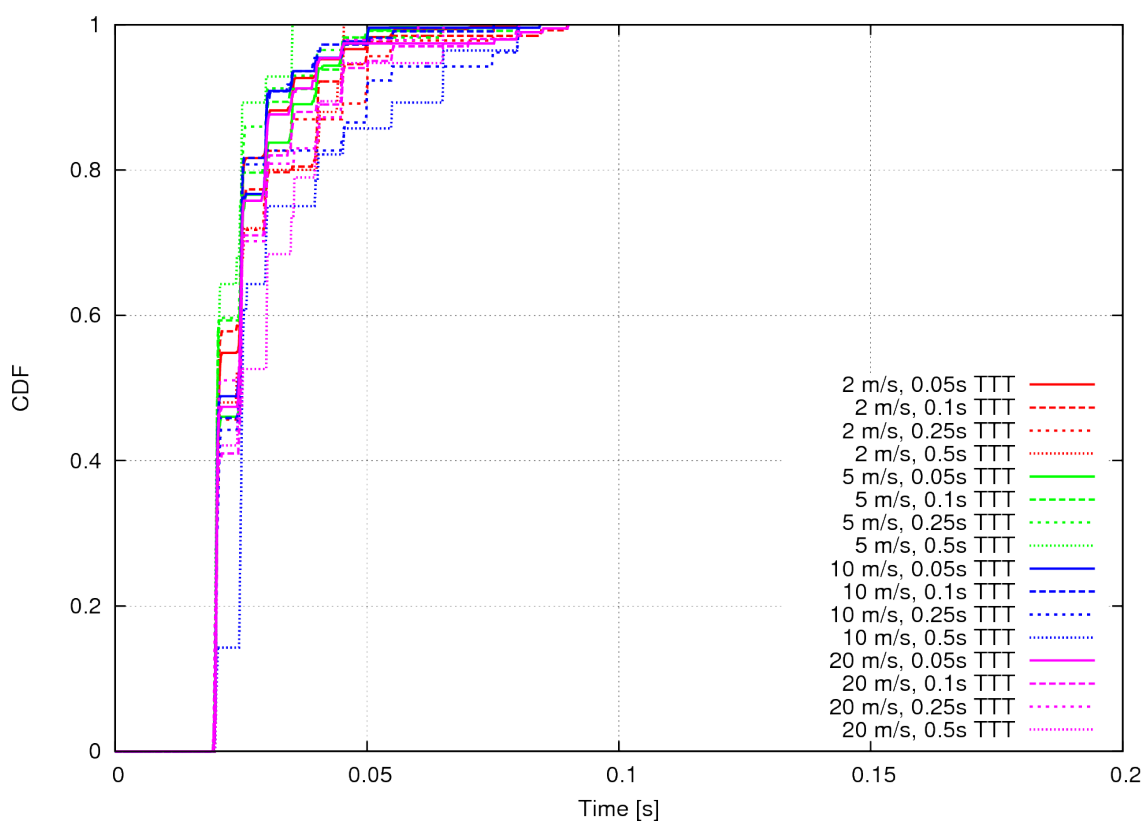

Figure 3.9: Handover preparation time.

ther by the retransmission timers or the HARQ. This will increase overhead that, at the cell edge, will consume a large portion of the available bandwidth even without handovers. At least UDP traffic with even moderate data rate demand will suffer at this stage from excessive signaling. 


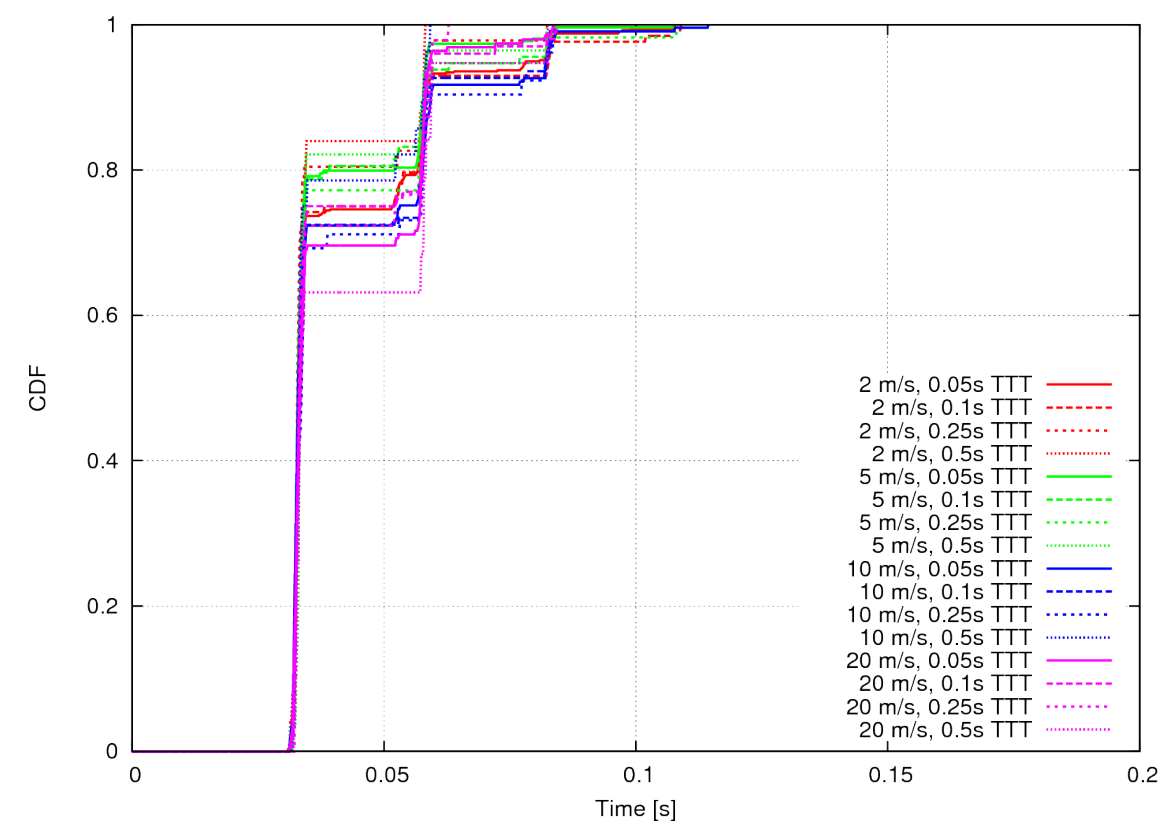

Figure 3.10: Handover interruption durations.

Similarly to the handover preparation, the handover interruption times in figure 3.10 do not correlate with different $T_{\text {trig }}$ values. The interruption times do not exceed 0.12 seconds and at least $60 \%$ of the post-handover network entries can be performed approximately within 30 milliseconds. With VoIP, such a disruption time should not decrease the quality of the connection excessively. For an explanation of the durations in the figure mere knowledge of retransmission timeout values is not enough, a detailed inspection of the network entry signaling is needed also. Figure 3.11 shows two examples of network entry related message exchanges after a handover.

The procedure in the upper figure shows an optimal interruption time, that is, the entry process without message drops. From this figure it is clear that the interruption time is always at least $30 \mathrm{~ms}$. In the lower figure the RNG-REQ message is retransmitted once, increasing the network entry and interruption times by approximately $20 \mathrm{~ms}$. This is applicable also to subsequent retransmissions.

Total handover time, which is the sum of durations shown in figures 3.9 and 3.10 , could be reduced by utilizing bandwidth pre-allocation mechanisms for $\mathrm{HO}$ messages. In addition, interruption times could be decreased with fast ranging, which was not applied in the simulations. With fast ranging, it is possible to hasten the re-entry process at least by $5 \mathrm{~ms}$ by pre-allocating bandwidth for the ranging request. Nevertheless, a clear advantage of the optimized handover to network en- 

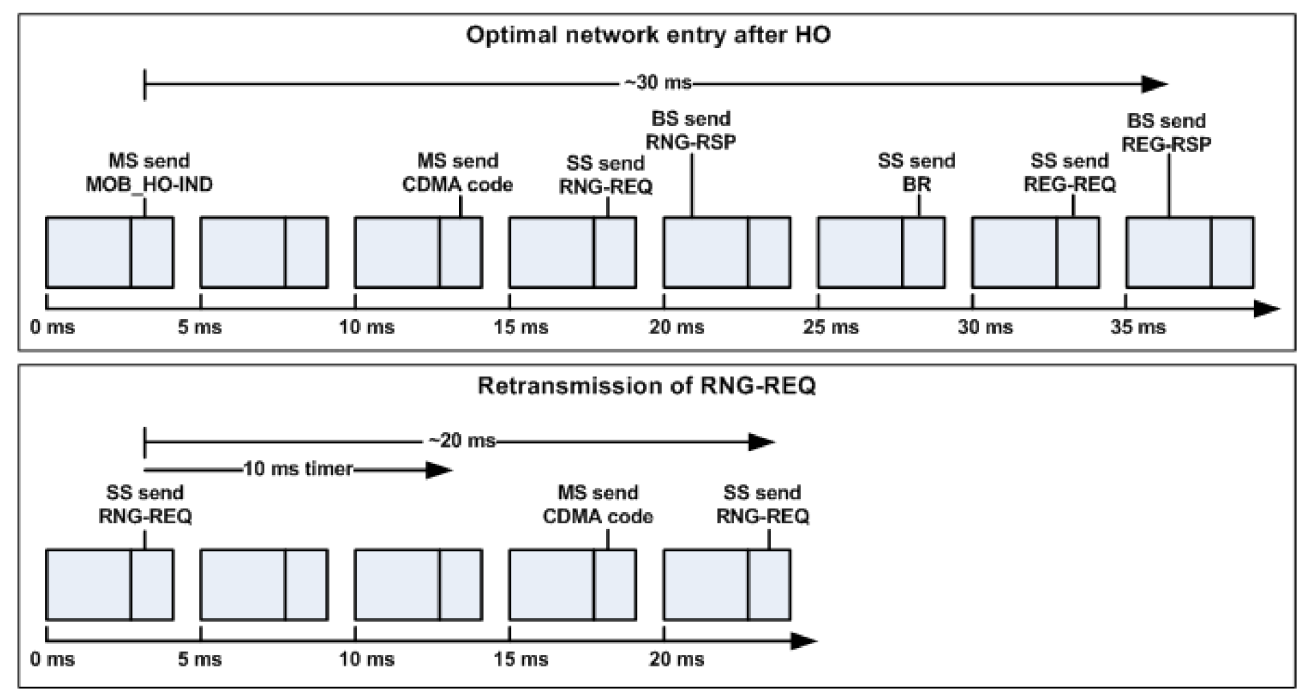

Figure 3.11: Network entry signaling during handover.

try durations can be seen when comparing initial network entry durations in figure 3.6 to re-entry times after handovers in figure 3.10. Durations in both figures are quite comparable since the measurements for figure 3.6 begin from the transmission of RNG-REQ and for figure 3.10 from the transmission of MOB_HO-IND, which is immediately followed by a transmission of the RNG-REQ message.

So far the findings have been focusing on the handover process itself. The impact of different $T_{\text {trig }}$ values to overall network activity may also vary considerably. In figure 3.12 the distribution of the Modulation and Coding Schemes (MCS) used for transmitted bursts can be seen when stations move at $20 \mathrm{~m} / \mathrm{s}$ velocity. For lower speeds similar behavior occurs, thus they are not shown here. In the simulator, 64QAM, 16QAM and QPSK modulations and coding rates of 5/6, 3/4, 2/3 and 1/2 are utilized. Furthermore, QPSK1/2 may use the repetition factors of 2,4 or 6 . The most efficient MCS, 64QAM5/6, is located to the leftmost side of the figure and the robustness of the MCSs grow towards the right side, QPSK1/2 with the repetition factor of 6 being the most robust.

The $T_{\text {trig }}$ value of $0.05 \mathrm{~s}$ provides highest proportion of bursts that are able to use 16QAM MCSs which seem to be the most efficient MCSs available for the cell edge users in the simulation scenario. Better results for the smaller $T_{\text {trig }}$ values can be explained partly when taking into account the time the handover is performed. With $0.5 \mathrm{~s} T_{\text {trig }}$ a MS may need to be connected to the old BS even several seconds longer than with $0.05 \mathrm{~s} T_{\text {trig }}$ before being able to make a handover to the new BS that provides a better channel. In the simulations there were differences of more than seven seconds in this. It is obvious that during this time a MS using a small 


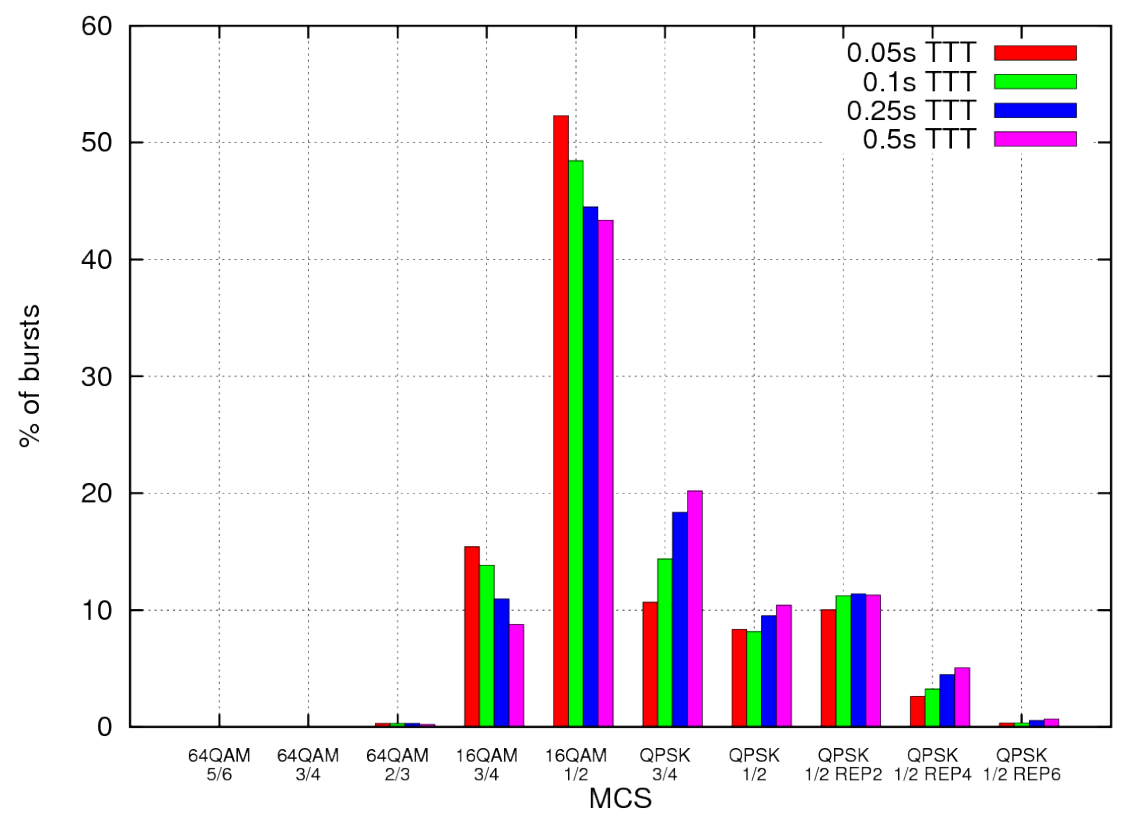

Figure 3.12: Distribution of MCSs at $20 \mathrm{~m} / \mathrm{s}$ MS velocity.

$T_{\text {trig }}$ and migrating to better channel fast is able to utilize a higher amount of more efficient MCSs for its transmissions. Meanwhile a user with a longer $T_{\text {trig }}$ has to communicate through the old BS over a worse channel, thus being forced to use more robust MCSs.

The HO initiation time is not the only reason for such MCS distribution. As mentioned before, with a short $T_{\text {trig }}$ the RSSI measurement is able to follow the channel fluctuation caused by fast fading. This applies especially in small velocities. In such a situation the MS can migrate rapidly between BSs multiple times to acquire a better channel. Nevertheless, one needs to remember the amount of connection drops with a small $T_{\text {trig }}$ due to numerous handovers.

\section{Need for additional HO triggering mechanisms}

Although in this thesis only differences in RSSI levels might trigger the handover, it is important to remember other possible reasons for $\mathrm{HO}$ triggering. Imagine a situation where most of the users are attached to the same BS. There could be neighbor BSs able to serve some proportion of the users, but due to best signal from the most nearby BS the users have selected it to be their attachment point. The user distribution between BSs in this case is likely to be somewhat unfair when considering the traffic load the BSs need to handle. This type of scenario is discussed in the following paragraphs. In this section no handover triggering algorithms are provided 
or implemented, but the information provided here may rather be used for further development of handover triggering criteria.

Outcome of a single simulation run is exhibited here to present the need for load balancing between BSs. This example simulation scenario is similar to those used for gathering the results explained earlier. In the scenario 20 users move inside the three sectors at the speed of $20 \mathrm{~m} / \mathrm{s}$. Simulation time is 10 seconds, $T_{\text {trig }}$ duration is 0.05 seconds and other parameters are similar to those listed in tables 3.2 and 3.3. In figure 3.13 can be seen the number of subscribers served by each of the BSs.

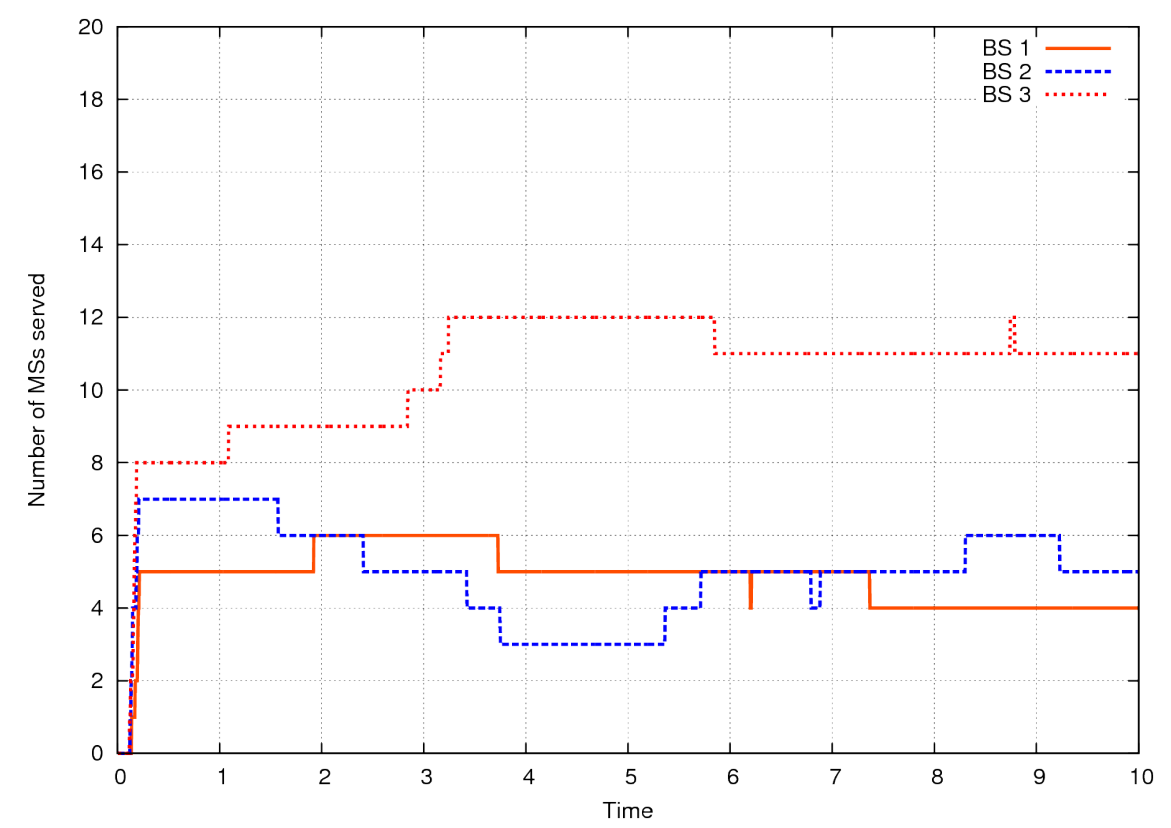

Figure 3.13: Number of MSs served by BSs.

Approximately half of the MSs are connected to the BS 3 and the other half of the users are distributed quite evenly between the BS 1 and BS 2. Numbers shown here are gathered from BSs' internal SS lists. Total number of MSs is 20, but handovers may cause that a single MS might be included simultaneously in more than one internal SS list at different BSs. This results in temporal uneven amount of MSs, which can be seen, for example, at $2 \mathrm{~s}$, where the total number of served MSs seems to be 21 instead of 20 .

One might think that such amounts of served MSs should not cause any difficulties for the BSs. Now, let us remember that for each of the MSs UDP data rates of $100 \mathrm{kbit} / \mathrm{s}$ were used in DL and UL. As the BS uses common transmission buffer for all the MSs it serves, such data rate might rapidly increase the congestion levels in the downlink packet queue. The total sizes of DL queues during the example sim- 
ulation run are depicted in figure 3.14. The applications used in this scenario start transmissions at $1 \mathrm{~s}$.

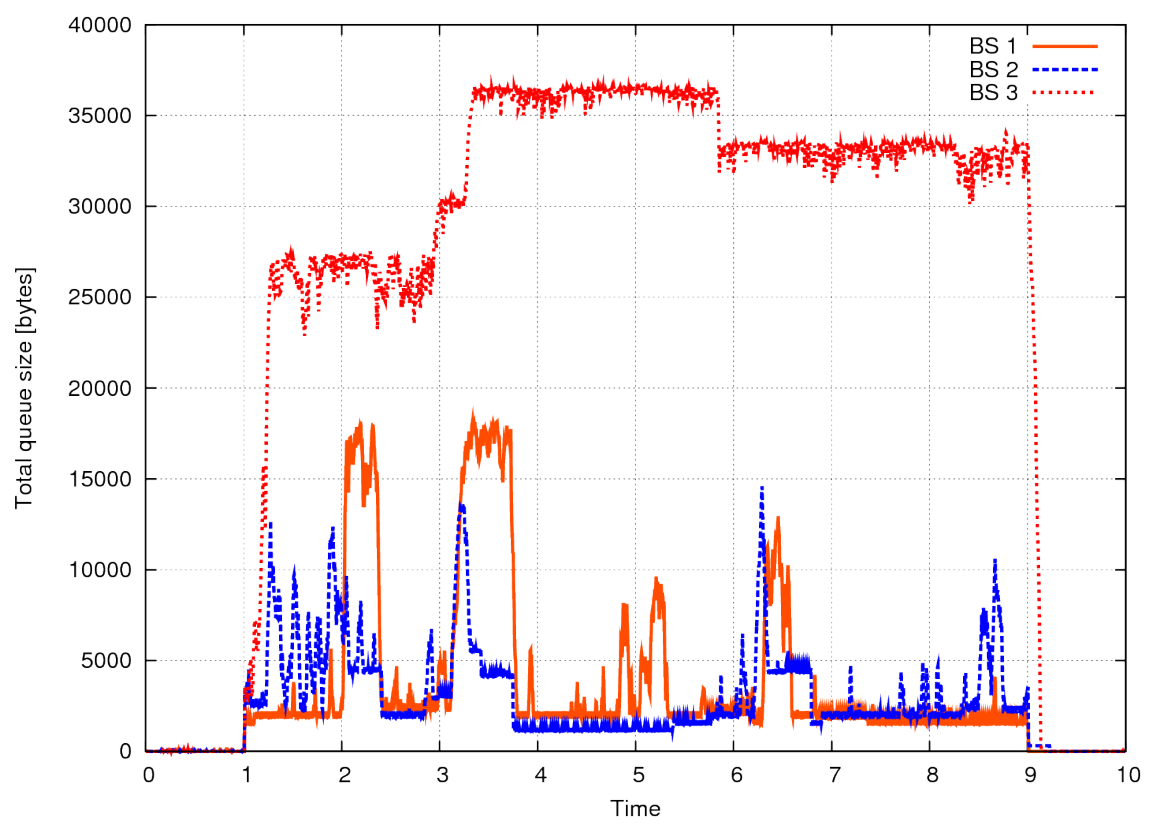

Figure 3.14: Downlink queue sizes at BSs.

The BS 3, which serves about half of the users during the whole simulation, understandably has the largest queue size, whereas the queues of the other BSs remain at reasonable levels. Peaks in the queue sizes of the BS 1 and BS 2 are likely caused by channel fluctuations resulting in increased packet retransmissions. Also handover and network re-entry signaling have small impact on queue size variations. All in all, cell edge users with constant traffic requirements might easily exceed the capacity of the serving BS due to enforced use of robust MCSs and big amount of packet retransmissions.

In figure 3.15 can be seen the outcome of insufficient handover triggering mechanisms resulting in overloaded BS. The figure shows in cumulative manner the number of packets that are dropped from the transmission queues of each of the BSs.

The number of queue drops start to increase at the BS 3 right after starting the UDP applications and continue growing until the applications are stopped at $9 \mathrm{~s}$. By looking at this figure it is obvious that the BS 3 cannot serve all the MSs simultaneously. Packet drops will cause prolonged delays and excessive retransmissions at the cell border cumber the BS even more.

The results here show that it is crucial to use other handover triggering mechanisms in addition to the RSSI measurement based mechanism. Furthermore, in 


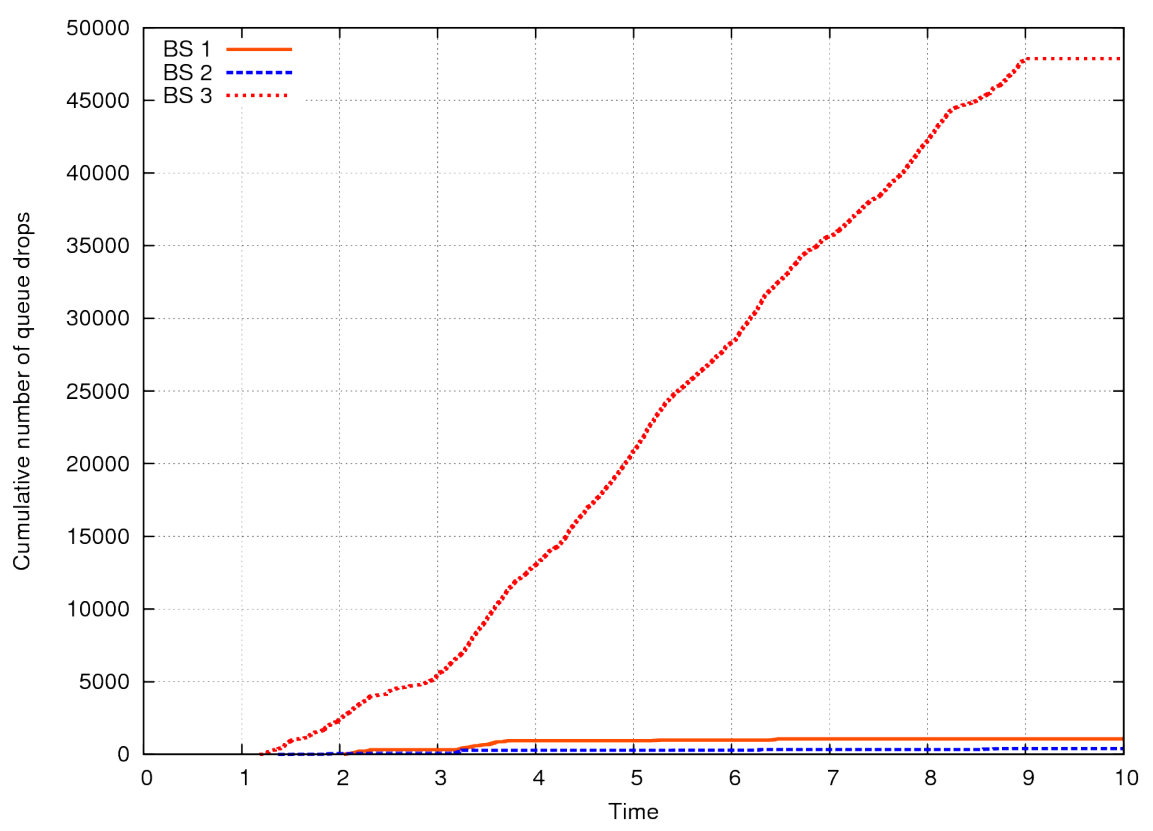

Figure 3.15: Cumulative amount of DL queue drops.

the example only 20 users were deployed. In reality a single BS may have to serve even hundreds of users. Taking into account the limitations in BSs' capabilities, a network controlled subscriber distribution over multiple BSs is recommended. 


\section{Further development ideas}

There exist many interesting fields of study related to mobility management and handovers. In this section some ideas for further development are brought forth. Although the following items relate closely to WiMAX, they can be applied also to other modern wireless network technologies, like HSPA and LTE. Thus, studying these topics does not have to be limited only to WiMAX.

A universal issue concerning all wireless technologies supporting mobility of users (or relay stations) is a design of handover initiation criteria. In this thesis only RSSI differences may trigger a handover. In reality there would be also other aspects that might result in a handover, such as load balancing or lack of capacity at serving BS. Supporting such sophisticated methods requires user and connection management at the access network, which the current simulator implementation lacks. Keeping in mind the simulation results which were shown earlier, it would be very beneficial to implement support for multiple handover triggering methods and algorithms. Using only single criteria, as in this thesis, it is possible that in some cases obtained results might not prove to be reliable, or at least they might not be realistic. It is also reasonable to concentrate on development of adaptive handover initiation algorithms that can be applied for various conditions and that can work in a coordinated way with the mobile access network.

The implementation of neighbor BS scanning is quite simple at the moment and does not follow the specification. Currently there is no attachment procedures nor different levels of association performed between a MS and neighbor BSs in order to allow signal strength scanning. Supporting neighbor advertisements and especially periodic scanning requests would require some basic association between BSs and MSs. Although, in network initiated handovers neighbor BS information could be collected quite easily by serving BS over access network.

Continuing the topic on the handover process development, fast ranging can be taken into use in WINSE. It optimizes network entry to new BS and accelerates the process by $5-10 \mathrm{~ms}$. This requires additional signaling between BSs which is not implemented at the moment. In general, access network can be exploited more in several operations, including handover and connection/service flow establishment.

Mobility in relay networks will be one of the most interesting topics under the field of mobility management, which companies developing wireless networks may 
be willing to invest in the near future. This may be the situation, especially, in HSPA networks, where the specification of multihop relays is under construction at the moment.

Expanding WiMAX studies to cover inter-ASN mobility and inter-BS forwarding over the R8 reference point are possible with current simulator implementation. This would be a topic very easy to start with, if one is interested in mobility management research. CSN-anchored mobility, although being as well a good topic for future, would need admission control and other core network functionality. Implementation of such features in WINSE and the ASN-GW module might not be trivial due to their current internal design. Taking into account the interest level of industry in WiMAX, many companies might consider heavy investments in research of CSN-anchored mobility, or mobility in WiMAX altogether, as a waste of resources. 


\section{Conclusions}

WiMAX is a network technology providing high speed Internet access to wireless users in LOS and NLOS conditions. The IEEE 802.16 specifications define air interface functionality between mobile station and base station, whereas the access network part is defined by WiMAX Forum's Network Working Group.

Full mobility support for moving subscribers in WiMAX network is provided by network management procedures between entities. Handover, where a MS transits from coverage area of one BS to coverage of another BS, is one of the essential features of mobility allowing users to maintain their data connections even while traveling at vehicular speeds. In order to reduce interruption times caused by handovers and to rapidly re-establish communication after handovers, optimization procedures are carried out between network elements over wireless channel and access network. ASN gateways in WiMAX control access network operations together with base stations. From amongst different mobility types, ASN-anchored mobility was examined, where mobile stations may move within coverage of one or more ASN-GWs, as long as their core network access point (CSN) remains the same.

Hard handover performance in the WiMAX network was studied in the thesis by means of simulations using the NS-2 network simulator. Impact of duration of the Time-to-trigger timer to the handover process was investigated. The goal was to provide, in some extent, preferable handover related parameter values. Obtained results revealed that there are remarkable variance in network behavior depending on the Time-to-trigger time which explicitly affects moment of time when handover is started. This may also change overall network performance.

As a conclusion, the handover initiation criteria should depend on channel conditions and surrounding environment. A longer Time-to-trigger suits well if slow user velocity is expected, as it will reduce the ping-pong effect efficiently. A short Time-to-trigger can be applicable in high velocity mobility. In such situation number of handovers remain at low level while the performed BS switchings are carried out at early stage of MS movement. This way wireless communication to old BS does not have time to deteriorate drastically before a handover. A smaller Time-to-trigger value can be a good choice for all the environments if large signaling load due to high amount of handovers can be accommodated by the access and core network.

The presented results demonstrate how it is necessary to implement another han- 
dover criteria in the network, for instance, for load balancing purposes between BSs. Without such user control at access and core network a single BSs may have to carry a significant burden by serving high proportion of users while other BSs are underloaded. From the results, it seems clear that inadequate or bad design of mobility management will result in decreased network performance. 


\section{References}

[1] IEEE, IEEE 802.16e-2005, IEEE Standard for Local and metropolitan area networks, Part 16: Air Interface for Fixed and Mobile Broadband Wireless Access Systems, Amendment 2: Physical and Medium Access Control Layers for Combined Fixed and Mobile Operation in Licensed Bands and Corrigendum 1, available in PDF format <URL: http: / / standards. ieee.org/getieee802/download/ $802.16 e-2005$. pdf>, February 28, 2006.

[2] IEEE, IEEE 802.16-2004, IEEE Standard for Local and Metropolitan area networks, Part 16: Air Interface for Fixed and Mobile Broadband Wireless Access Systems, available in PDF format <URL: http://standards. ieee.org/getieee802/download/ 802.16-2004.pdf>, October 1, 2004.

[3] IEEE, IEEE 802.16j/D7, Draft Amendment to IEEE Standard for Local and Metropolitan Area Networks, Part 16: Air Interface for Fixed and Mobile Broadband Wireless Access Systems, Multihop Relay Specification, October 20, 2008.

[4] WiMax.com Broadband Solutions, Inc., community portal for the worldwide WiMAX community, available in WWW format <URL: http: / /www.wimax.com>, referred on August 14, 2009.

[5] Conniq.com, Duplexing Scheme in WiMAX: TDD or FDD, available in WWW format <URL: http: //www. conniq.com/WiMAX/tdd-fdd.htm>, referred on August 20, 2009.

[6] WirelessMAN, IEEE Std 802.16j: Publication History, available in WWW format <URL: http: //wirelessman.org/pubs/80216j.html>, referred on August 20, 2009.

[7] WiMAX Forum, available in WWW format <URL: http: //www. wimaxforum. org>, referred on August 21, 2009.

[8] IEEE, IEEE P802.16Rev2/D5, Draft Standard for Local and metropolitan area networks Part 16: Air Interface for Broadband Wireless Access Systems, June, 2008. 
[9] H. Martikainen, O. Alanen and A. Sayenko, ARQ Parameters for VoIP in IEEE 802.16 networks, Wireless Telecommunication Symposium, Prague, Czech Republic, 2009.

[10] eTutorials.org, Free programming tutorials, Wimax Technology for broadband wireless access, Part Three: WiMAX Multiple Access (MAC Layer) and Qos Management, available in WWW format <URL: http://etutorials.org/Networking/wimax+technology+ broadband+wireless+ access/Part+Three+WiMAX+Multiple+ Access+MAC+Layer+and+Qos+Management/>, referred on August 24, 2009.

[11] Yan Zhang and Hsiao-Hwa Chen, Mobile WiMAX: toward broadband wireless metropolitan area networks, page 95, Auerbach Publications, 2008.

[12] The Internet Engineering Task Force (IETF), Internet home page, RFC pages, available in WWW format <URL: http://wWw. ietf.org/rfc.html>, referred on August 25, 2009.

[13] The Multiserver Forum, MSF Architecture for WiMAX Access Network, available in PDF format <URL: http: / / www.msforum.org/techinfo/approved/MSFR4-ARCH-WIMAXFINAL • pdf>, July 9, 2008.

[14] WiMAX Forum, Network Architecture (Stage 2: Architecture Tenets, Reference Model and Reference Points), Part 2, Release 1.5, Draft Version 1-C, March 6, 2009.

[15] WiMAX Forum, Network Architecture (Stage 3: Detailed Protocols and Procedures), Release 1.5, Draft Version 1-D, April 16, 2009.

[16] M. Majanen, P. H. J. Perälä, T. Casey, J. Nurmi and N. Veselinovic, Mobile WiMAX Handover Performance Evaluation, Fifth International Conference on Networking and Services, ICNS '09, Valencia, Spain, 2009.

[17] L. Chaari ja L. Kamoun, An Overview of Mobility Management Over IEEE802.16e, International Conference on Telecommunications, ICT '09, Marrakech, Morocco, 2009.

[18] WirelessLogix, E2E Network Solutions, MS-Initiated Hard Handover in WiMAX, available in PDF format <URL: http://www.wirelesslogix.com/customerportal/upload/WL_ WiMAX_MS-Initiated_HHO_WP_v1.pdf>,April 11, 2008. 
[19] M. Mendieta, Modeling of Test Specifics for Multi-vendor WiMAX Networks, Master's thesis, Mannheim University of Applied Science, October 30, 2008

[20] Z. Becvar and J. Zelenka, Handovers in the Mobile WiMAX, Research in Telecommunication Technology 2006, available in PDF format <URL: http://fireworks.intranet.gr/publications.asp>, referred on September 1, 2009.

[21] A. Mäkeläinen, Analysis of Handover Performance in Mobile WiMAX Networks, Master's thesis, Helsinki University of Technology, December 3, 2007.

[22] IEEE, IEEE Std. 802.21-2008, IEEE Standard for Local and metropolitan area networks, Part 21: Media Independent Handover services, available in PDF format <URL: http: / / standards. ieee.org/getieee802/ download/802.21-2008.pdf>, January 29, 2009.

[23] WiMAX Forum, WiMAX Roaming, Internet home page, available in WWW format <URL: http://www.wimaxroaming.org/>, referred on September 10, 2009.

[24] SearchMobileComputing.com, Roaming service, available in WWW format <URL: http: / / searchmobilecomputing.techtarget.com/ sDefinition/0,sid40_gci212911,00 .html>, October 20, 2008.

[25] A. Sayenko, O. Alanen, H. Martikainen, V. Tykhomyrov, A. Puchko and T. Hämäläinen, WINSE: WiMAX NS-2 Extension, 2nd International Conference on Simulations Tools and Techniques, SimuTools '09, Rome, Italy, 2009.

[26] USC/ISI, NSF, The Network Simulator - ns-2, available in WWW format <URL: http://www.isi.edu/nsnam/ns/>, referred on September 18, 2009.

[27] J. Karhula, Simulating WiMAX network with NS2 network simulator, Master's thesis, University of Jyväskylä, February 17, 2006.

[28] USC/ISI, NSF, The Network Simulator - ns-2, Trace file formats, available in WWW format <URL: http://nsnam.isi.edu/nsnam/index.php/NS-2_Trace_Formats>, July 1, 2009.

[29] Sourceforge, $\mathrm{TclCl}$, available in WWW format <URL: http://otcl-tclcl.sourceforge.net/tclcl/>, referred on September 20, 2009. 
[30] Telecom ABC, IMT-Advanced, available in WWW format <URL: http://www.telecomabc.com/i/imt-advanced.html>, referred on September 20, 2009.

[31] International Telecommunication Union, ITU global standard for international mobile telecommunications 'IMT-Advanced', available in WWW format <URL: http: / / www.itu.int/ITU-R/index.asp? category=information \& rlink=imt-advanced\&lang=en>, September 12, 2008.

[32] IEEE, A Generic Packet Convergence Sublayer(GPCS) for Supporting Multiple Protocols over 802.16 Air Interface, available in PDF format <URL: http: //www. ieee802.org/16/netman/contrib/ s80216g-05_025.pdf>, November 25, 2005.

[33] Radio-Electronics.com, handoff, available $\begin{array}{crr}\text { Cellular } & \text { Handover } & \text { and } \\ \text { in } & \text { WWW } & \text { format }\end{array}$ http: / / www.radio-electronics.com/info/cellulartelecomms/ cellular_concepts/handover_handoff.php>, referred on September 27, 2009.

[34] The IEEE 802.16 Working Group on Broadband Wireless Access Standards, IEEE 802.16 Published Standards and Drafts, available in WWW format <URL: http: //wirelessman.org/published.html>, referred on September 27, 2009.

[35] Mark C. Wood, An Analysis of the Design and Implementation of QoS over IEEE 802.16, available in WWW format <URL: http://www.cse.wustl.edu/ jain/cse574-06/ftp/wimax_qos/ index.html>, April 23, 2006.

[36] IEEE 802.16 Broadband Wireless Access Working Group, IEEE 802.16m Evaluation Methodology Document (EMD), available in PDF format <URL: http://www.ieee802.org/16/tgm/core.html\#08_004>, January 15, 2009. 
Appendices 


\title{
A Handover performance in the IEEE 802.16 mobile networks
}

\author{
By
}

V. Hytönen, A. Sayenko, H. Martikainen and O. Alanen 


\section{Handover performance in the IEEE 802.16 mobile networks}

\author{
Vesa Hytönen \\ Telecommunication Laboratory \\ University of Jyväskylä, \\ Finland \\ vesa.a.hytonen@jyu.fi
}

\author{
Alexander Sayenko \\ Research, Technology \& \\ Platforms, \\ Nokia Siemens Networks, \\ Espoo, Finland \\ alexander.sayenko@nsn.com \\ Henrik Martikainen \\ Telecommunication Laboratory \\ University of Jyväskylä, \\ Finland \\ henrik.martikainen@jyu.fi \\ Olli Alanen
Magister Solutions Ltd
Jyväskylä, Finland
olli.alanen@magister.fi
}

\begin{abstract}
The IEEE 802.16 standard provides a full mobility support for stations moving across cell borders. In addition, the WiMAX Forum Network Working Group defines a set of procedures to support handovers in mobile access networks. One of the main characteristics of mobility support is fully controlled and optimized handover ( $\mathrm{HO})$, in which a mobile station (MS) migrates from the air interface of one base station (BS) to the air interface of another BS. It is vital to keep the BS transition phase as short as possible to decrease delays and data loss, which is especially crucial for real-time applications, such as VoIP. In this paper we analyze ASNanchored mobility performance and provide advisable handover related parameters. The obtained results show that it is reasonable to adapt the handover initiation functionality to the environment where the network is deployed.
\end{abstract}

\section{Categories and Subject Descriptors}

C.2.3 [Computer-Communication Networks]: Network Operations

\section{Keywords}

ASN-anchored mobility, handover, IEEE 802.16 WiMAX, NS-2, WMF NWG

\section{INTRODUCTION}

IEEE 802.16 is a standard for the wireless broadband access network $[1,2,5]$ which can provide a high-speed wireless access to the Internet to home and business subscribers. Providing a reliable connection for subscribers over a wireless channel is a challenging task, which becomes even more difficult when subscribers are allowed to move across cell borders. It leads to situations where they need to change the point-of-attachment $(\mathrm{PoA})$ in the network in order to maintain a desirable level of communication. A basic PoA switching method defined in the IEEE 802.16 standard is called hard handover (HHO), in which the MS drops connections with the old BS or relay station (RS) before associating itself to a new BS or RS. This kind of break-beforemake transition always causes some period of unavailability during which the MS is unable to transmit or receive data. To reduce handover interruption times and increase the performance of the network, some optimization methods can be performed where utilization of the access network backhaul plays a major role.

A network re-entry process may be divided into a preparation phase and an action phase. In both stages, the WiMAX network backbone can be exploited to boost the HO operations. During the preparation phase the MS context and handover messages can be transferred between BSs over the access network. When the actual handover takes place, some parts of the necessary information will already be available for the new BS, and the information exchange at this stage can be partly omitted. As a result, the optimized handover procedures allow usage of real-time services with strict delay constraints [5].

Fluctuations in the received signal strength create challenges in choosing correct $\mathrm{HO}$ triggering criteria. Using badly designed criteria results in handovers being performed either too sparsely or too frequently, reducing in both cases the network performance. In this paper we examine the impact of Time-to-trigger timer to handover initiation and network statistics. The timer explicitly affects the moment of time when a handover process is started, therefore it provides a simple way to study network performance in different conditions where handovers are executed. The results shown later in this paper may be used for further development of HO initiation methods.

Handover related topics have been studied quite widely by several researchers. In [8], the authors focus on handover initiation algorithms in WCDMA taking into account the effect of hysteresis margin and the Time-to-trigger on handover initiation mechanism. They utilize soft handover and active set update which are not of interest for our study. Furthermore, the impact of handover initiation on overall 
HO progression is not investigated. The paper, nevertheless, provides a good basis for further study of handover initiation. In [9] hard handover in WiMAX is studied, but the paper does not account for the effect of Time-to-trigger duration to handover initiation. However, it offers a detailed description of the impact of association and optimization methods to handover performance. In [7], the authors consider predominantly theoretical aspects of mobility management in an 802.16 network, but they do not provide statistics or a simulation based outcome.

To the best of our knowledge, only few researches utilize access network implementation for their handover studies. This could be an important factor when considering the reliability of the simulations. In light of this, the results shown later in this paper provide very accurate handover related simulation statistics that can be used as basis for further study.

In this paper we consider the ASN-anchored mobility defined in [4] in which an MS may move within the coverage areas of base stations located in the same Access Service Network (ASN). This type of MS movement is called intra-ASN mobility. From the network element deployment point of view, it is reasonable to determine the areas within which people preferably move. The base stations in these areas are served usually by the same ASN node. Thus, in this paper we simulate intra-ASN mobility, which is the most common mobility type in WiMAX. The ASN-anchored mobility also includes inter-ASN mobility in which stations may move across multiple ASNs. However, this remains outside of the scope of the paper.

The rest of this paper is organized as follows. In Section 2 we introduce the reader to the handover process from wireless link and access network points of view. From there, we continue to introduce our simulations and results in Section 3. Section 4 concludes the paper.

\section{BASIC MOBILITY SUPPORT IN WIMAX}

This section concentrates on describing optimized hard handover in ASN-anchored mobility events. The handover process is depicted mostly from the IEEE 802.16 standard point of view, focusing on wireless communication. The ASNanchored mobility is defined by the WiMAX Forum's Network Working Group (NWG), thus the description of this mobility type introduces mostly access network functionality during HOs.

The WiMAX reference model depicted in Fig. 1 shows the logical composition of a WiMAX network. Network entities are connected by reference points designated as mediates for bearer plane or control plane functions. The ASN-anchored mobility exploits intra-ASN R6 reference point for communication between BS and ASN-GW. As shown in Fig. 2 an ASN consists at least of one BS and one ASN gateway (ASNGW), which are able to control traffic and load balance within one ASN or among multiple ASNs.

\subsection{Handover process}

A handover may be triggered by various reasons, each of which is usually aiming to provide the highest level of service available for the MS. In this paper, we hold to the Received

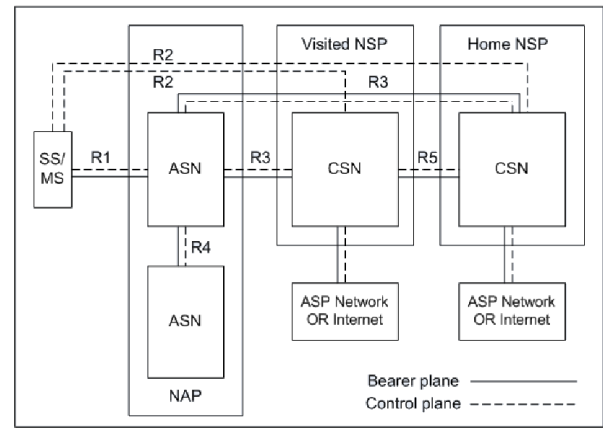

Figure 1: WiMAX network reference model.

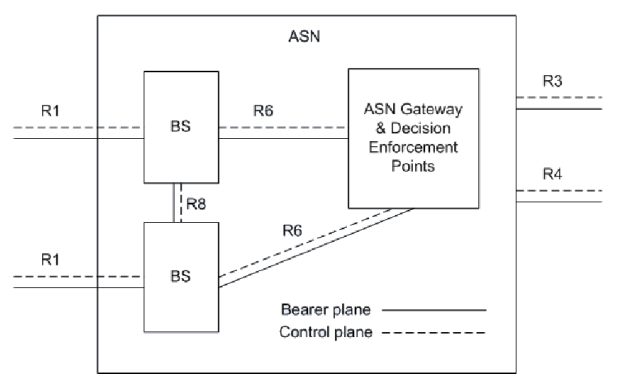

Figure 2: ASN decomposition.

Signal Strength Indication (RSSI) measurement as a handover triggering mechanism. With this method there exist at least two distinct variables which determine the handover process commencement, namely hysteresis margin $P_{\text {hyst }}$ and Time-to-trigger duration $T_{\text {trig }}$. Prior to the handover preparation phase the MS surveys signal strength levels during predetermined scanning intervals from the serving BS as well as from nearby BSs for RSSI comparisons. In a simple handover initiation algorithm the MS starts a Time-to-trigger timer if the signal strength from the neighboring BS $P_{n b r}$ exceeds the sum of $P_{\text {hyst }}$ and the signal strength of the serving BS $P_{s r v}$. If such condition remains through the whole $T_{t r i g}$ duration, the MS considers the neighbor BS in question to be a suitable target for migration. [5][9][7]

Once a decision to perform a $\mathrm{HO}$ is made, the handover process may be initiated either by the mobile or the network. As seen from Fig. 3(a) the handover preparation phase begins with the transmission of a handover request message that indicates a need for PoA switching; a MOB_MSHOREQ in mobile initiated handover and a MOB_BSHO-REQ in network initiated handover. A request message contains, for instance, identifiers of the selected target BS candidates. Upon reception of the MOB_MSHO-REQ the serving BS transmits a HO_Req message to target ASN over the backbone network. Particularly, MS's service flow information is stored in this message, which is required for the re-establishment of transport connections after the handover. [5][4]

After receiving and processing the handover request, the target ASN replies with a HO_Rsp message. When the serving BS receives the HO_Rsp it transmits a MOB_BSHO-RSP message to the MS over the air interface. The purpose of the MOB_BSHO-RSP is to report the MS about BSs that 
are ready to take the MS under their control. It may also tell the MS about a pre-allocated fast ranging time interval which can be used to accelerate the network entry after the HO. [5][4]

The MS decides the eventual target BS and adds its identifiers into a MOB_HO-IND message which the MS transmits to the serving BS. This will be the last message sent to the old BS before the actual HO, after which the MS terminates connections with the serving BS and starts its ranging operation with the target BS. Provided that the MS has accepted the candidate target $\mathrm{BS}$ to become its new PoA, upon reception of the MOB_HO-IND the serving BS will transmit a HO_Cnf message to the target ASN. After MS's successful network re-entry to the target BS (which becomes a new serving BS after the $\mathrm{HO}$ ) a data path registration process is started between the target ASN and the anchor ASN for proper functionality of the bearer plane functions. [5][4]

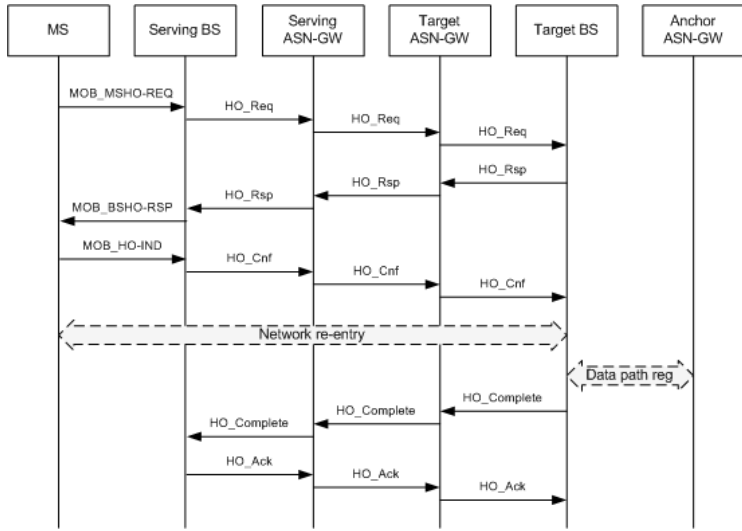

(a) Management messages during handover.

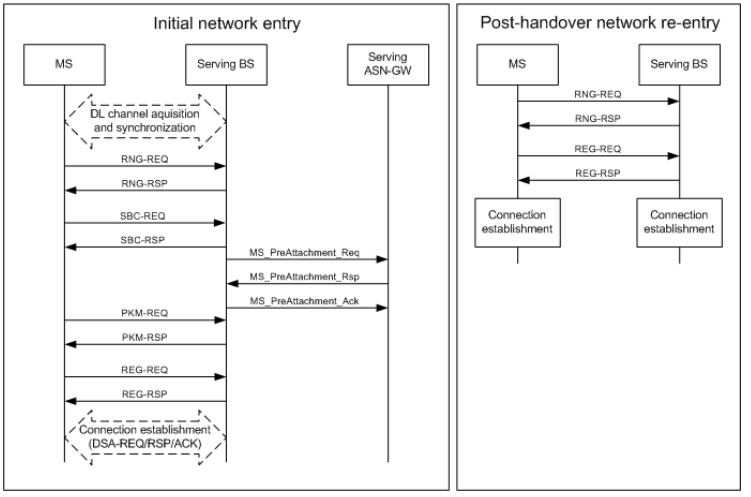

(b) Initial and post-handover network entry messages.

Figure 3: Management message exchanges.

During a handover some MS context may already be transferred to the target ASN over the backbone network. Furthermore, the target ASN may request for MS's context from the serving ASN over the backbone network in spite of having received the context earlier. This type of handover is called optimized handover and its task is to keep handover latencies and interruption times small, allowing usage of VoIP and other real-time applications. An MS performing an optimized handover may omit exchanging some information during the network re-entry. This includes, for example, exchange of SBC-REQ/RSP messages, some parts of PKM authentication and service flow encodings. [5][4]

\subsection{ASN-anchored mobility}

The ASN-anchored mobility is defined as mobility of an MS which does not contain change of Foreign Agent (FA) and Care-of-Address (CoA) points. As the R3 reference point is not utilized in the case of ASN-anchored mobility, the MS is allowed to migrate between BSs located in the same ASN or in different ASNs as long as it does not change the traffic anchor ASN and thus the Connectivity Service Network (CSN) through which the MS is connected to core network. ASN-anchored handover procedures include MS's context exchange between ASNs and BSs via the R4 and R6 reference points, packet loss reduction and maintaining packet ordering and latency. Intra-ASN mobility management (MM) and Inter-ASN MM are covered by the ASNanchored MM category. The ASN-anchored mobility specification defines three functions, namely Data Path (Bearer plane) function, Handover function and Context function. These take care of data path setup and packet transmissions, handover decision and signaling procedures and MS related context acquisition, respectively. [4]

The functions above reside at ASNs which can be divided into four groups during a HO process: anchor ASN, serving ASN, target ASN and authenticator ASN (Fig. 4) [4].

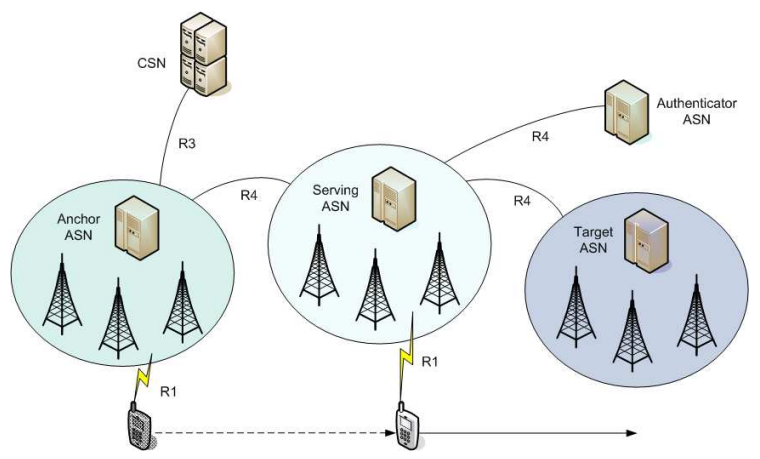

Figure 4: ASN-anchored mobility.

The anchor ASN operates as a connection point to the CSN and network services by hosting the anchor DP function for the MS. The serving ASN serves the MS prior to a $\mathrm{HO}$ and manages the serving $\mathrm{HO}$ function. The target ASN hosts the target $\mathrm{HO}$ function. It is the network entity the MS will be bound after a successful HO, thus becoming a new serving ASN. Authenticator ASN provides the authenticator/key distributor function for the MS. [4]

The [4] defines three profiles for the ASN which determine the decomposition of the functions and tasks within the BS and the ASN-GW. The network administrator may choose each of the profiles as a basis for physical implementation, after which other profile implementations can be excluded. Different profiles allow combining the BS and ASN-GW functions in the same network element or placing them into separate entities, making implementation of the access network flexible. Our implementation may be categorized in profile C, as the Radio Resource Control (RRC) and the Ser- 
vice Flow Management (SFM) reside at the BS. However, since not all the functionality included in the profiles is implemented in the simulator, it is not meaningful to strictly classify our simulator implementation in one specific profile.

\section{SIMULATIONS}

\subsection{Simulation environment}

We simulate handover operations with WINSE, which is an 802.16 extension to the NS-2 network simulator [10]. It introduces the $802.16 \mathrm{PHY}$ layer functionality, as well as a very detailed MAC layer implementation. To create realistic handover simulations, we also expand the simulator functionality with the ASN-GW module that enables access network modeling and ASN-anchored mobility in NS-2.

Fig. 5 shows the network topology used in our simulations. It contains three sectors from three different BS sites. Each BS sector applies a directional antenna covering 120 degrees, with the antenna angles pointing at 0, 120 and 240 degrees. The inter-site distance is 1000 meters. All the BSs are connected to the same ASN-GW via wired links. We use R4 and $\mathrm{R} 6$ reference points for communication between BSs and ASNs, so inter-BS forwarding over R8 is not utilized.

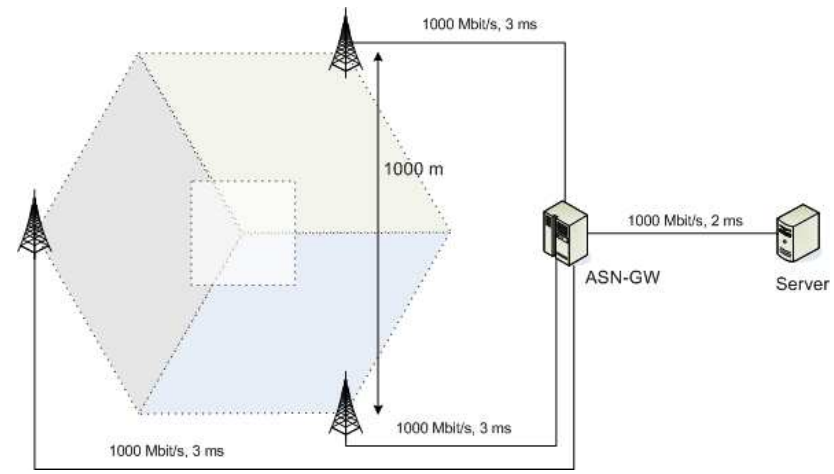

Figure 5: Network topology for the simulations.

In the simulations mobile stations are positioned randomly inside the rectangular area crossing the cell edges. The stations select a BS and perform a network entry according to their initial RSSI measurements from each BS. In the beginning of the simulation all the MSs start to move towards a randomly determined location within the rectangular area, where they might perform handovers between BSs. The [3] defines use cases for the HO performance evaluation. They propound MS trajectories covering movement across border of two cells as well as MS's movement along the border. For the sake of brevity, we do not use separate simulation scenarios designed for different trajectories, but rather utilize a single topology. By introducing random starting and destination points, we simulate various trajectories including the ones specified in the $802.16 \mathrm{~m}$ EMD document. We use mobile station velocities of $2 \mathrm{~m} / \mathrm{s}, 5 \mathrm{~m} / \mathrm{s}, 10 \mathrm{~m} / \mathrm{s}$ and $20 \mathrm{~m} / \mathrm{s}$ to study whether the movement speed impacts handover procedures. In addition to different MS velocities, we use four $T_{\text {trig }}$ values: $50 \mathrm{~ms}, 100 \mathrm{~ms}, 250 \mathrm{~ms}$ and $500 \mathrm{~ms}$. They affect, explicitly, the point of time when a HO is initiated and, implicitly, network performance statistics.

Table 1 shows the parameters we use in the simulations, which correspond to the WiMAX Forum system profile [6]. A constant hysteresis margin, $3 \mathrm{~dB}$, is used for handover initiation precondition.

Table 1: 802.16 network parameters.

\begin{tabular}{|l|l|}
\hline Parameter & Value \\
\hline Center frequency & $2.5 \mathrm{GHz}$ \\
Reuse factor & $1 / 3$ \\
Bandwidth & $10 \mathrm{MHz}$ \\
PHY & OFDMa \\
Duplexing mode & TDD \\
Frame duration & $5 \mathrm{~ms}$ \\
Channel report type / interval & CQICH / 20ms \\
Channel measurements DL/UL & preamble / data burst \\
Channel measurements filter & EWMA, $\alpha=0.25$ \\
Link adaptation model & target FEC BLER, 10 \\
\hline Antenna technique & SISO \\
BS / SS Tx power & $10 / 0.25 \mathrm{~W}$ \\
BS / SS antenna pattern & $3 \mathrm{GPP} /$ omni \\
BS / SS antenna height & $32 / 1.5 \mathrm{~m}$ \\
Inter-station distance & $1000 \mathrm{~m}$ \\
path loss & $.16 \mathrm{~m} \mathrm{Ua}$ \\
fast fading K factor & $0 \mathrm{~dB}$ \\
handover hysteresis margin & $3 \mathrm{~dB}$ \\
time-to-trigger & $0.05,0.1,0.25,0.5 \mathrm{~s}$ \\
\hline HARQ & Type I $(\mathrm{CC})$ \\
HARQ channels & 16 \\
HARQ buffer size & $2048 \mathrm{~B}$ (per channel) \\
HARQ shared buffer & ON \\
HARQ max. retransmissions & 4 \\
HARQ ACK delay & $1 \mathrm{frame}$ \\
PDU SN & ON \\
PDU SN type & long $(2 \mathrm{bytes})$ \\
\hline Ranging transm. opport. & 2 \\
Ranging backoff start/end & $1 / 15$ \\
Request transm. opport. & 2 \\
Request backoff start/end & $0 / 15$ \\
\hline PDU size & Unlimited \\
Packing/Fragmentation & ON \\
&
\end{tabular}

In addition, Table 2 presents the retransmission timeout values that we use in our simulations. These are very crucial for understanding the handover performance. If a certain management message is lost, the timeout value will trigger a message retransmission process.

Table 2: Retransmission times for management messages.

\begin{tabular}{|l|c|}
\hline Management message & Retransmission timeout [ms] \\
\hline RNG-REQ & 10 \\
SBC-REQ & 50 \\
PKM-REQ & 50 \\
REG-REQ & 10 \\
DSA-REQ & 200 \\
DSA-RSP & 100 \\
MOB_MSHO-REQ & 30 \\
\hline
\end{tabular}

Simulations with different velocities are conducted in a way where the simulation time depends on the velocity. We use a basic simulation time of 300 seconds which is divided by the velocity. As a result, during each simulation run an MS moves the same distance regardless of its velocity. This type of approach makes simpler a comparison of handover functionality with varying velocity when compared to a constant simulation time for all the velocities.

We use twelve simulation runs with different seed values for each combination of velocity and $T_{\text {trig }}$ for gathering enough information on the network behavior. In total, 20 stations are deployed in the simulations. Best effort UDP traffic is created in UL and DL for each user with the traffic transfer 
rate of $100000 \mathrm{bit} / \mathrm{s}$.

\subsection{Simulation results}

Keeping in mind that the SBC, PKM, and DSA exchanges are omitted in case of post-handover network re-entry as well as connection creation, the handover times can be easily kept below the initial network entry times. Fig. 6 shows CDF graphs for network entry times, including the first network entry that the MS performs and also the network entries that are done after failed HOs.

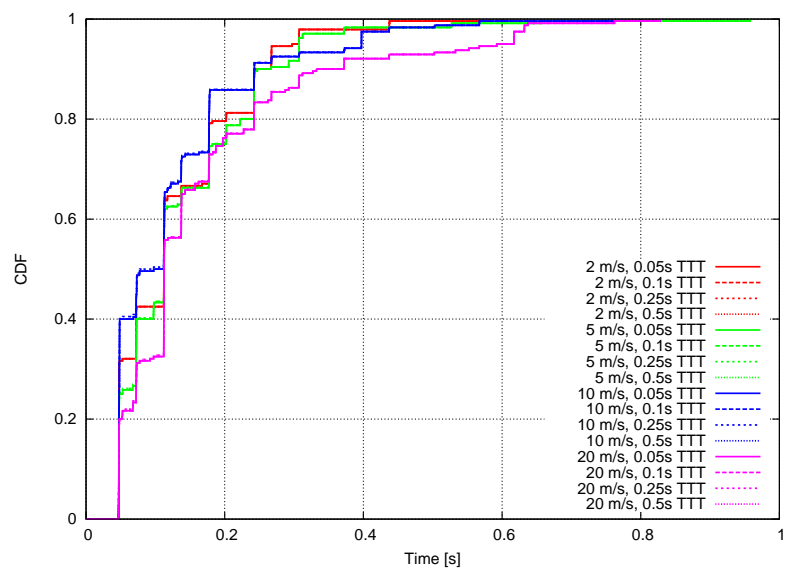

Figure 6: Initial network entry durations.

The steps in the graphs come from management message retransmissions and processing time. The $T_{t r i g}$ (TTT in the figure legend) does not affect the entry process itself, but rather affects the handover initiation moment and consequently the post-handover network entry. As the figure does not show statistics on network entries after successful HOs, here the $T_{\text {trig }}$ creates variance to the graphs only in case of a failed handover. The obtained results are useful for understanding the performance of the initial network entry process during a mobile equipment startup. However, such delays taking place during normal operation after failed handovers would cause significant performance degradation, being perceivable particularly with real-time traffic. ${ }^{1}$

Fig. 7 shows the number of successful and failed handovers performed during the simulations. In total, there were 2088 handovers and five of them failed, so the handover failure rate is approximately $0.24 \%$. As expected, the total number of HOs decreases with a longer $T_{\text {trig }}$ value and there might be simulation runs that do not have any handovers. An explanation for a larger number of HOs with $2 \mathrm{~m} / \mathrm{s}$ velocity and $0.05 \mathrm{~s} T_{\text {trig }}$ arises from the fast fading behavior. Fig. 8 shows RSSI levels from two BSs measured by an MS moving at the speed of $2 \mathrm{~m} / \mathrm{s}$. It can be observed that occasionally the fast fading does not vary fast enough thus resulting in a situation where the channel measurement and the link adaptation can follow channel variations. It is obvious that such a small $T_{\text {trig }}$ would not be suitable for small subscriber velocities which would usually be the case, for instance, in

${ }^{1}$ It is anticipated that the initial network entry time will be even larger because we did not simulate the process of a subscriber authentication by the mobile access network. Only the exchange of the PKM messages was modelled.

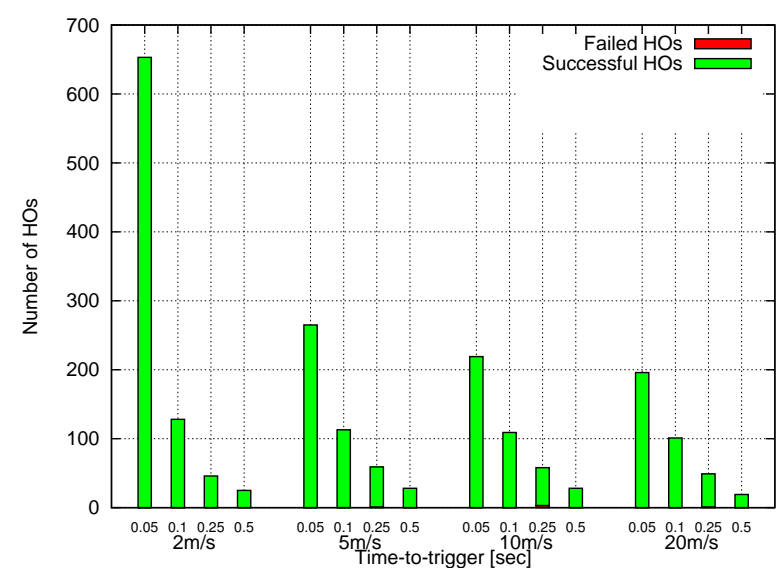

Figure 7: Total number of handovers.

city centrums or shopping malls. With higher velocities fast fading alters more rapidly where the channel measurement process just follows an average. Therefore, HOs do not occur so often even with a small $T_{\text {trig }}$.

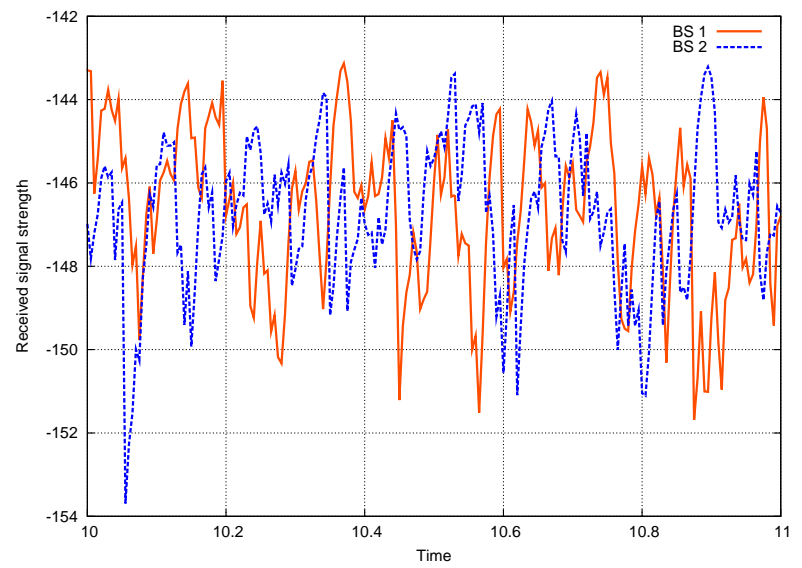

Figure 8: Impact of fast fading on RSSI levels.

A BS switching is preceded by a handover preparation phase starting from transmission of the MOB_MSHO-REQ message. The preparation is concluded with a MOB_HO-IND transmission, after which the MS deletes old connections. New connections are established after the network re-entry and registration to the target BS. Therefore the time gap between the MOB_HO-IND and the REG-RSP messages (handover interruption time) has a direct impact on packet delays and throughput. Durations of HO preparation ${ }^{2}$ and interruption $^{3}$ times are depicted in Fig. 9 and Fig. 10, respectively.

It can be seen from Fig. 9 that there is no relevant correlation between a particular $T_{\text {trig }}$ value and handover preparation time. It is rather the MS's velocity that impacts the

${ }^{2}$ Handover preparation time is measured from transmission of the MOB_MSHO-REQ to transmission of the MOB_HOIND message.

${ }^{3}$ Handover interruption duration is measured from transmission of the MOB_HO-IND to transmission of the REG-RSP. 


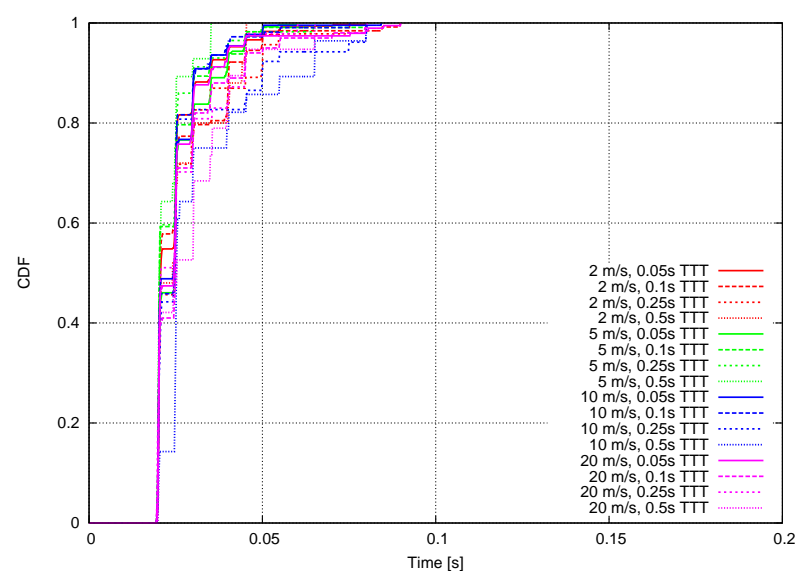

Figure 9: Handover preparation time.

rapidness of the handover preparation process. Indeed, the time needed for the handover handshake process depends merely on a fact that the handover messages are transmitted successfully, which, in turn, depends on the MS speed and other mechanisms, such as channel measurements, link adaptation, scheduling etc. Although the preparation times are quite small and do not interrupt a data transmission process, there seems to be lots of message retransmissions triggered either by the retransmission timers or the HARQ. This will increase overhead that, at the cell edge, will consume a large portion of the available bandwidth even without handovers. At least UDP traffic with even moderate data rate demand will suffer at this stage from excessive signaling.

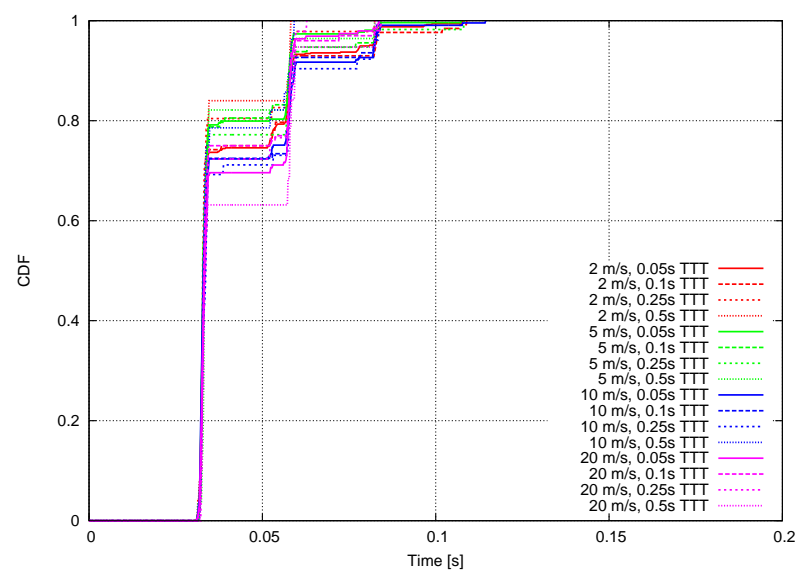

Figure 10: Handover interruption times.

Similarly to the handover preparation, the handover interruption times in Fig. 10 do not correlate with different $T_{\text {trig }}$ values. The Interruption time does not exceed 0.12 seconds and at least $60 \%$ of the post-handover network entries can be performed approximately within 30 milliseconds. With VoIP, such a disruption time should not decrease the quality of the connection excessively. For an explanation of the durations in the figure mere knowledge of retransmission timeout values is not enough, a detailed inspection of the network entry signaling is needed also. Fig. 11 shows two examples of network entry related message exchanges after a handover

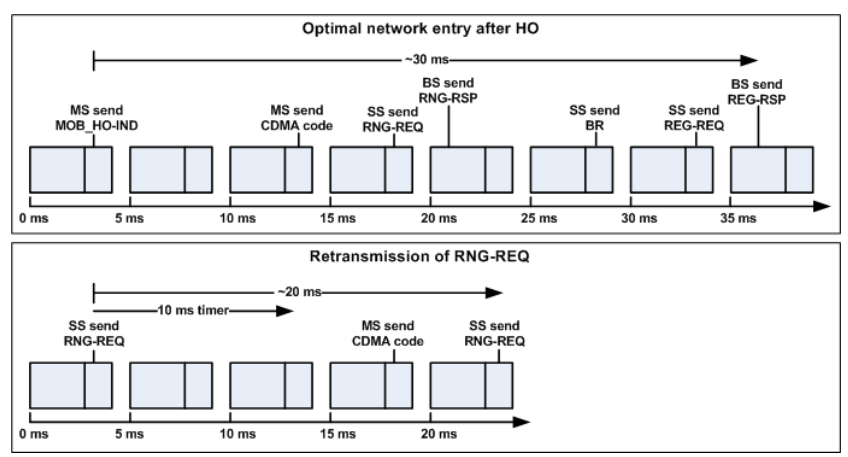

Figure 11: Network entry signaling during handover.

The procedure in the upper figure shows an optimal interruption time, that is, the entry process without message drops. From this figure it is clear that the interruption time is always at least $30 \mathrm{~ms}$. In the lower figure the RNG-REQ message is retransmitted once, increasing the network entry and interruption times by approximately $20 \mathrm{~ms}$. This is applicable also to subsequent retransmissions.

Total handover time, which is the sum of durations shown in Fig. 9 and Fig. 10, could be reduced by utilizing bandwidth pre-allocation mechanisms for HO messages. In addition, interruption times could be decreased with fast ranging, which was not applied in the simulations. With fast ranging, it is possible to hasten the re-entry process at least by $5 \mathrm{~ms}$ by pre-allocating bandwidth for the ranging request. Nevertheless, a clear advantage of the optimized handover to network entry durations can be seen when comparing initial network entry durations in Fig. 6 to re-entry times after handovers in Fig. 10. Durations in both figures are quite comparable since the measurements for Fig. 6 begin from the transmission of RNG-REQ and for Fig. 10 from the transmission of MOB_HO-IND, which is immediately followed by a transmission of the RNG-REQ message.

So far the findings have been focusing on the handover process itself. The impact of different $T_{\text {trig }}$ values to overall network activity may also vary considerably. In Fig. 12 we see the distribution of the Modulation and Coding Schemes (MCS) used for transmitted bursts when stations move at $20 \mathrm{~m} / \mathrm{s}$ velocity. For lower speeds similar behavior occurs, thus they are not shown here for the sake of brevity. In the simulator, 64QAM, 16QAM and QPSK modulations and coding rates of $5 / 6,3 / 4,2 / 3$ and $1 / 2$ are utilized. Furthermore, QPSK1/2 may use the repetition factors of 2,4 or 6. The most efficient MCS, 64QAM5/6, is located to the leftmost side of the figure and the robustness of the MCSs grow towards the right side, QPSK1/2 with the repetition factor of 6 being the most robust.

The $T_{\text {trig }}$ value of $0.05 \mathrm{~s}$ provides highest proportion of bursts that are able to use 16QAM MCSs which seem to be the most efficient MCSs available for the cell edge users in our simulation scenario. Better results for the smaller $T_{\text {trig }}$ values can be explained partly when we take into account the time the handover is performed. With $0.5 \mathrm{~s} T_{\text {trig }}$ 


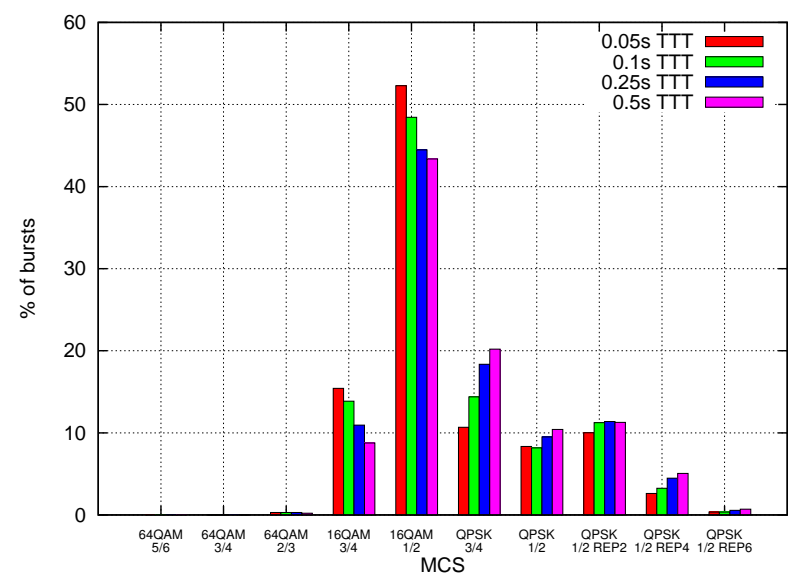

Figure 12: Distribution of MCSs at $20 \mathrm{~m} / \mathrm{s}$ MS velocity.

an MS may need to be connected to the old BS even several seconds longer than with $0.05 \mathrm{~s} T_{\text {trig }}$ before being able to make a handover to the new BS that provides a better channel. In our simulations there were differences of more than seven seconds in this. It is obvious that during this time an MS using a small $T_{\text {trig }}$ and migrating to better channel fast is able to utilize a higher amount of more efficient MCSs for its transmissions. Meanwhile a user with a longer $T_{\text {trig }}$ has to communicate through the old BS over a worse channel, thus being forced to use more robust MCSs.

The HO initiation time is not the only reason for such MCS distribution. As mentioned before, with a short $T_{\text {trig }}$ the RSSI measurement is able to follow the channel fluctuation caused by fast fading. This applies especially in small velocities. In such a situation the MS can migrate rapidly between BSs multiple times to acquire a better channel. On the other hand, one needs to remember the amount of connection drops with a small $T_{\text {trig }}$ due to numerous handovers. Packet drops are not visible in Fig. 12.

\section{CONCLUSION}

In this paper, we have analyzed the hard handover performance in the WiMAX network and the impact of the Timeto-trigger duration to the handover process. The results show that there are significant differences in network behavior depending on the handover initiation criteria. This might affect not only the handover process, but also the overall network performance.

Based on the obtained results the handover triggering criteria selection should depend on the environment where the network is deployed. It is reasonable to use larger Time-totrigger values to cater for slow user velocity environments in order to decrease the number of ping-pong effects. Furthermore, a slow speed will not result in drastic changes in shadow fading, which allows a user to stay for a longer period of time in its serving cell. In a high velocity environment, a smaller Time-to-trigger value is more suitable. The channel measurements and reporting, rather than following the fast fading, in this case follow slow fading. As a result, the amount of redundant handovers remains accept- able while the migration between BSs can be performed at an early stage of movement from one sector to another. Such a condition would be desirable, for instance, when moving with a car or train. In any case, a smaller Time-to-trigger value can be a valid choice for all the environments if the core network can accommodate a larger signaling load for the handover events.

There exist many interesting fields of research related to mobility management. Handovers in relay networks, in particular, have not been studied extensively. The research topic of this paper could be extended to cover inter-ASN mobility and CSN-anchored mobility. In light of all these concerns, one intriguing future topic would be development of adaptive handover initiation algorithm that can be applicable for various environments and channel conditions and can work in a coordinated way with the mobile access network.

\section{ACKNOWLEDGEMENTS}

We express our acknowledgments to Oleksandr Puchko and Vitaliy Tykhomyrov (University of Jyväskylä) for their contribution to the implementation of the 802.16 extension in the NS-2 simulator. Our special thanks to Le Yanqun (Nokia) for the initial implementation of the ASN-GW module and Milton Mendieta for the ASN-GW contributions. We would also like to thank Michael Gundlach (Nokia Siemens Networks) for providing a valuable insight on the functioning of the WiMAX mobile access network.

\section{REFERENCES}

[1] Air interface for fixed broadband wireless access systems. IEEE Standard 802.16, Jun 2004.

[2] Air interface for fixed broadband wireless access systems - amendment for physical and medium access control layers for combined fixed and mobile operation in licensed bands. IEEE Standard 802.16e, Dec 2005.

[3] IEEE $802.16 \mathrm{~m}$ evaluation methodology document (EMD). IEEE 802.16 Broadband Wireless Access Group, Mar 2008.

[4] WiMAX Forum Network Architecture, Sep 2008. Release 1, Version 1.3.0.

[5] Air interface for broadband wireless access systems. IEEE Standard 802.16 (Rev2), May 2009.

[6] WiMAX Forum Mobile System Profile Specification: Release 1.5 Approved Specification, Aug 2009.

[7] L. Chaari and L. Kamoun. An Overview of Mobility Management Over IEEE802.16e. In International Conference on Telecommunications, pages 334-339, May 2009.

[8] D. Kim and J. Kim. The optimum parameter design for wcdma intra-frequency handover initiation. In Networking and Mobile Computing, pages 239-248, Sep 2005.

[9] M. Majanen, P. H. J. Perälä, T. Casey, J. Nurmi, and N. Veselinovic. Mobile WiMAX Handover Performance Evaluation. In International Conference on Networking and Services, pages 263-269, April 2009.

[10] A. Sayenko, O. Alanen, H. Martikainen, V. Tykhomyrov, O. Puchko, and T. Hämäläinen. WINSE: WiMAX NS-2 Extension. In 2nd International Conference on Simulations Tools and Techniques, Mar 2009. 


\title{
B WINSE: WiMAX NS-2 Extension
}

\author{
By
}

\begin{abstract}
A. Sayenko, O. Alanen, H. Martikainen, V. Tykhomyrov, O. Puchko, V. Hytönen and T. Hämäläinen
\end{abstract}




\section{WINSE: WiMAX NS-2 Extension}

\author{
A. Sayenko \\ Research, Technology \& Platforms \\ Nokia Siemens Networks \\ Espoo, Finland \\ alexander.sayenko@nsn.com
}

\author{
O. Alanen, H. Martikainen, V. Tykhomyrov, \\ O. Puchko, V. Hytönen, T. Hämäläinen \\ Telecommunication laboratory, MIT department \\ University of Jyväskylä \\ Finland \\ \{olli.alanen,henrik.martikainen,vitykhom, \\ olpuehko,veashyto,timoh\}@jyu.fi
}

\begin{abstract}
IEEE 802.16 standard defines the wireless broadband technology called WiMAX. When compared to other wireless technologies, it introduces many interesting advantages at PHY, MAC, and QoS layers. Heavy simulations are needed to study IEEE 802.16 performance and propose further enhancements to this standard. Link level simulations are not always sufficient, while system level simulators are not always accurate to capture MAC and transport protocol details. We implemented a 802.16 extension for the NS-2 network simulator. It includes upper PHY modeling, almost all the features of the 802.16 MAC layer, as well as the QoS framework. This article describes the implemented features, simulation methodology, and shares our experience that can be used with other NS-2 modules. An overview of the past and ongoing research is given.
\end{abstract}

Keywords: IEEE 802.16 WiMAX, NS-2

\section{INTRODUCTION}

IEEE 802.16, called WiMAX, is a standard for the wireless broadband access network [2, 3] that can provide a highspeed wireless access to home and business subscribers. It can ensure diverse Quality-of-Service (QoS) requirements which makes IEEE 802.16 a scalable platform for many services [29]. The core components of a 802.16 system are a subscriber station (SS) and a base station (BS). The BS and one or more SSs can form a cell with a point-to-multipoint (PMP) structure. In this case, the BS controls the activity within a cell, resource allocations to achieve QoS and admission based on the network security mechanisms.

As any other wireless technology, IEEE 802.16 emerged from enormous number of technical contributions from various vendors. This technology continues to evolve through technical corrections, best practices, and more radical proposals. All these changes are supported by heavy simulations performed by researchers and engineers at various architectural levels. It is quite difficult, or even impossible, to accept a contribution if it is not based on reliable simulation results.

Traditionally, wireless network simulations are done with two different types of simulators. The first type of them is the link level simulators, in which a link between a base station and a subscriber station is modeled with a great level of details. The output from these simulators is usually bit, block and packet error probability under different parameters and conditions.

The second large group of simulators, which is widely adopted by industry, is the system level simulators. They model a number of geographical cells where base stations provide service to subscriber stations. Usually, the system simulators do not model all the link level aspects, but rather rely on the results obtained from the link level simulations. Nevertheless, their level of details include sub-carriers and a particular permutation type. System simulators are classified further into static and dynamic ones. Static simulators assume that subscriber stations do not move and the whole system is modeled with several "snapshots" of time, where stations' positions are randomly distributed over the simulation area. In the dynamic system simulations, stations can move over the simulation area performing network entries and handovers between the base stations. Therefore, the dynamic system simulators capture simulation results as a function of time. Their only disadvantage is significant complexity and a long simulation running time.

Third option for wireless simulations is the packet level simulators, such as NS-2 [47]. When compared to the dynamic system simulators, packet level simulators are very similar in terms of provided features. However, protocol stacks and application behavior are modeled more accurately. Similarly to the dynamic simulators, they account for many PHY aspects through abstractions and interfaces to link level simulators. The packet level simulators also allow for simulating the access service networks because it is possible to define a network topology where base stations, routers, gateways, and clients send or receive data. This makes it possible to obtain true end-to-end simulation results.

The rest of this paper is organized as follows. Section 2 gives a brief overview of other NS-2 802.16 modules. Section 3 provides details of our 802.16 extension. We do not delve into specific implementation details, but rather elaborate on simulation methodology and trade-off between complexity and simulation time. Section 4 gives an overview of 
past topics studied with our 802.16 module, while section 5 presents an overview of ongoing research. Finally, section 6 concludes the paper.

\section{PREVIOUS WORK}

In this section we give a brief overview of existent 802.16 modules for NS-2 that we are aware of. We refrain from any evaluation - our aim is to provide an insight on typical features that other wireless broadband packet-level simulators have or do not support. A more practical comparison and analysis of different 802.16 modules can be found in [15].

\subsection{NIST module}

NIST module is definitely one of the first 802.16 extensions for the NS-2 simulator. Even though the project goal aimed merely at studying the inter-network handovers, its result was also 802.16 MAC, handover, and scheduling extensions. The list of supported features as well as the general description is given in [37]. As a small summary, there is almost no proper PHY with a correct error generation. There is a OFDM PHY emulation, whereas WiMAX is based on OFDMa PHY. Absence of the ARQ mechanism makes it complicated to deploy the error model. The MAC level lacks several important features, such as packing.

\subsection{NDSL module}

This module is a result of a joint work between Chan Gung University and Institute for Information Industry. The module description and features are presented in [16]. Somewhat similarly to the NIST module, it focuses mostly MAC leaving the PHY level unattended. However, the MAC level operational parameters correspond to the OFDMa PHY. In addition, the MAC level implementation includes both fragmentation and packing; special attention to the management messages and network entry procedure is given. ARQ and HARQ retransmission mechanisms are not implemented. Despite a good set of features, further development of this module has stopped.

\subsection{WiMAX Forum module}

The WiMAX Forum has been developing its NS-2 802.16 extension that emerged from the NIST module. Since this module is available only for the WiMAX Forum members, only a brief overview will be given. Unlike a module from NIST, it focuses OFDMa PHY and tends to emulate the PHY behavior at the sub-carrier level. However, several important PHY features, such as channel reporting and link adaptation, were not introduced. The MAC level lacks a full support for ARQ; the HARQ retransmission is not implemented at all. Unfortunately, the WiMAX Forum announced that the development process will stop and the future of this module is not clear. Nevertheless, this module is very important in a sense that this was one of the first attempts to introduce PHY at the sub-carrier level granularity.

\subsection{Other modules}

There is a 802.16 extension for NS-2 from the Eurecom Institute. This is a relatively new module, features of which are described in [33]. Even though authors present it as a novel module with integrated QoS architecture, it does not differ in principle from the NIST implementation. The PHY model is not revised at all and the MAC timing works in accordance with OFDM PHY parameters.

There is also a module from KAIST university [23] and 802.16 extension for the NS-2 MIRACLE framework [13].

\subsection{Pisa university 802.16d Mesh module}

This module implements 802.16 Mesh that is an alternative to PMP mode. However, IEEE 802.16 working group discontinued 802.16 Mesh mode that is removed completely from the IEEE 802.16 evolution [6]. Absence of a standardization and industry support makes it quite complicated to compete with other ad-hoc technologies, such as 802.11s.

\section{WINSE}

\subsection{Overview}

WINSE is a WiMAX extensions for the NS-2 simulator. It was started as a small student project and then evolved into a powerfull simulation tool that now several companies use to study the MAC and QoS in the 802.16 system. Table 1 gives a short overview of features supported in WINSE.

\subsection{Core principles}

Before delving into the technical details, it is worth mentioning core principles behind our 802.16 module.

- Preserving existent framework. While introducing the 802.16 extensions, we do not change the NS-2 core classes, but rather introduce functionality by creating new ones. As considered in many papers, the NS- 2 core lacks many features and sometime suffers from a bad internal design. However, our approach is that researchers should be able to combine different modules on a common simulation platform to study more complex scenarios. Radical changes should be addressed by major revisions, e.g., NS-3.

- $\mathrm{C}++$ modularity. Emerged implicitly from the previous principle, there should be a class hierarchy with well defined class responsibilities. Fig. 1 shows the UML diagram with the top-level classes that constitute a core of our 802.16 module. Each functional block is contained in an independent $\mathrm{C}++$ class that allows for further virtualization and abstraction. 
Table 1. Features supported by WINSE.

\begin{tabular}{|l|}
\hline PHY \\
\hline OFDM and OFDMa PHY \\
HARQ: Type I, UL ACK channel \\
Channel reports: REP-RSP and CQICH \\
Link adaptation \\
\hline \hline MAC \\
\hline 802.16 d/e/j \\
Duplexing modes: TDD, FDD, H-FDD \\
DL broadcast messages: DL-MAP, UL-MAP, DCD, UCD \\
Compressed MAP, sub-MAPs \\
Connections: DL broadcast, basic management, transport \\
PDU construction, fragmentation, packing \\
Bandwidth requests: standalone \& piggy-backed \\
ARQ: blocks, feedbacks, timers, transmission window \\
Uplink contention: OFDM and CDMA-based for OFDMa \\
Network entry \\
Handover: SS-initiated, automatic \& manual \\
Sleep mode: class I, II, and III \\
\hline \hline QoS \& scheduling \\
\hline UGS, ertPS, rtPS, nrtPS, BE \\
BS scheduler \\
SS uplink scheduler \\
\hline \hline Access service network \\
\hline ASN-GW \\
R4, R6, and R8 interfaces \\
ASN-anchored mobility \\
\hline
\end{tabular}

- OTcl modularity. The most critical and fundamental $\mathrm{C}++$ classes are mapped to the correspondent OTcl classes. As can be seen from Fig. 1, all the major classes are derived, either explicitly or implicitly, from TclObject. It allows a script designer to change easily parameters and switch between different modules just by selecting their OTcl class names.

- Balance between the PHY and MAC features. The power of the NS-2 simulator is in transport and application level. It is not reasonable to go into the deep PHY modeling as it will increase significantly the computational burden. We select carefully PHY features to model and ways to model them to ensure that NS-2 does not turn into the link-level simulator.

\subsection{PHY Layer}

\subsubsection{PHY abstraction}

A properly designed PHY level must introduce an abstraction general enough to hide particular PHY details from other architectural components, such as MAC and scheduler. All the 802.16 modules mentioned in section 2 have a PHY

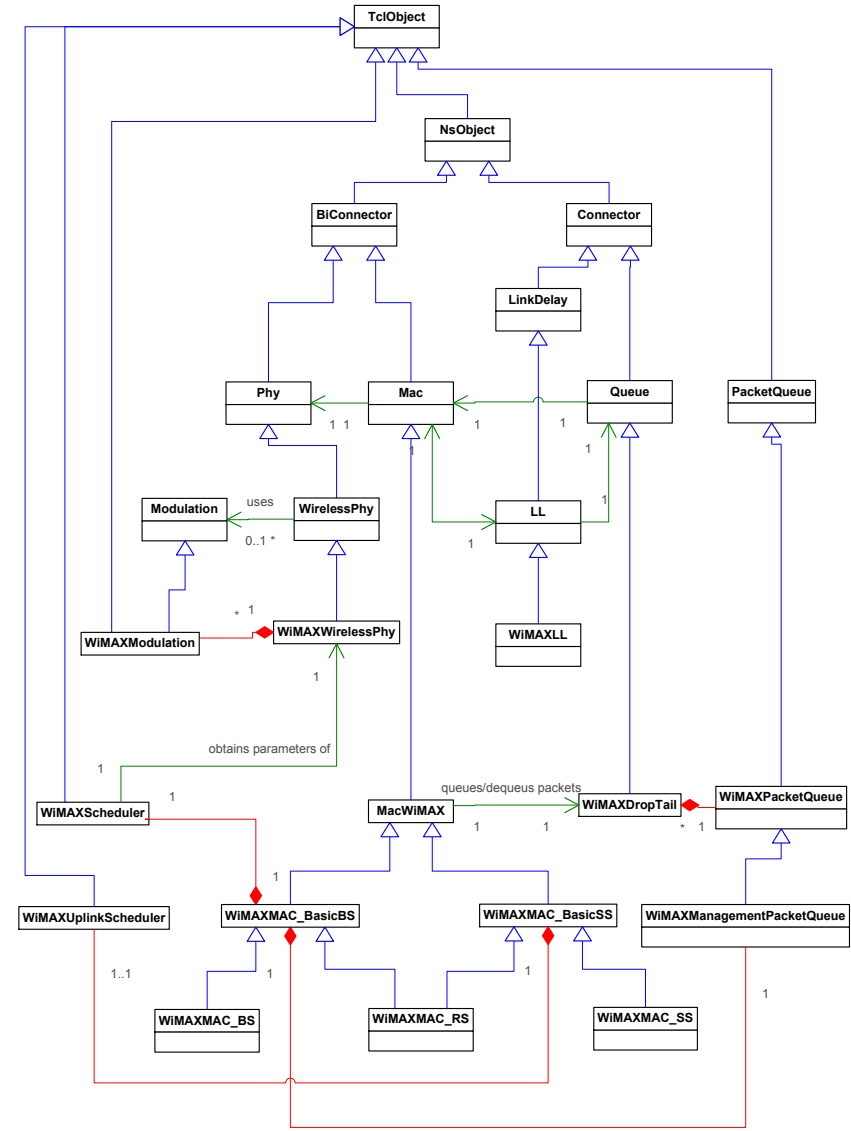

Figure 1. UML diagram for WINSE core classes.

specific MAC implementation resulting in either OFDM or OFDMa 802.16 simulator. Even though the industry chose OFDMa PHY as a basis for WiMAX networks, there are a lot of OFDM devices. Furthermore, an upcoming $802.16 \mathrm{~m}$ PHY also dictates a need in supporting simultaneously several PHYs.

Based on our experience, it is possible to provide such an abstraction if PHY exposes at least the following parameters:

- duplexing mode

- OFDM symbol duration

- zone parameters (number of OFDM symbols in a single slot and number of channels in one symbol)

- TTG and RTG gaps

- effective slot size for each modulation and coding scheme (MCS)

Having this information, the MAC level can work without knowing particular PHY details. This abstraction is also important for the BS scheduler. As an example, our implementation has a common MAC level scheduler that works correctly on top of both OFDM and OFDMa. As can be seen 
from Fig. 1, all the core components have an association to the WiMAXWirelessPhy class that abstracts particular PHY sub-classes.

\subsubsection{Effective SINR}

One of the most critical issues for a packet level simulator is how obtain a valid effective signal-to-interference noise ratio (SINR). As we will present later, once the packet level simulator has an effective SINR, it can model accurately upper PHY functionality, such as PDU errors, HARQ operations, channel reports, link adaptation etc.

Many NS-2 researchers and developers assume that the NS-2 wireless framework already addresses all the necessary PHY aspects and 802.16 PHY is just a question of changing existent parameters or choosing slightly different models [14]. Unfortunately, NS-2 is far even from capturing all the necessary 802.11 PHY aspects [17]. Its logical design corresponds to a single-carrier case, to which path loss, and optionally antenna and fading models are applied. In reality, both 802.11 [1] and 802.16 PHY rely upon the OFDM technology with multiple sub-carriers. It means that each sub-carrier can experience a different path loss, fading, interference and so on. On the one hand, in 802.11 and 802.16 OFDM PHY we can assume that all the sub-carriers have exactly the same behavior thus working with a single carrier that will represent an effective SINR. To some extent, it is a valid approach for 802.16 OFDM where a slot always maps to all the subcarriers in the OFDM symbol; the same holds for 802.11. On the other hand, sub-carriers may have quite different SINR values due to partially overlapping bandwidth (typical 802.11 case), a small guard band or interfering cells (802.16 case). Another important PHY aspect that usually NS-2 researchers do not account for is the UL sub-channelization gain. Since an SS transmission power must be distributed evenly between all the UL allocation sub-carriers, the size of the allocation has an impact on received signal strength.

Practically, it is not very complicated to introduce subcarriers in NS-2. They can be modeled quite easily over the existent Channel class without even changing the core framework. On top of that, one can add path loss, antenna pattern, shadowing/slow fading, fast fading, etc. All the related models and algorithms are well known and defined [7, 36]. The problem is that we need the effective SINR that is calculated based in individual sub-carrier SINR values. The more sub-carries we have, the more computational resources are needed. The 802.16 OFDM PHY has 256 sub-carriers; in 802.16 OFDMa, there can be up to 2048 sub-carriers, e.g., in $20 \mathrm{MHz}$ channel. Furthermore, in OFDMa PHY, sub-carriers can be either adjacent or distributed over the whole bandwidth. The problem becomes even more computationally expensive if we start to model multiple cells. It is not reasonable to turn NS-2 into another link level simulator as its power is in accurate modeling of higher layers. It is also worth mentioning that interference calculation involves heavy computations. Even system level simulators use simplifications here.

Based on these considerations, our current approach is to obtain effective SINR from trace files generated with dynamic system simulators. It allows for a good trade-off between computational complexity and accuracy. The implementation relies upon the NS-2 Propagation class, from which we derive a new class to hide trace file implementation details.

Nevertheless, we still explore possibilities to introduce a more advanced PHY model for NS-2 because the trace file approach has obvious limitations. The trace files are generated from a particular environment, particular node locations, particular traffic mixes, loads and so on. As an example, it is impossible to study the channel aware scheduling with SINR taken from the trace files.

\subsubsection{Error generation}

The error generation model is responsible for answering a question whether a received protocol data unit (PDU) is erroneous or not. To model it, we account for the way 802.16 encodes and sends data. Each data allocation, i.e., a burst, is an integer number of contiguous slots. On top of that, slots are grouped into the forward error correction (FEC) blocks, where the FEC block size depends on a particular MCS. A PDU can start and end on any byte within a data burst. All these layers are presented in Fig. 2. Thus, to model errors correctly, we have to map a received PDU to the FEC blocks it spans.

\begin{tabular}{|c|c|c|c|c|}
\hline PDU \#1 & & DU \#2 & PDU \#3 & PDUs \\
\hline FEC \#1 & FEC \#2 & FEC \#3 & FEC \#4 & FEC blocks \\
\hline & & & & Burst \\
\hline & & & & Slots \\
\hline
\end{tabular}

Figure 2. Slots, burst, FEC blocks, and PDUs

To accomplish correct PDU to FEC block mapping, we instruct the PHY layer to build a FEC block list whenever a new burst starts. Then, inside the sendUp () function, we track the number of received bytes so that whenever a new PDU arrives, we can determine the starting and ending FEC block. Once the list of FEC blocks, to which the received PDU maps, is known, it is passed to the error generation module. Each FEC block carries information on its size and SINR. The error generator applies the following formula

$$
\mathbf{E}=1-\prod_{i}\left(1-E_{i}\right)
$$

where $E_{i}$ is an individual FEC block error probability determined based on the FEC BLER curves, as Fig. 3 shows. 


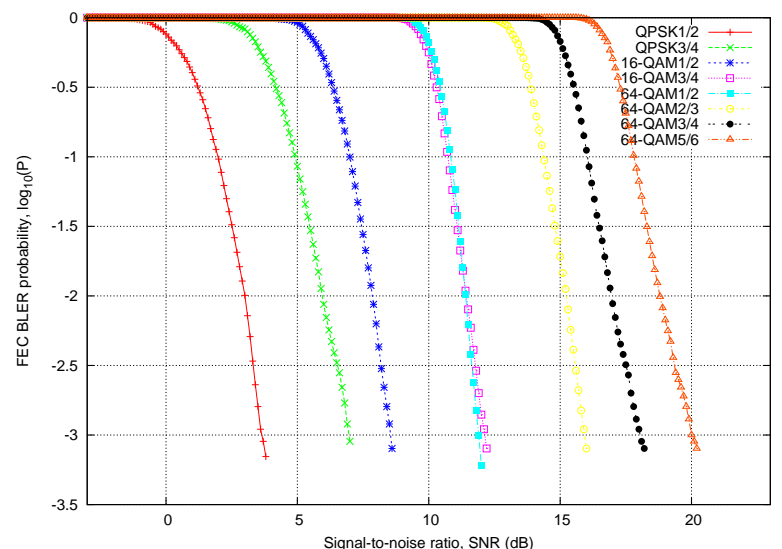

Figure 3. CTC FEC BLER curves (only the largest FEC block sizes).

It is important to note that FEC BLER curves presented in Fig. 3 are not hardcoded but rather specified at the OTcl level. It allows to switch easily between different link level simulation results and coding schemes, e.g., CC and CTC.

\subsubsection{HARQ}

The HARQ Type I, i.e., Chase Combining (CC), implementation and modeling follows the 802.16 simulation methodology [7]. Every time a new HARQ retransmission is made, the FEC block SINR from all the previous (re)transmissions is summed and submitted to the error generation module considered earlier. The HARQ Type II, Incremental Redundancy (IR), is more complicated to model with NS-2 without going into coding and decoding details. One approach is to model it on top of Type I as positive or negative gain based on retransmissions' SINR [21, 18, 22]. Another approach is to have different FEC BLER curves for the first retransmission, second one, and so on [24]. Anyway, HARQ IR mode is not so important for WiMAX networks as it is not mandated by the system profile [11].

In the case of UL HARQ transmission, the BS always knows the burst reception status. In the case of DL HARQ burst, an SS reports back the HARQ status via the HARQ ACK channel. Once the BS scheduler knows the burst reception status, it can decide whether to schedule a HARQ retransmission or continue will allocating data on next free HARQ channels.

Our HARQ implementation fully conforms to the 802.16 specification in a sense of supported and adjustable parameters. It is possible to specify the maximum number of HARQ channels (16 by default), maximum number of HARQ retransmissions ( 4 by default), HARQ buffer mode (shared by default), UL HARQ ACK delay (1 frame by default).

\subsubsection{Repetition factors}

The repetition factor is modeled similar to HARQ Type I. The received packet power is just multiplied by the repetition factor and then passed to the error generation model.

\subsubsection{Channel reports \& link adaptation}

When the BS receives data in UL, it can always estimate the channel to switch to a more suitable UL MCS in the next frame. When an SS receives data, it has to estimate the channel and report it back to the BS so that the BS link adaptation can also choose a suitable DL MCS. We support two reporting mechanisms: REP-RSP messages and CQICH channel. While the former is available in both OFDM and OFDMa PHY, it is less reliable because REP-RSP is an ordinary MAC level management message that can be dropped easily. CQICH channel, which is defined only for OFDMa PHY, provides a more robust way to report channel status.

The implementation of the REP-RSP message is quite straightforward. 6-bit CQICH messages are modeled with a special NS-2 packet type, payload of which carries the necessary information.

Once the BS scheduler link adaptation module (see Fig. 1) has information on all the SSs DL and UL SINRs, it can adjust MCS to achieve the target FEC BLER for each connection. Refer to section 4.7. for more information.

\subsection{MAC Layer}

\subsubsection{Queue system}

The general structure of the queue system is presented in Fig. 4. From the BS point of view, the air interface is a bottleneck which creates a need to buffer DL packets. Similarly, an SS needs queues to buffer UL packets. To differentiate between service flows and ensure QoS, each connection is allocated a separate queue. In addition, the BS keeps so-called UL virtual queues maintained through the bandwidth requests transmitted by SSs.

Each transport connection is equipped with several internal sub-queues where it keeps initial transmissions, retransmissions, and ARQ feedbacks. Internal priority queuing (PQ) scheduler ensures that first a connection will send ARQ feedbacks, then retransmissions, and only then normal PDUs. By the default, a sub-queue for initial transmissions relies upon a simple drop tail method. Alternatively, some Active Queue Managamenet (AQM) mechanism can be applied there. See section 4.3. for more details.

At the BS side, there is also a special DL queue where the scheduler puts generated DL-MAP, UL-MAP, DCD, and UCD messages. Besides, certain management messages, such as MOB_TRF-IND, are also placed into this queue as they are designated to all the SSs. 


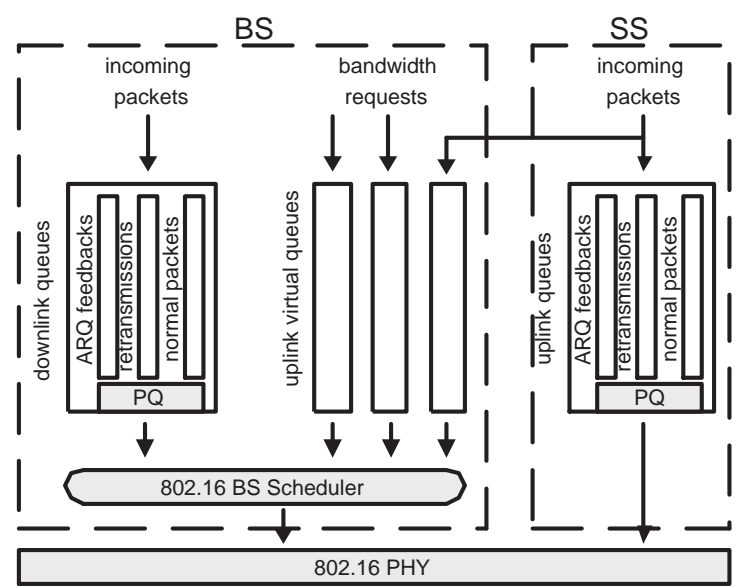

Figure 4. The queue system.

\subsubsection{Support for duplexing modes}

To support different duplexing modes, e.g., TDD, F-FDD and H-FDD, we developed a scalable design for the MAC level timers. Fig. 5 shows that there is a top level timer that elapses whenever a new frame should start. Then, a burst timer that ensures a transition from one burst to another. Whenever a new burst starts, the MAC level prepares PHY and resets certain MAC level state variables. The third level is a PDU timer that elapses whenever data transmission (or reception) ends.

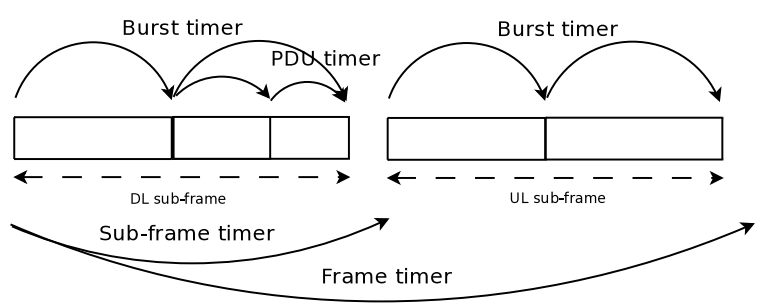

Figure 5. MAC level timers.

Fig. 5 shows a simplified timer functioning for the TDD mode where DL bursts are followed by the UL bursts. A transition from the DL sub-frame to the UL sub-frame is done through the sub-frame timer that elapses when the UL subframe starts. In the FDD mode, two burst timers function in parallel to support simultaneous data transmission and reception. Of course, the sub-frame timer is not started at all. The burst and PDU timers abstract the core MAC functionality from a particular duplexing mode.

\subsubsection{Packet header suppression}

The packet header suppression is implemented in a simple way. A script designer just specifies a constant suppression size. Whenever a packet is placed to the queue, its size is decremented to emulate suppression. Then, when a packet is passed to LL, the same constant size is added back. This functionality can be enabled on the per-connection basis thus providing different number of bytes to suppress, which may depend on the traffic type.

\subsubsection{Packing $\&$ fragmentation}

While implementing packing and fragmentation, we faced biggest problems with the NS-2 framework. There is no internal support for such a simple operation as fragmentation. This functionality must be implemented on top of NS-2 Packet class. Even worse thing is that a bad C++ design of the Packet class prevents from applying virtualization and abstraction. Packing caused even bigger problems as it implies that several NS-2 packets, or even packet fragments, are transmitted as one entity. Our solution for packing is to have a NS-2 packet with a special payload that keeps all the embedded packets or packet fragments.

\subsubsection{ARQ mechanism}

The ARQ mechanism does not contain any NS-2 specific features. Since it works on top of MAC, it is a straightforward implementation from the 802.16 specification. Once the MAC level detects missing ARQ blocks, it activates the ARQ mechanism and informs a sender to retransmit data. ARQ timers take care of situations when ARQ feedbacks are lost constantly or when retransmission attempts expire.

It makes sense to mention only one important optimization that we use in the ARQ implementation. Instead of creating and running ARQ timers for each ARQ block that a PDU has, we associate them with a whole PDU. This is an approach that also certain WiMAX products use. Of course, a special take care must be taken when later a retransmitted PDU is fragmented or packed.

The ARQ mechanism implementation supports the following features: ARQ blocks, ARQ block rearrangement, ARQ feedbacks (standalone and piggy-backed), ARQ window, ARQ timers (retry, block lifetime, Rx purge), ARQ discard.

\subsubsection{Contention resolution}

Depending on the underlying PHY, the 802.16 contention resolution works differently. Even though the top level backoff mechanism is identical to both OFDM and OFDMa PHY, the former just sends a 6-byte PDU header with the bandwidth request size. In OFDMa PHY, there are 256 144-bit pseudo-orthogonal CDMA codes. An SS sends first a CDMA contention code; once the BS detects the code, it allocates a special uplink CDMA allocation where an SS can send a bandwidth request. All these differences are abstracted through the MAC level contention resolution class.

The collision in OFDM PHY is detected and handled quite easily - if the BS detects transmission while the previous 
one has not ended yet, then all the packets are dropped. The OFDMa PHY uplink contention is trickier to model in NS2 because it is a tradeoff between accuracy and computational complexity. When several SSs transmit simultaneously CDMA codes, the BS tries to detect each of the transmitted codes. Several approaches for modeling a CDMA receiver in NS-2 are available.

1. Optimistic. A CDMA code is modeled with a special NS-2 packet type, where the packet payload carries just the CDMA code index. Since CDMA codes are pseudoorthogonal, we can assume that there is a high probability that all the transmitted codes are detected. The only case when a code collision occurs is when two or more identical codes are sent. Thus, the BS CDMA receiver just analyzes CDMA code indexes to decide whether they are detected or not.

2. Simple. This receiver is similar to the previous one with one addition - it tries to account for a inter-code interference based on the link simulation or other simulation results [20]. Even if two or more received codes have different indexes, there is a chance that a code is not detected.

3. Single-code correlator. This approach tries to model the way the CDMA codes are transmitted and detected by the CDMA receiver. The NS-2 CDMA packet payload carries 144 bits. If there are several codes sent during the same transmission opportunity, then the received bit sequences are summed to obtain a so-called interference pattern. Since each bit is BPSK modulated, there is a non-zero probability that its value changes during transmission (see Fig. 6). Thus, a receiver models it by applying the BPSK error curve to each bit. Then, the BS CDMA receiver tries to detect individual codes by applying the dot product operation and comparing the correlator output to the predefined threshold, e.g., 75\% [44]. Of course, this approach does not account for the fact that codes may arrives with different power levels. On the other hand, a properly functioning UL power control algorithm tries to ensure that received signal strength from all the SSs is approximately at the same level.

Depending the simulation accuracy and speed, an appropriate CDMA receiver can be chosen. As an example, an accurate VoIP delay analysis, where uplink ertPS connections rely upon the contention, definitely requires a complicated CDMA receiver. On the contrary, a simple CDMA receiver suffices for a basic analysis of the TCP performance over 802.16.

\subsubsection{Scanning \& handover}

In a simple single-sector simulation, there is no need for the scanning functionality as there is only one BS which an

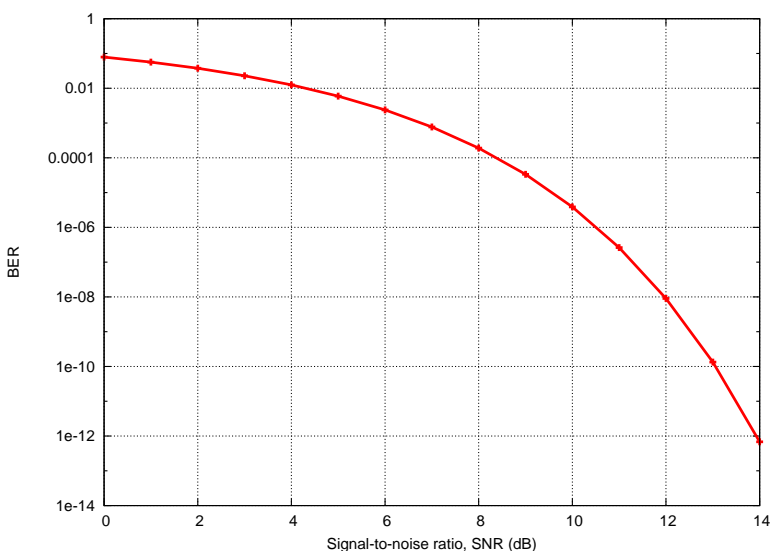

Figure 6. BPSK bit error rate.

SS will exchange data with. In this case, an SS is provided an explicit BS ID where the network entry must be performed. Otherwise, in multi-cell or relay simulations, an SS first listens for the DL channel and then enters the network via the BS that has the strongest DL signal. The scanning time is a configurable parameter; usually, $50-100 \mathrm{~ms}$ is enough to filter out the fast fading component and obtain an average DL channel performance.

Handover is a part of the basic MAC functionality and includes a support for all the necessary management messages to initiate and control the handover process. Our implementation supports two basic handover mathods: manual and an automatic one. In the former case, at a particular moment of time an SS is instructed to handover to the specified BS. In a case of the automatic handover, an SS measures periodically DL preambles from neighboring BSs and initiates a handover process once the strongest neighboring BS signal strength exceeds the hysteresis margin and stays for more than time-totrigger seconds. If for some reason the handover fails, an SS falls back to the initial network entry mode and starts to perform scanning. It allows us to simulate large mobility scenarios containing multiple BSs and random movement of SSs.

\subsection{LL layer}

The LL layer has the least amount of extensions when compared to MAC or PHY. Following the general principles, there is a derived class that changes behavior of the virtual recv() function. Firstly, we disable Address Resolution Protocol (ARP) because it does not have much sense in the 802.16 PMP mode. Secondly, the LL layer has to stamp a packet with a correct CID so that the queue system can place it into a correct queue. Practically, the NS-2 LL layer performs functions of the 802.16 convergence sublayer. This functionality is identical for an SS and BS as both of them have to classify incoming packets. Current implementation classifies packets based on the following parameters: a) source address, b) des- 
tination address, and c) flow ID. The reason we also account for flow ID is that we have to differentiate somehow between incoming packets that belong to different applications from the same node. If they belong to different application types, e.g., VoIP and BE, then they should be placed into different connection queues to obtain an appropriate treatment. On the other hand, by not specifying the flow ID we will put all the incoming packets into one connection queue which is also a valid case. A script designer decides which option to use.

\subsection{QoS and scheduling}

The internal QoS architecture of our 802.16 module does not differ significantly from QoS architectures presented in other papers and 802.16 implementations $[19,16]$. Thus, for the sake of brevity, we focus only on distinctive features we adopted in our module.

\subsubsection{BS scheduler}

We omit the description of the BS scheduler details as more information is given in section 4.1.. However, we mention briefly that to study different schedulers in 802.16, we introduced a common interface between the BS MAC and the BS scheduler, as Table 2 presents.

Table 2. BS scheduler interface.

\begin{tabular}{|l|l|}
\hline Input & Output \\
\hline DL queue sizes & DL burst list \\
UL queue sizes & UL burst list \\
HARQ ACKs & DL-MAP, UL-MAP, DCD, UCD \\
\hline
\end{tabular}

The input parameters are the status of DL and UL virtual queues (see Fig. 4) that are maintained and managed by the MAC layer. In addition, we also pass HARQ ACKs because they arrive to BS via the MAC level. The result of the scheduling decision is two lists with DL and UL bursts for the BS MAC. The scheduler also constructs the DL-MAP, UL-MAP, $\mathrm{DCD}$, and UCD messages that the BS MAC transmits later to all the SSs.

\subsubsection{SS uplink scheduler}

The SS uplink scheduler is as important as the BS scheduler. The reason is that once the BS makes an UL allocation, it is per a whole SS, not per an individual connection. Then, it is the SS responsibility to partition this UL allocation between multiple transport connections, if any. Such a solution aims at reducing the signaling overhead and allowing an SS to gain a better control on the allocation size, which can start and end at any byte within the burst. Fig. 7 shows an example of how an UL burst can be shared between several connection types.

The default uplink scheduler in our implementation is PQ. Its simple yet efficient design allows for supporting triple-

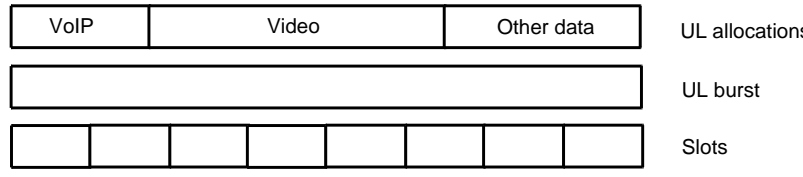

Figure 7. UL burst partitioning.

play services at mobile terminals. In other words, first the uplink scheduler allocates space for the management data, if any, then UGS, then ertPS (e.g., VoIP), then rtPS (e.g., video), and then nrtPS (e.g., Web) with BE.

Of course, the PQ scheduler cannot address more complicated and general cases when, as an example, there are several nrtPS connections with diverse QoS requirements, such as the 802.16 CPE that serves a small local network. To experiment with different uplink schedulers, we developed a common interface that allows to abstract from a particular allocation algorithm (see Table 3). Input parameters are time, UL allocation size, and the queue sizes. Output information is a list with UL allocation sizes that tell how many bytes each connection may occupy. Then, it is the MAC level responsibility to enforce this decision.

Table 3. Uplink scheduler interface.

\begin{tabular}{|l|l|}
\hline Input & Output \\
\hline Time & UL allocation list \\
UL allocation size & \\
UL queue sizes & \\
\hline
\end{tabular}

\subsection{16j multi-hop extensions}

Multi-hop extensions [9] were added recently to the baseline 802.16e specification and allow for deployment of various relay types with different operational modes. In this paper we refrain from describing all the $802.16 \mathrm{j}$ technical details, a good overview of the key $802.16 \mathrm{j}$ features can be found in [34].

The key concept behind implementing a scalable support for the multi-hop functionality is a zone. Being part of the basic 802.16 specification, it allows for partitioning the DL/UL sub-frame into smaller logical parts by using the time division concept. Originally, this approach was introduced to support different permutation type within the same sub-frame, e.g., PUSC, FUSC, AMC. In the 802.16j extensions, a zone also specifies whether it is used for communication with SSs or with a relay stations (RS).

WINSE supports 2-hop non-transparent time-division transmit relays as a main solution for the coverage extension problem [4]. Transparent relays, which are used merely for the throughput enhancement within a cell coverage area, are left for the next stage. Even though the specification allows for more than 2 hops, such a possibility is not supported due to the resulting system complexity [10]; the same approach 
is also taken in other technologies, such as 3GPP. As a result, there is no need to support more than one DL/UL relay zone in the 802.16 frame. Further WINSE development may include a support for an unlimited number of hops.

\subsubsection{PHY, MAC, queue system}

Since the 802.16j specification does not define any new PHY features, we rely upon the same PHY concepts and principles described earlier. The only enhancement we introduced is a set of propagation models defined by the $802.16 \mathrm{j}$ simulation methodology [5]. All the other PHY mechanisms, such as channel measurements, reporting, link adaptation, are exactly the same as for SS and BS.

Obviously, the MAC level has the largest amount of extensions. Due to the scalable archicture, the RS MAC level is a combination of the BS and SS functionality, as Fig. 1 illustrates. Indeed, RS behaves like a full-featured BS while scheduling resources for its access link, sending and receiving data there. At the same time, the RS node behaves like an SS when it follows the scheduling decision made by BS on the relay link.

The queue system is identical to the MAC level queue with one small extension - it is capable of buffering both the DL and UL PDUs, because depending on the channel conditions RS may have to buffer both DL and UL traffic.

\subsubsection{Scheduling}

Scheduling was one of the most challenging tasks while implementing a support for $802.16 \mathrm{j}$. In addition to providing resources for SSs, as in a basic 802.16 network, BS has to allocate slots on a relay link to exchange data with RSs. To accomplish this task, we introduced a concept of a scheduling group, where each group is an independent set of resources managed by BS. Then, a DL and UL relay zones form a separate scheduling group, whereas DL and UL resources for an access link belong to a different scheduling group.

In addition to the DL and UL scheduler that assigns resources on the RS access link, the RS node has to run a socalled UL allocation scheduler. Similarly to an SS, the BS grants an UL allocation to a whole RS by using its basic management CID; then, it is RS responsibility to share it between all the associated stations. In 3.6.2., we presented an architecture of the SS UL scheduler and described a simple resource allocation mechanism based on PQ. Even though it suffices for an SS, it is not flexible for the RS node. The biggest obstacle is that due to the PQ nature, one BE TCP connection can monopolize easily all the resources. Instead, we implemented a PQ-RR scheduler that prioritizes management, UGS and ertPS connections by using strict priorities. Connections that belong to remaining classes, i.e., rtPS, nrtPS, BE, are shared with a round-robin scheduler. Such a scheme combines simplicity and efficiency assuming that the BS UL scheduler ac- counts for the QoS requirements of the RS connections and allocates sufficiently large UL grants.

\subsection{Routing}

While working in the PMP mode, packets are always exchanged between SSs and the BS belonging to the same cell - there is no way an SS can communicate to the other one bypassing the BS. As a result, there is no need in the ad-hoc routing protocol because all the forwarding information becomes available during a connection set up. To reflect this fact, we rely upon the NOAH module that just disables adhoc routing.

\subsection{Tracing}

Following one of the core principles, we rely upon the trace format provided by NS-2 framework. We do not change the trace file format as it will break existent scripts or start to conflict with other extensions. However, there is 802.16 specific information that one would like to see in the trace file for further analysis and testing.

\subsection{6 specific MAC fields}

The NS-2 wireless trace file format defines four $-\mathrm{Mxxx}$ fields, content of which is specific to a particular MAC level. We redefine them to include 802.16 specific information, as Table 4 presents. The - Mt field always have the same value, 802.16, which allows to differentiate from other MAC types. The - Mc field keeps the CID value. It helps to differentiate between several connections belonging to same SS or just track down a particular connection. The $-\mathrm{Mm}$ field specifies the management message type if a transmitted PDU belongs to the management connection. Having the management message type in the trace file, one can gather plenty of important information. As an example, network entry delay (time between the first RNG-REQ and the last REG-RSP messages), connection setup delay (time between the first DSAREQ and the last DSA-ACK messages), handover delay (time between the first MOB_MSHO-REQ and the last REG-RSP messages), and so on. The $-\mathrm{Mb}$ field specifies the bandwidth request size of a standalone or piggy-backed bandwidth request.

Table 4. New trace fields.

\begin{tabular}{|l|l|}
\hline Field & Description \\
\hline$-\mathrm{Mt}$ & 802.16 \\
$-\mathrm{Mc}$ & $\mathrm{CID}$ \\
$-\mathrm{Mm}$ & management message type \\
$-\mathrm{Mb}$ & bandwidth request size \\
\hline
\end{tabular}




\subsubsection{Burst entry}

Even though the NS-2 trace file contains information on all the transmitted PDUs, sometimes it is not enough to analyze the resulting 802.16 behavior. Firstly, the NS-2 format does not provide enough $-\mathrm{Mxxx}$ fields to display other useful information, such as MCS or the HARQ (re-)transmission status. Secondly, 802.16 data transmission occurs in a form of a data burst that comprises one or more PDUs. Thus, we also add a support for a so-called "burst" entry.

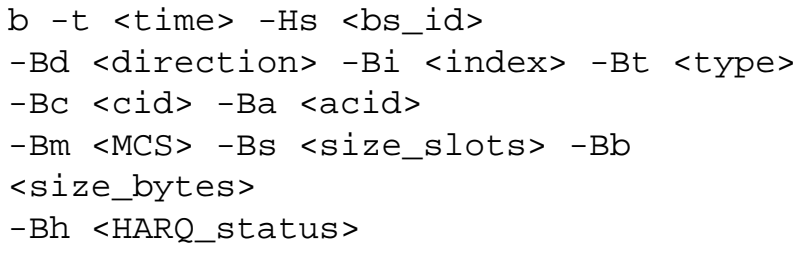

Figure 8. Format of the burst entry.

Fig. 8 shows the format of the "burst" entry that appears in the trace file whenever a new burst starts. The entry includes information on a burst direction, its index, type, e.g., data burst or a contention region, CID and ACID values, burst MCS, size in slots and bytes, as well as the HARQ status for the HARQ-enabled data burst. It allows to gather statistics, such as MCS distribution, burst size distribution, distribution of HARQ retransmissions etc.

\subsubsection{Contention entry}

We add a special "c" entry to the trace file. It specifies when an SS takes part in the uplink contention resolution. The reason we need this entry is that when an SS starts the uplink contention, it may defer for a number of frames due to the backoff start value. As a result, the first bandwidth request is sent later when the actual uplink contention has begun. Thus, the "c" entry helps to measure accurately the medium access delay. Since an SS performs the uplink contention on behalf of all the connections it has, the format of this entry is very simple, as Fig. 9 presents. There is time when a contention starts, SS node ID that originates the contention, and the BS node ID to which the contention request is sent.

c -t <time $>-\mathrm{Hs}_{\mathrm{s}}<\mathrm{s}$ _ id $>-\mathrm{Hd}<\mathrm{bs}$ _id $>$

Figure 9. Format of the contention entry.

\subsubsection{Drop reason}

There are many reasons why a packet can be dropped inside the MAC layer. We rely upon the existent NS-2 drop reasons to put appropriate information to the trace file.

\subsection{Access network}

Along with the 802.16 radio interfaces, the performance of the WiMAX network is affected by the wired part. In fact,
Table 5. Drop reasons.

\begin{tabular}{|l|l|}
\hline Drop reason & Description \\
\hline IFQ & The queue is full \\
ERR & Packet error \\
COL & Uplink contention collision (OFDM only) \\
RET & Contention attempts exceeded \\
NRTE & No classification rule \\
\hline
\end{tabular}

wired network components, such as access service network gateway (ASN-GW) and connectivity service network (CSN) node (see Fig. 10), may play a crucial role during network entry, connection setup, and handover processes because the BS contacts them at various stages [8]. Failing to model the access network, one can obtain too optimistic results. Apart from time needed to send a signaling message and wait for a response, there are also delays that may come from the user data preempting management traffic inside the access network.

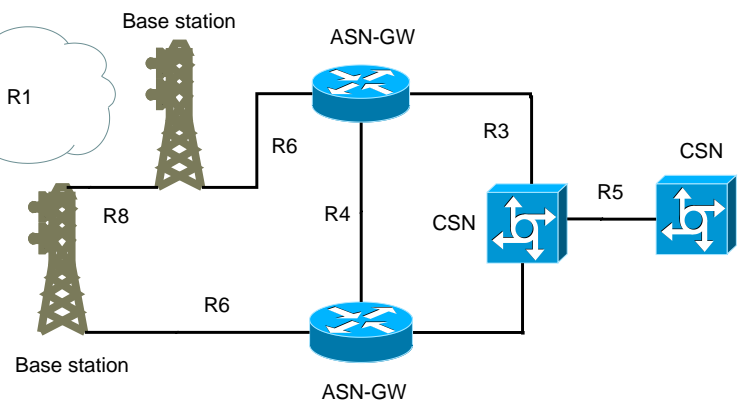

Figure 10. WiMAX access network components and interfaces.

Access network modeling combined with 802.16 PHY and MAC is a perfect task for a simulator, such as NS-2, where wired networking has been present for a long time. Besides, NS-2 provides a good framework to develop new protocols, in particular signaling ones.

We already developed an extension to the WINSE module that aims at adding a support for the 802.16 access network entities to the NS-2 simulator. Our primary goal is to support R4, R6, and R8 interfaces to obtain more accurate results for network entry and handover procedures. The description of the module with simulation results is given [32]

\section{RESEARCH DONE WITH WINSE}

In this section we give a brief overview of past and ongoing research topics where the WINSE is used.

\subsection{Scheduling \& resource allocation}

Scheduling and resource allocation was the first research topic studied with the WINSE extension [42, 41]. It was 
shown that the classical fair resource allocation is applicable but is too complicated for the 802.16 networks, where the basic allocation unit is a slot of a fixed size and duration. As a result, a simpler yet efficient algorithm is possible that is based conceptually on the round-robin approach. At the same time, there were proposed extensions that allowed for accounting for the 802.16 service class, QoS parameters, the UL bandwidth request or DL queue size. The simulation results showed that the proposed scheme ensures all the QoS requirements, protects service flows, and ensures fair resource allocation across the BE connections. All our subsequent research papers relied upon the proposed scheduler that was adopted easily for the OFDMa PHY. Its simple but scalable nature also allowed to introduce an extension for ARQ [40] and a support for the HARQ scheduler.

Due to a highly modular internal architecture, other schedulers, such as proportional fair, were studied with the WINSE module [27].

\subsection{Uplink contention performance}

Even though the 802.16 system is based on the demand assigned multiple access (DAMA) concept, there is still an uplink contention created by connections that are either not polled regularly, e.g., nrtPS, or are not polled at all, such as BE. The ertPS class can also take part in the uplink contention if so allowed by the BS. In [39], we studied the 802.16 uplink contention resolution and proposed a scheme on how to adapt dynamically backoff start/end parameters, and the number of the contention transmission opportunities. Furthermore, we showed in [38] that by adjusting dynamically the uplink contention parameters one can achieve a good tradeoff between resource utilization and delay requirements of the VoIP connections.

Another 802.16 feature to achieve the tradeoff between delay guarantees and resource utilization is multicast polling. In [12], a study was done to research the applicability of it with VoIP connections. It was shown that the multicast polling can be used to provide a maximum delay guarantee and even several separate delay limits for separate connection types. As a continuation of the uplink contention and VoIP topics, we also compared different resumption mechanisms in OFDMa PHY [25].

Our recent paper studied the OFDMa PHY CDMA contention code performance and how they can be optimized for future broadband wireless systems [20].

\subsection{AQM}

From the SS and BS point of view, the 802.16 network is a bottleneck because it is highly anticipated that a wired connection behind BS (or SS working as a gateway) will be always higher than the maximum achievable throughput on the air interface. Thus, the queue sizes tend to grow especially when spectrum efficiency declines. In [26], we studied AQM mechanisms that it is possible to apply to 802.16 to reduce queuing delays. The results showed that being applied to the BS DL queues, the AQM mechanism is capable of reducing TCP delays.

\subsection{Optimal PDU Size}

While transmitting data, incoming packets, i.e., SDUs, are fragmented or packed into PDUs, size of which is not governed by the specification. On the one hand, the propability of errornous MAC PDU increases when the PDU size grows. On the other hand, a small PDU has a larger overhead. In [31], we studied the optimal MAC level PDU size in different channel conditions. We showed that it is possible to find the optimal PDU size if we know the channel conditions. Because 802.16 system has an advanced link adaptation mechanism, the error probability is known and the optimal PDU size can be selected. It was also shown that if the error probability is unknown, then it is better to rely on smaller PDU sizes of around 100-120 bytes.

\subsection{ARQ}

The ARQ retransmission mechanism is available in all the major PHYs of the 802.16 system and plays a key role in improving the system performance, especially the application level throughput. In [45], we studied the properties of the 802.16 ARQ mechanism and proposed solutions on how to choose an ARQ feedback type, prioritize feedbacks, retransmissions, and normal PDUs. We showed the importance of the ARQ block rearrangement and a correctly set up ARQ transmission window. In [46], we analyzed the impact of the ARQ feedback intensity in the case when ARQ works standalone and on top of HARQ.

In [30], we studied performance of the ARQ mechanism with the real-time VoIP traffic. We presented that even though HARQ is considered to be a better candidate for VoIP, it is possible to tune the ARQ algorithm so that all the VoIP performance requirements are met.

\subsection{ARQ \& HARQ performance}

Along with ARQ, the 802.16 OFDMa PHY provides a possibility to run the HARQ retransmission mechanism. In [43], we made a preliminary comparison of these two mechanisms and also studied ARQ on top of HARQ. Even though, as expected, HARQ outperforms ARQ in most cases due to a retransmission gain, there are cases when ARQ provides a better performance due to less signaling information.

\subsection{Link Adaptation Thresholds}

In [35], we have analyzed the link adaptation model and MCS transition thresholds for the IEEE 802.16 base station. 
We have shown that the optimal transition threshold for the ARQ connections is between $10^{-2}$ and $10^{-2.5}$, while for the HARQ enabled connections it is from $10^{-1.5}$ to $10^{-2}$. It fully conforms to the theoretical expectations that HARQ should outperform ARQ due to the retransmission gain. An interesting outcome of the paper is that the optimal thresholds depend on the number of data bursts per a frame. It requires a coordinated functioning between the BS link adaptation model and the scheduler.

\section{ONGOING RESEARCH TOPICS}

In this section we provide some simulation results of our ongoing research topics, which are a comparison of different duplexing modes in 802.16 and $802.16 \mathrm{j}$ relay performance.

\subsection{TDD vs. F-FDD vs. H-FDD}

In this sub-section we present the simulation results for different duplexing modes that our simulator supports. We consider the time-division and frequency-division duplexing, where the frequency-division is further classified into full mode and half-duplex modes. In the half-duplex mode, the 802.16 DL/UL sub-frames are partitioned into two groups to support stations that cannot transmit and receive simultaneously. In addition, two functional modes are analyzed for TDD: when UL-MAP points to the current frame and to the next one.

Table 6. TDD/F-FDD/H-FDD PHY parameters.

\begin{tabular}{|c|c|c|c|}
\hline Parameter & TDD & F-FDD & H-FDD \\
\hline PHY & \multicolumn{3}{|c|}{ OFDMa } \\
\hline Cyclic prefix length & \multicolumn{3}{|c|}{$1 / 8$} \\
\hline Frames duration & \multicolumn{3}{|c|}{$5 \mathrm{~ms}$} \\
\hline Long preamble & \multicolumn{3}{|c|}{1 symbol } \\
\hline Bandwidth & $10 \mathrm{MHz}$ & \multicolumn{2}{|c|}{$5+5 \mathrm{MHz}$} \\
\hline FFT & 1024 & \multicolumn{2}{|c|}{512} \\
\hline TTG+RTG & $296+168$ PS & \multicolumn{2}{|c|}{$0+168$ PS } \\
\hline OFDM symbols & 47 & \multicolumn{2}{|c|}{45} \\
\hline \multirow{2}{*}{$\begin{array}{l}\text { DL/UL symbols } \\
\text { DL/UL permutation }\end{array}$} & $22 / 24$ & $44 / 42$ & $12+32 / 12+30$ \\
\hline & \multicolumn{3}{|c|}{ DL PUSC / UL PUSC } \\
\hline DL slots & 330 & 330 & $90+240$ \\
\hline UL slots & 280 & 238 & $68+170$ \\
\hline
\end{tabular}

Table 6 presents the PHY parameters for this simulation. It is worth mentioning that $10 \mathrm{MHz}$ frequency band used in TDD is partitioned into two $5 \mathrm{MHz}$ bands used by $\mathrm{DL}$ and UL carriers in FDD. Further, depending on the FDD mode, either all the OFDM symbols are used for transmission or they are paritioned into two groups in the H-FDD mode. The TDD DL/UL ratio is chosen in such a way that the number of slots is DL and UL sub-frames is comparable to FDD. Table 7 presents the common MAC level parameters that do not depend on a particular duplexing mode.

Fig. 11 shows the DL and UL spectral efficiency for the considered duplexing and functional modes. It can be seen that results do not vary a lot. It is explained by the fact that the BS Tx power is the same and the operational parameters,
Table 7. Common MAC parameters.

\begin{tabular}{|l|l|}
\hline Parameter & Value \\
\hline DL/UL channel measurements & preamble / data burst \\
Channel report type / interval & CQICH / 20ms \\
Channel measurements filter & EWMA, $\alpha=0.25$ \\
\hline MAP MCS & QPSK1/2 Rep6 - QPSK1/2 \\
Compressed MAP/sub-MAPS & ON/ON \\
Maximum sub-MAPS & 3 \\
\hline Ranging transm. opport. & 2 \\
Ranging backoff start/end & $1 / 15$ \\
Request transm. opport. & 8 \\
Request backoff start/end & $3 / 15$ \\
CDMA codes & 256 \\
ranging+periodic ranging & 64 \\
bandwidth request & 192 \\
handover & - \\
\hline Fragmentation/packing & ON \\
CRC & ON \\
\hline ARQ feedback & standalone \\
ARQ feedback types & all \\
ARQ feedback interval & $20 \mathrm{~ms}$ \\
ARQ block size & $64 \mathrm{~B}$ \\
ARQ window & 1024 \\
ARQ block rearrangement & ON \\
ARQ deliver in order & ON \\
ARQ timers & $100 \mathrm{~ms}$ \\
retry & $500 \mathrm{~ms}$ \\
block lifetime & $500 \mathrm{~ms}$ \\
Rx purge & \\
\hline
\end{tabular}

i.e., number of OFDM symbols, are chosen in such a way that the amount of resources in DL is roughly the same for both TDD and FDD. Nevertheless, it is noticeable that TDD with the UL-MAP pointing to the next frame provides slightly better results. Good DL performance of H-FDD in case of 2 SSs is explained by fixed H-FDD group ratio that makes it quite difficult to achieve a good throughput fairness in case of a few stations. In UL, TDD outperforms FDD due to fact that there are less slots available for data transmission (see Table 6). This is explained by the 802.16 UL PUSC design where a slot comprises 3 OFDM symbols. As a result, a few symbols always remain unused.

Fig. 12 presents the DL and UL throughput CDF for a particular case of 24 stations. In case of DL, the throughput $\mathrm{CDF}$ is almost the same for all the duplexing modes. As already considered earlier, FDD provides worse results in case of the UL data transmission due to less amount of available resources. In addition, H-FDD throughput CDF spans a larger range because the BS scheduler is less flexible in assigning resources for SSs that are associated with different H-FDD groups of the fixed size.

It must be mentioned that theoretically the FDD duplexing mode should be as good as TDD in the UL direction or even better. The reason for it is the better UL sub-channelization gain for FDD. Due to a longer UL sub-frame size, an SS can transmit more data in one sub-channel when compared to TDD. The reason we obtain worse results for FDD in UL is absence of a proper UL power control module that can benefit from the UL sub-channelization in FDD. 


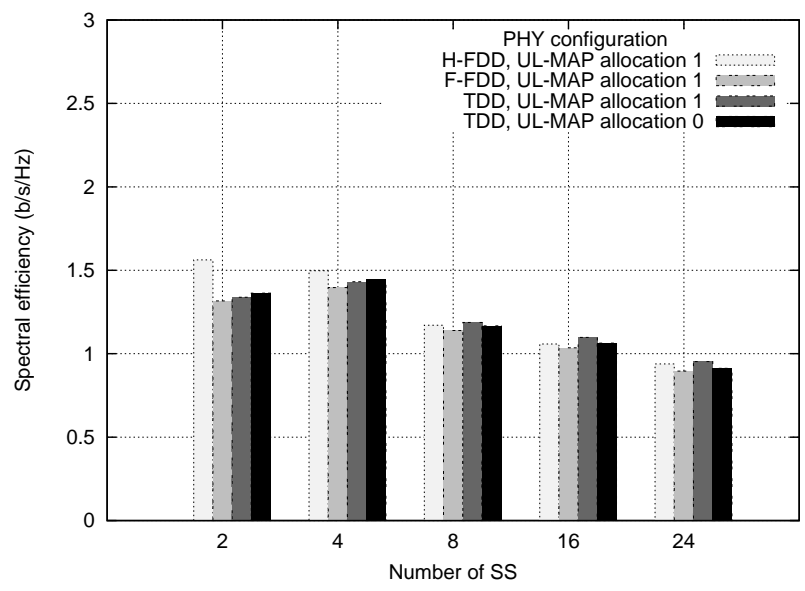

(a) $\mathrm{DL}$

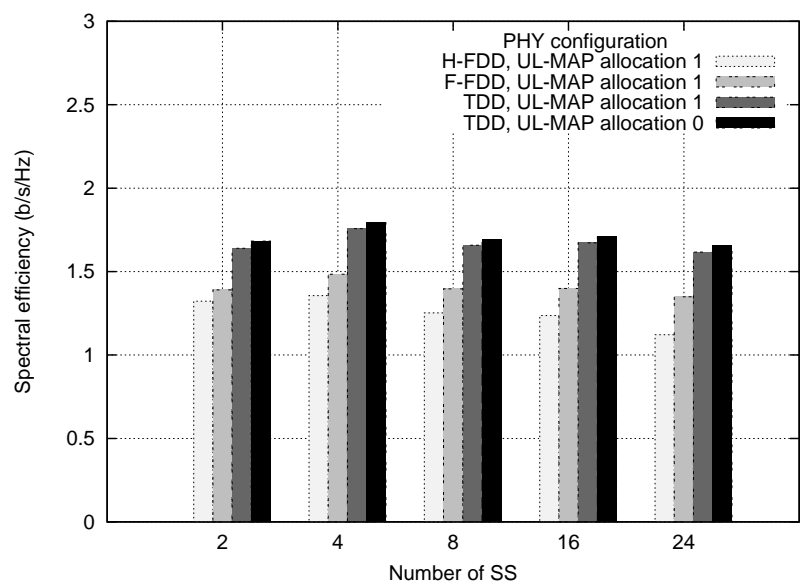

(b) UL

Figure 11. Spectral efficiency.

\subsection{16j non-transparent relays}

Fig. 13 shows the relay simulation scenario. It is assumed that there is a single BS controlling its sector. To serve an area denoted by a dashed line, an operator may deploy additional BSs to cover two more sectors denoted by dotted lines. However, a more cost efficient solution might be to deploy a few relay nodes [28], as shown in the figure. For the sake of brevity, we compare a case with a single BS and a case with the BS and RS nodes.

Table 8 presents the key parameters used in the simulation. We consider the DL TCP transmission over the BE connections as it is a good way to analyze the resulting system throughput and the spectral efficiency. It is worth mentioning that to study the relay performance, we consider different fixed DL relay zones size, i.e., 2,4, and 6 OFDM symbols. Also, unlike the BS, RS uses an omnidirectional antenna, has a lower Tx power of $5 \mathrm{~W}$ and a smaller antenna height. We assume the sub-urban macro-cell scenario and thus choose

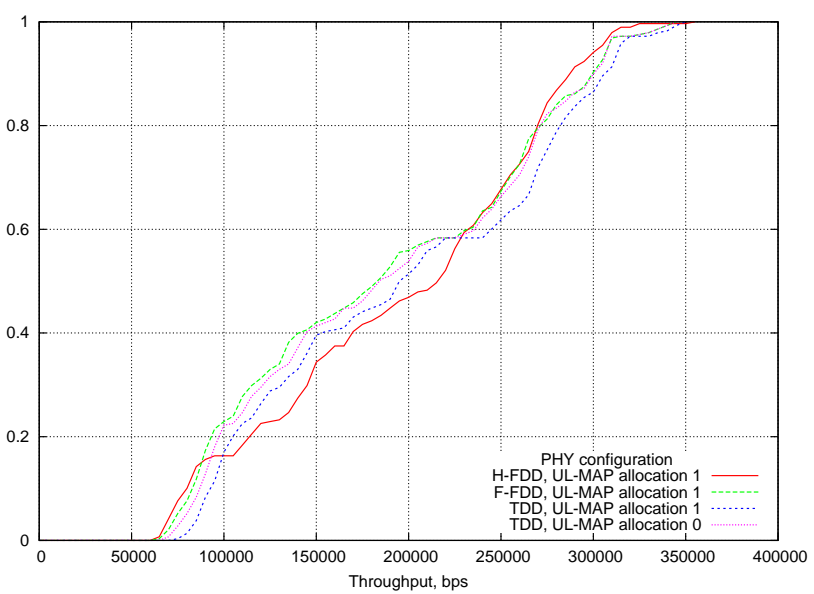

(a) DL

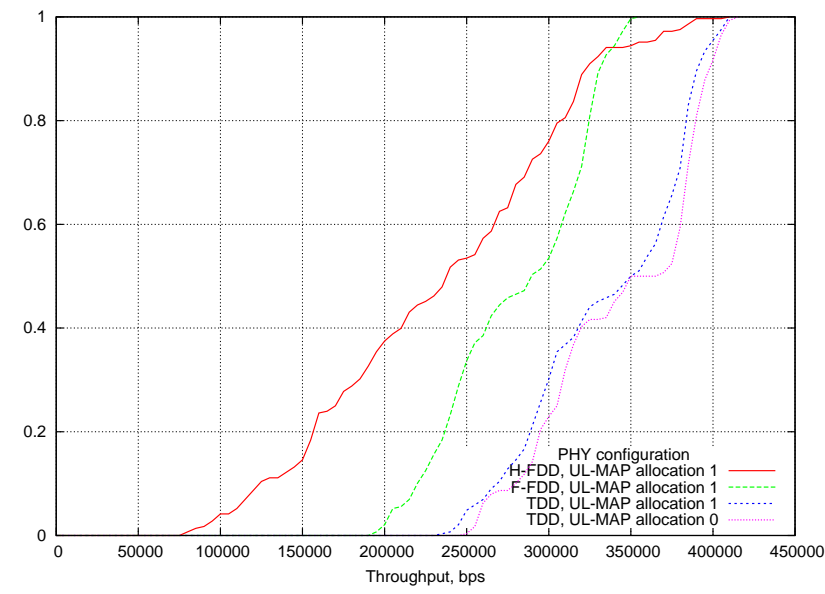

(b) UL

Figure 12. Throughput cumulative distribution.

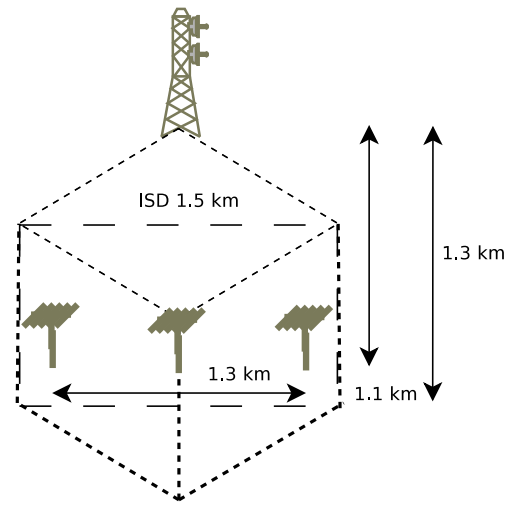

Figure 13. Simulation scenario.

802.16m SMa propagation model to for an access link and 802.16j TypeD model for the relay link. The retransmission mechanism is ARQ working in the end-to-end mode. In other words, RS does not take part in the ARQ signaling and just 
forwards received data. The interference modeling accounts for the fact that BS and the non-transparent RS transmit simultaneously thus impacting each other.

Table 8. 802.16 network parameters.

\begin{tabular}{|l|l|}
\hline Parameter & Value \\
\hline PHY & OFDMa \\
Bandwidth & $10 \mathrm{MHz}$ \\
Duplexing mode & TDD \\
Frame duration & $5 \mathrm{~ms}$ \\
CP length & $1 / 8$ symbol \\
OFDM symbols & 47 \\
DL/UL symbols & $30 / 15$ \\
DL/UL relay zone size & $2,4,6$ / 3 symbols \\
DL/UL subcarrier alloc. & DL PUSC/UL PUSC \\
Channel report type / interval & CQICH / 20ms \\
Channel measurements DL/UL & preamble / data burst \\
Channel measurements filter & EWMA, $\alpha=0.25$ \\
\hline Compressed MAPs & ON \\
sub-MAPs & ON, max. 3 \\
\hline BS / RS / SS Tx power & $10 / 5 / 0.25 \mathrm{~W}$ \\
BS / RS / SS antenna & $3 \mathrm{GPP} /$ omni / omni \\
BS / RS / SS antenna height & $32 / 12 / 1.5 \mathrm{~m}$ \\
A-link / R-link path loss & $.16 \mathrm{~m} \mathrm{SMa/.16j} \mathrm{TypeD}$ \\
\hline Ranging transm. opport. & 2 \\
Ranging backoff start/end & $1 / 15$ \\
Request transm. opport. & 8 \\
Request backoff start/end & $3 / 15$ \\
CDMA codes & 256 \\
ranging+periodic ranging & 64 \\
bandwidth request & 192 \\
handover & - \\
\hline PDU size & $140 \mathrm{~B}$ \\
Fragmentation & ON \\
ARQ feedback & standalone \\
ARQ feedback types & all \\
ARQ feedback intensity & $20 \mathrm{~ms}$ \\
ARQ block size & $64 \mathrm{~B}$ \\
ARQ window & $1024 \mathrm{~B}$ \\
ARQ discard & ON \\
ARQ block rearrangement & ON \\
ARQ deliver in order & ON \\
ARQ timers & $100 \mathrm{~ms}$ \\
retry & $500 \mathrm{~ms}$ \\
block lifetime/Rx purge & \\
\hline
\end{tabular}

To gather statistically reliable results, we run 20 different simulations, where each simulation run contains 30 nodes placed in random locations. Each simulation run lasts for 10 seconds. Fig. 14 presents the simulation area with SS locations and their associations to BS or RS node. Note that Fig. 14 accumulates results from all the simulation runs, i.e., 600 different locations. It must be mentioned that we do not put SSs close the BS as they experience very good SNR there and thus are not so interesting for this relay study.

Fig. 15 presents the DL spectral efficiency for a case when there is only the BS (the leftmost bar) and three cases with relays and different DL relay zone sizes (2,4, and 6 symbols). As can be seen, relays improve the spectral efficiency: the more resources a relay link has, the better an average spectral efficiency becomes because SSs, which are close to RSs, can benefit from a good link between BS and RS.

To provide a better insight on the relay performance, we also present the DL throughput CDF in Fig. 16. It shows that relays improve performance, but there is a certain number of SSs that has worse throughput. The reason is that these

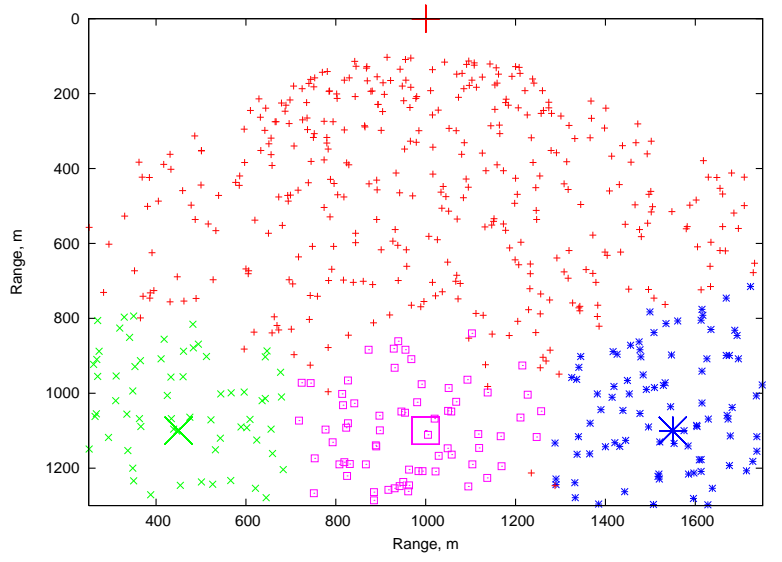

Figure 14. Simulation area with SS locations.

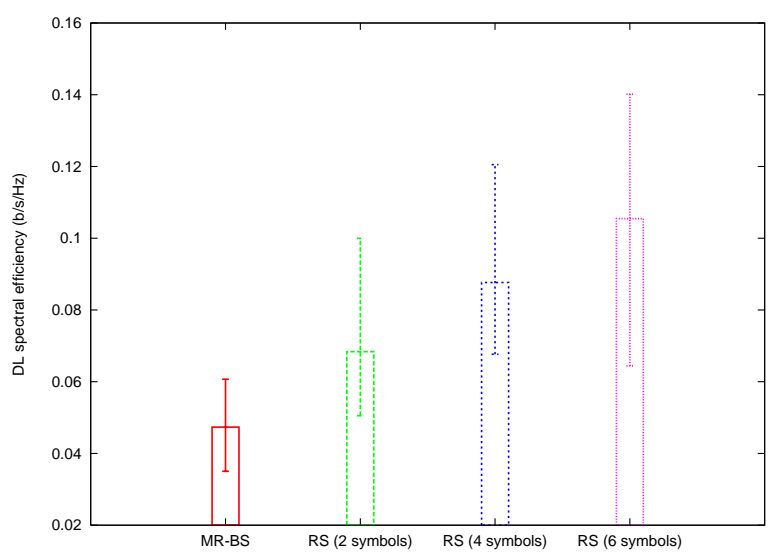

Figure 15. DL spectral efficiency.

SSs reside in very bad channel conditions, e.g., cell edge or BS/RS coverage areas intersection. Their performance gets worse due to the increased interference level when we deploy non-transparent relays.

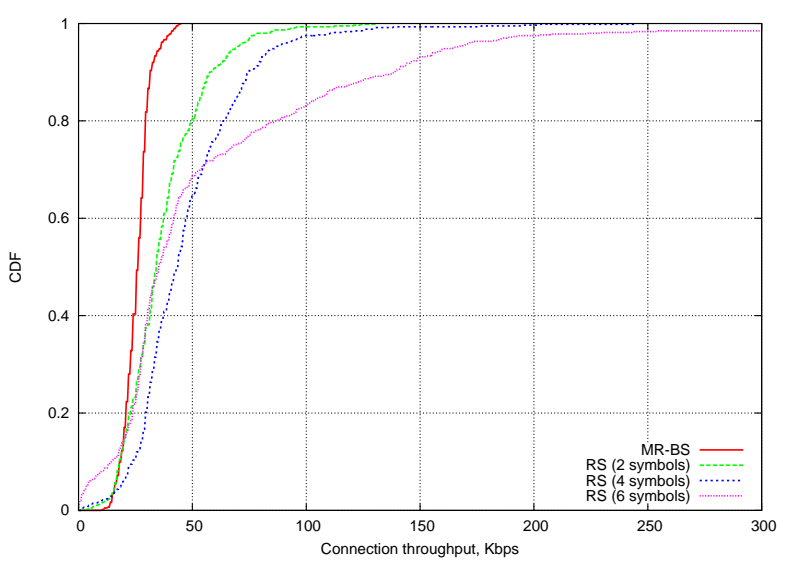

Figure 16. DL throughput cumulative distribution. 
Fig. 17 presents an analysis of throughput fairness. ${ }^{1}$ Of course, if there is only the BS node then it can ensure quite high throughput fairness because the BS scheduler has a complete control over resource allocation on the access link. Once we deploy relays, everything the BS scheduler can do is to control resources for the relay link, but not the way the RS node will allocate resources between connections on its access link. It can be seen that as we increase the DL relay zone size, the throughput fairness gets worse and worse. The explanation is that a few SSs associated with one of the RS nodes can transmit more data. At the same time, SSs associated with BS have less resources on the access link due to the increased DL relay zone size.

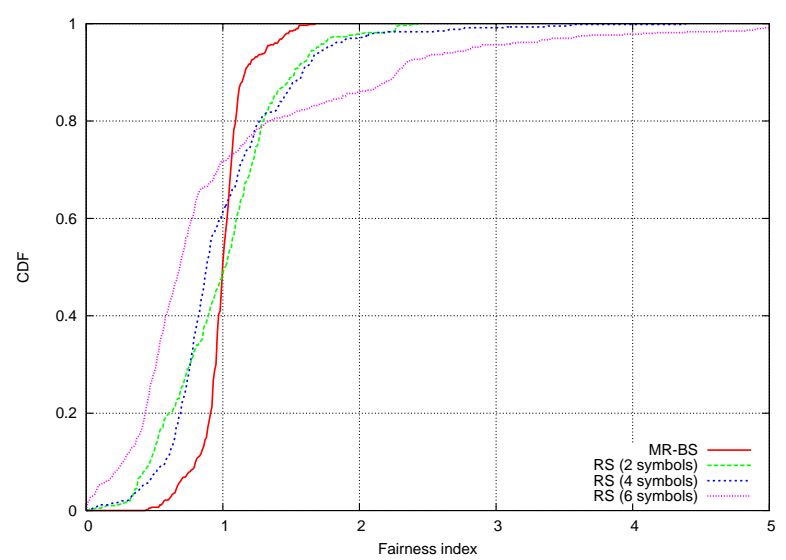

Figure 17. DL throughput fairness.

As a small summary, relays indeed improve the overall system performance at the expense of the overall throughput fairness that is quite difficult to achieve in the multi-hop environment. Furthermore, non-transparent relays increase the interference level that affect negatively performance of SSs that reside in very bad channel conditions. The relay zone sizes must be set up carefully as they control a trade-off between the system performance and fairness. For the simulation simulation scenario presented above, the DL relay zone size of 4 symbols is good compromise between the increased spectral efficiency and decreased throughput fairness.

\section{CONCLUSIONS}

The paper has presented the 802.16 module for the NS2 simulator, called WINSE. We introduced a support for the upper PHY level, MAC, QoS and scheduling, as well as the basic support for the access service network. Our implementation has proved that NS-2 can be used to model complicated wireless broadband technologies, such as IEEE 802.16. We used the WINSE module in many research papers where we

\footnotetext{
${ }^{1}$ We use the fairness index defined in [7]. An absolute fair throughput allocation yields a vertical line with the x coordinate of 1 .
}

studied the MAC and QoS aspects of the IEEE 802.16 system. A number of technical contributions were also submitted.

Based on our experience, it is possible to state that a truly powerful and scalable NS-2 module should not aim at a particular solution, but rather provide as many abstractions as possible to allow for different alternative implementations without changing the main core. The presented 802.16 module is an example of how it is possible to abstract from a particular PHY, BS scheduler, SS UL scheduler, contention resolution mechanism, etc. A highly modular architecture also allows for flexible testing of various radio resource management algorithms. It creates unlimited possibilities for future research in areas, such as $802.16 \mathrm{j}$ and $802.16 \mathrm{~m}$.

\section{ACKNOWLEDGMENTS}

We express our acknowledgments to the following people who helped during development, took part in testing and provided valuable comments on implementation and simulation techniques: Juha Karhula, Levente Sajo, Timo Joensuu, Jani Lakkakorpi, Jani Moilanen, Thomas Höhne, Lauri Kuuru, Yanqun Le, Wu Yi, Zhang Dongmei, Jan Suumäki, Michael Gundlach, Milton Mendieta, Alexey Dudkov, Nenad Veselenovic.

Special thanks for the WiMAX Forum Application Working Group members Tom Tofigh, Shayem Parekh, and Raj Jain for supporting the simulation methodology work item.

\section{REFERENCES}

[1] Wireless LAN medium access control (MAC) and physical layer (PHY) specifications. IEEE Standard 802.11, 1999.

[2] Air interface for fixed broadband wireless access systems. IEEE Standard 802.16, Jun 2004.

[3] Air interface for fixed broadband wireless access systems - amendment for physical and medium access control layers for combined fixed and mobile operation in licensed bands. IEEE Standard 802.16e, Dec 2005.

[4] Harmonized contribution on $802.16 \mathrm{j}$ (mobile multi-hop) usage models. IEEE 802.16 Broadband Wireless Access Working Group, Sep 2006.

[5] Multi-hop relay system evaluation methodology (channel model and performance metrics). IEEE 802.16 Broadband Wireless Access Working Group, Feb 2007.

[6] Air interface for broadband wireless access systems. IEEE Standard 802.16 (Rev2 D8), Dec 2008.

[7] IEEE $802.16 \mathrm{~m}$ evaluation methodology document (EMD). IEEE 802.16 Broadband Wireless Access Group, Mar 2008. 
[8] WiMAX Forum Network Architecture, Sep 2008. Release 1, Version 1.3.0.

[9] Air interface for broadband wireless access systems: Multihop relay specification. IEEE Standard 802.16j (D9), Feb 2009.

[10] Relay SG features. WiMAX Forum, Technical Working Group, Jul 2009.

[11] WiMAX Forum Mobile System Profile Specification: Release 1.5 (Revision 0.2.1), Feb 2009.

[12] O. Alanen. Multicast polling and efficient VoIP connections in IEEE 802.16 networks. In The 10th ACM/IEEE International Symposium on Modeling, Analysis and Simulation of Wireless and Mobile Systems, pages 289294, Oct 2007.

[13] N. Baldo, F. Maguolo, M. Miozzo, M. Rossi, and M. Zorzi. NS-2 MIRACLE: a modular framework for multi-technology and cross-layer support in network simulator 2. In NS tools, Oct 2007.

[14] L. Betancur, R. C. Hincapie, and R. Bustamante. WiMAX channel - PHY model in network simulator 2 . In Workshop on NS-2, Oct 2006.

[15] T. Bohnert, J. Jakubiak, M. Katz, Y. Koucheryavy, E. Monteiro, and E. Borcoci. On evaluating a WiMAX access network for isolated research and data networks using NS-2. In 7th International Conference on Next Generation Teletraffic and Wired/Wireless Advanced Networking, pages 133-147, Sep 2007.

[16] J. Chen, C.-C. Wang, F. C.-D. Tsai, C.-W. Chang, S.S. Liu, J. Guo, W.-J. Lien, J.-H. Sum, and C.-H. Hung. The design and implementation of WiMAX module for NS-2 simulator. In Workshop on NS-2, Oct 2006.

[17] Q. Chen, F. Schmidt-Eisenlohr, D. Jiang, M. TorrentMoreno, L. Delgrossi, and H. Hartenstein. Overhaul of IEEE 802.11 modeling and simulation in NS-2. In The 10th IEEE/ACM International Symposium on Modeling, Analysis and Simulation of Wireless and Mobile Systems, Oct 2007.

[18] J.-F. Cheng. Coding performance of Hybrid ARQ schemes. IEEE Transactions on Communication, 54(6):1017-1029, Jun 2006.

[19] C. Cicconatti, L. Lenzini, and E. Mingozi. Quality of service support in IEEE 802.16 networks. IEEE Networks, 20(2):50-55, Mar/Apr 2006.
[20] A. Dudkov and A. Sayenko. Orthogonal bandwidth request codes for IEEE 802.16 networks. In The 11th International Symposium on Wireless Personal Multimedia Communications, Sep 2008.

[21] F. Frederiksen and T. Kolding. Performance and modeling of WCDMA/HSDPA transmission/H-ARQ schemes. In IEEE Vehicular Technology Conference, pages 472-476, Sep 2002.

[22] P. Frenger, S. Parkvall, and E. Dahlman. Performance comparison of HARQ with chase combining and incremental redundancy in HSDPA. In IEEE Vehicular Technology Conference, pages 1829-1833, Oct 2001.

[23] S. Kim and I. Yeom. IEEE 802.16 simulator. http://cnlab.kaist.ac.kr/802.16/ ieee802.16.html.

[24] T. Kwon, H. Lee, S. Choi, J. Kim, D.-H. Cho, S. Cho, S. Yun, W.-H. Park, and K. Kim. Design and implementation of a simulator based on a cross-layer protocols between MAC and PHY layers in a WiBro compatible IEEE 802.16e OFDMa system. IEEE Communications Magazine, 43(12):136-146, Dec 2005.

[25] J. Lakkakorpi and A. Sayenko. Uplink VoIP delays in IEEE 802.16e using different ertPS resumption mechanisms. In UBICOMM, 2009. accepted for publication.

[26] J. Lakkakorpi, A. Sayenko, J. Karhula, O. Alanen, and J. Moilanen. Active queue management for reducing downlink delays in WiMAX. In Vehicular Technology Conference, 2007. VTC-2007 Fall. 2007 IEEE 66th, pages $326-330$, Sep 2007.

[27] J. Lakkakorpi, A. Sayenko, and J. Moilanen. Comparison of different scheduling algorithms for WiMAX base station: deficit round robin vs. proportional fair vs. weighted round robin. In IEEE WCNC, pages 19911996, Mar/Apr 2008.

[28] E. Lang, S. Redana, and B. Raaf. Business impact of relay deployment for coverage extension in 3GPP LTEAdvanced. In IEEE International Conference on Communication, Jun 2009.

[29] K. Lu, Y. Qian, H.-H. Chen, and S. Fu. WiMAX neworks: from access to service platform. IEEE Network, 22(3):38-45, May/Jun 2008.

[30] H. Martikainen, O. Alanen, and A. Sayenko. ARQ parameters for VoIP in IEEE 802.16 networks. In Wireless Telecommunications Symposium, Apr 2009. 
[31] H. Martikainen, A. Sayenko, O. Alanen, and V. Tykhomyrov. Optimal MAC PDU size in IEEE 802.16. In 4th International Telecommunication Networking Workshop on QoS in Multiservice IP Networks, pages 66-71, Feb 2008.

[32] M. Mendieta. Modelling of test specifics for multivendor WiMAX networks. Master's thesis, Mannheim University of Applied Sciences, 2008.

[33] I. C. Msadaa, F. Filali, and F. Kamoun. An 802.16 model for NS-2 simulator with an integrated QoS architecture. In Simutools, Mar 2008.

[34] S. W. Peters and R. W. Heath. The future of WiMAX: multihop relaying with IEEE 802.16j. IEEE Communications Magazine, Jan 2009.

[35] A. Puchko, V. Tykhomyrov, and H. Martikainen. Link adaptation thresholds for the IEEE 802.16 base station. In Workshop on NS-2 simulator, Oct 2008.

[36] R. J. Punnoose, P. V. Nikitin, and D. D. Stancil. Efficient simulation of ricean fading within a packet simulator. In IEEE Vehicular Technology Conference, pages 764767, Sep 2000.

[37] R. Rouil. The network simulator NS-2 NIST add-on: IEEE 802.16 model (MAC+PHY). Technical report, Jun 2007.

[38] A. Sayenko, O. Alanen, and T. Hämäläinen. Adaptive contention resolution for VoIP services in IEEE 802.16 networks. In The 8th IEEE International Symposium on a World of Wireless, Mobile and Multimedia Networks, Jun 2007.

[39] A. Sayenko, O. Alanen, and T. Hämäläinen. Adaptive contention resolution parameters for the IEEE 802.16 networks. In International Conference on Heterogeneous Networking for Quality, Reliability, Security and Robustness, Aug 2007.

[40] A. Sayenko, O. Alanen, and T. Hämäläinen. ARQ aware scheduling for the IEEE 802.16 base station. In IEEE International Conference on Communication, pages 2667-2673, May 2008.

[41] A. Sayenko, O. Alanen, and T. Hämäläinen. Scheduling solution for the IEEE 802.16 base station. Computer Networks, 52:96-115, 2008.

[42] A. Sayenko, O. Alanen, J. Karhula, and T. Hämäläinen. Ensuring the QoS requirements in 802.16 scheduling. In The 9th IEEE/ACM International Symposium on Modeling, Analysis and Simulation of Wireless and Mobile Systems, pages 108-117, Oct 2006.
[43] A. Sayenko, H. Martikainen, and A. Puchko. Performance comparison of HARQ and ARQ mechanisms in IEEE 802.16 networks. In The 11th ACM/IEEE International Symposium on Modeling, Analysis and Simulation of Wireless and Mobile Systems, pages 411-416, Oct 2008.

[44] H. L. V. Trees. Detection, Estimation and Modulation Theory. Part 1: Detection, Estimation, and Linear Modulation Theory. John Wiley and Sons, 2001.

[45] V. Tykhomyrov, A. Sayenko, H. Martikainen, and O. Alanen. Analysis and performance evaluation of the IEEE 802.16 ARQ mechanism. Journal of communications software and systems, 4(1):29-40, Mar 2008.

[46] V. Tykhomyrov, A. Sayenko, H. Martikainen, O. Alanen, and T. Hämäläinen. On ARQ feedback intensity of the IEEE 802.16 ARQ mechanism. In International Conference on Telecommunications, Jun 2008.

[47] UCB/LBNL/VINT. Network simulator ns-2. http: //www.isi.edu/nsnam/ns. 


\section{OTcl simulation script}

This script introduces the scenario used for modeling WiMAX network during the simulations 


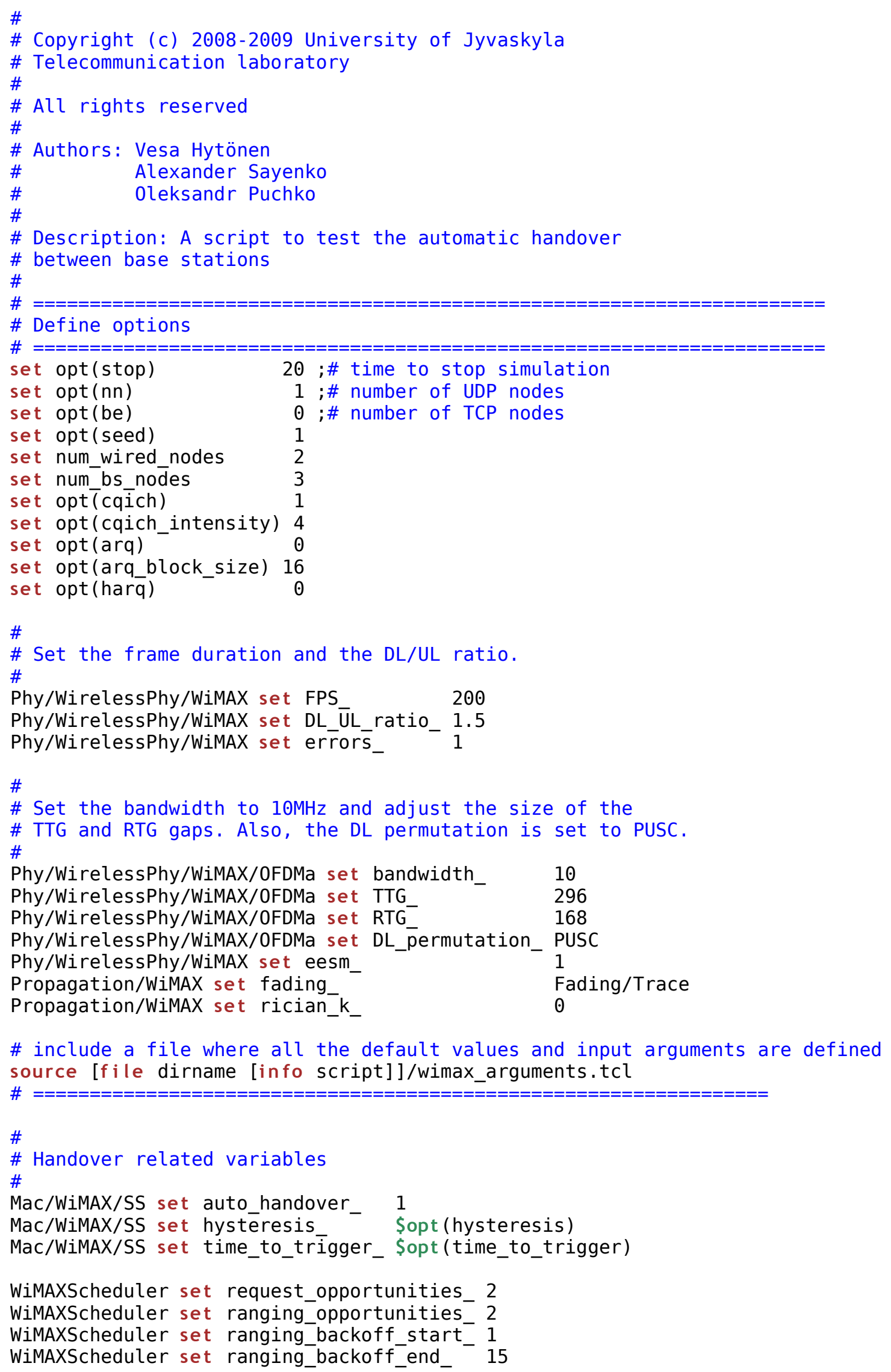




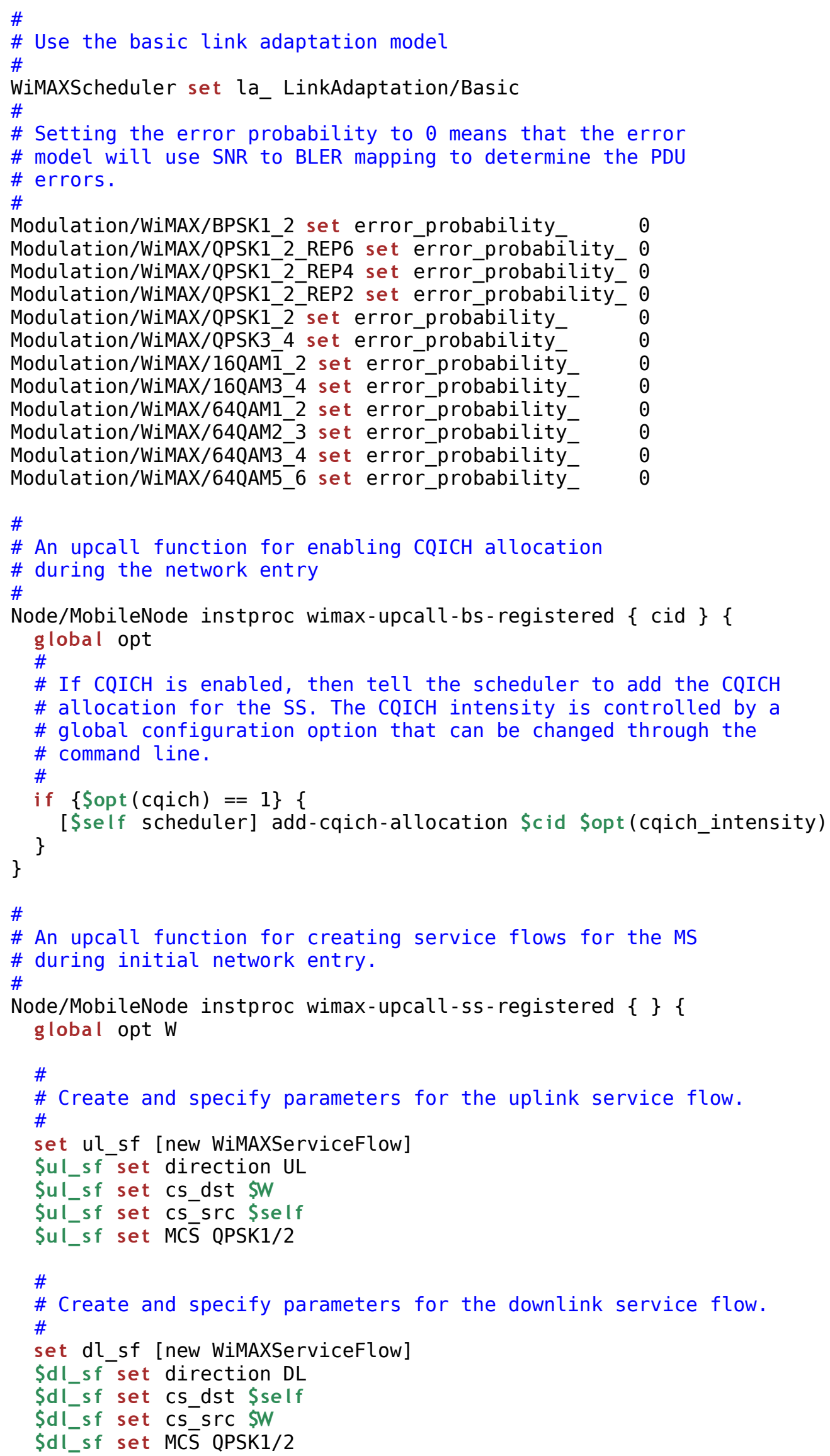




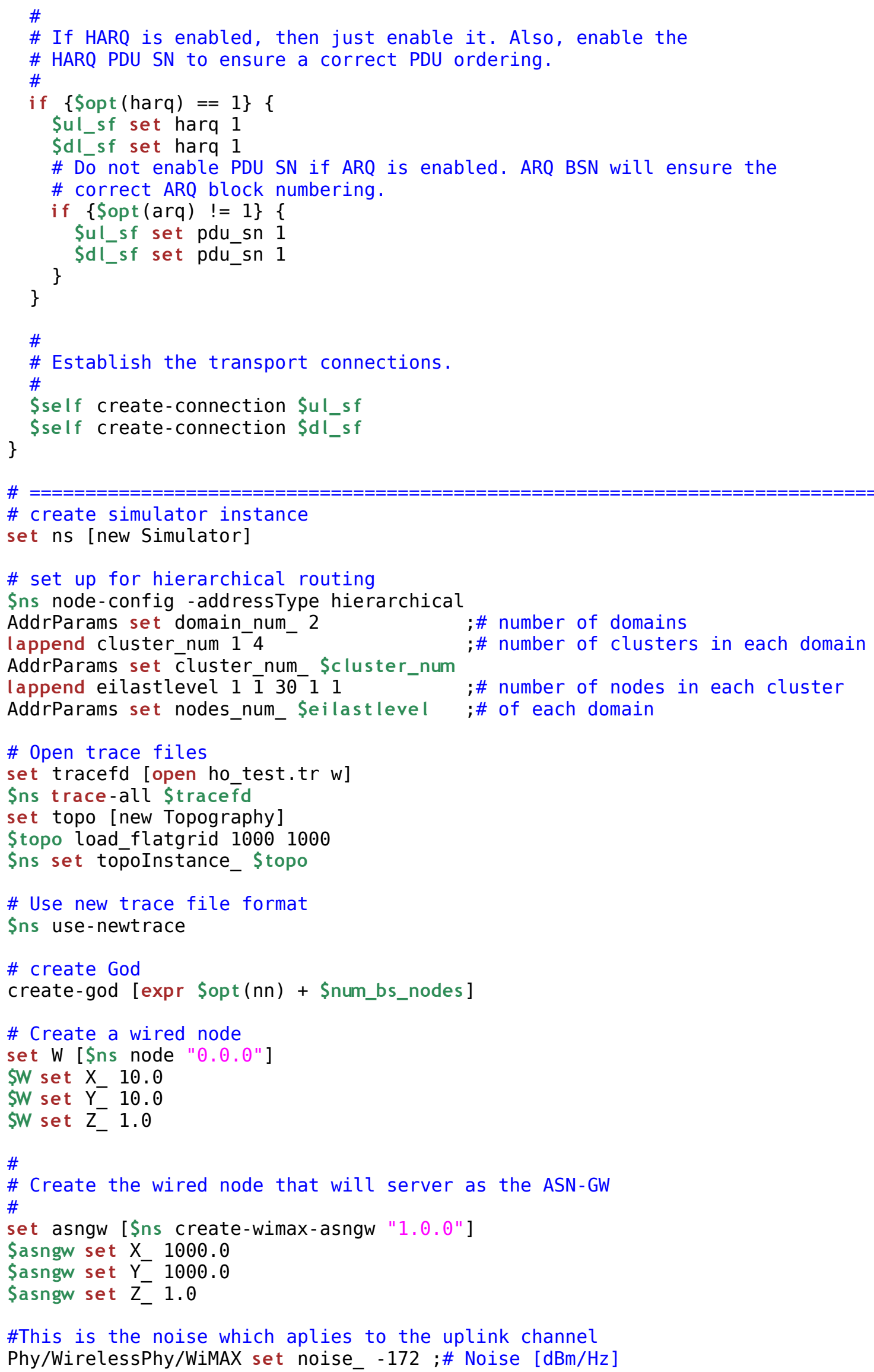




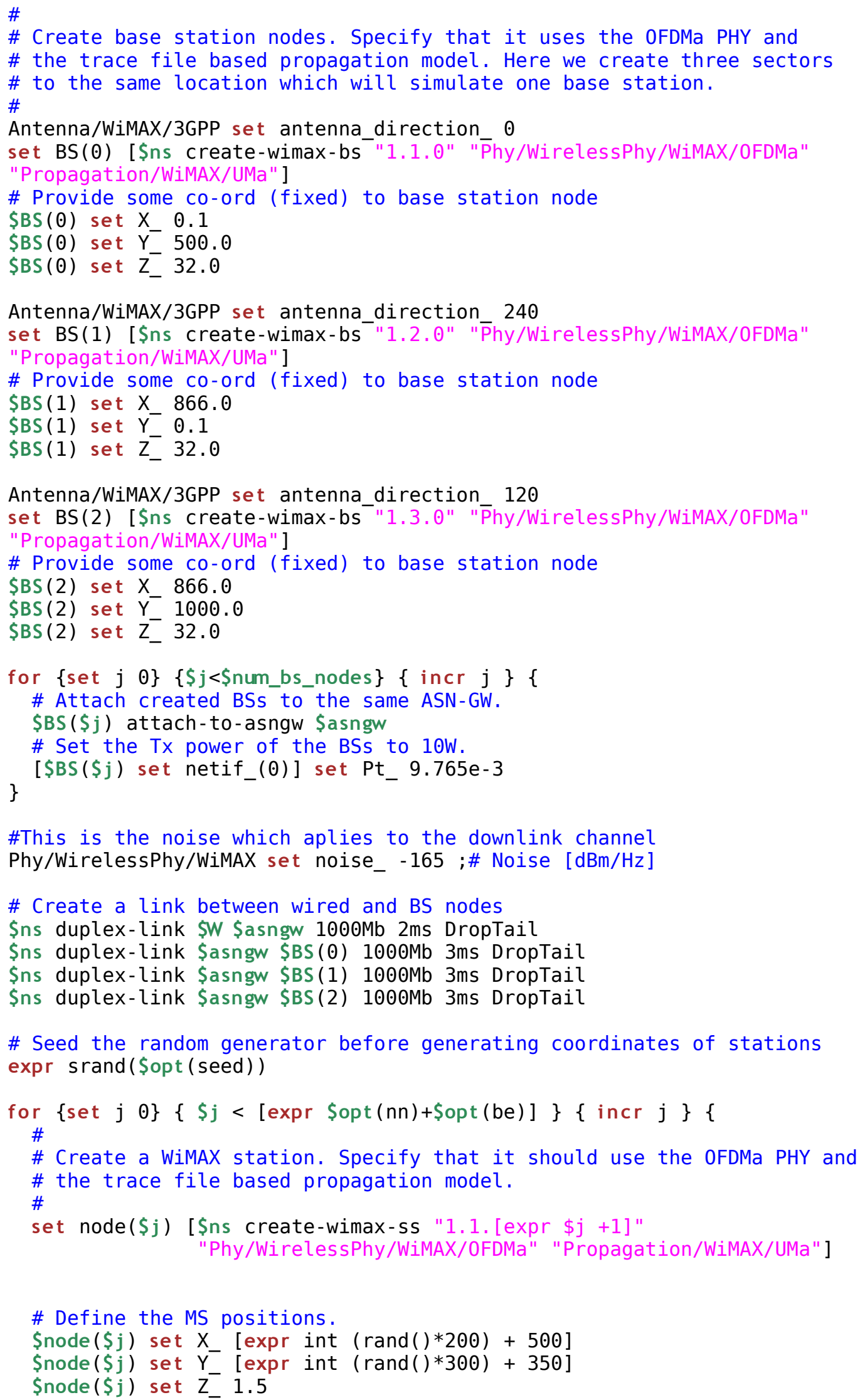




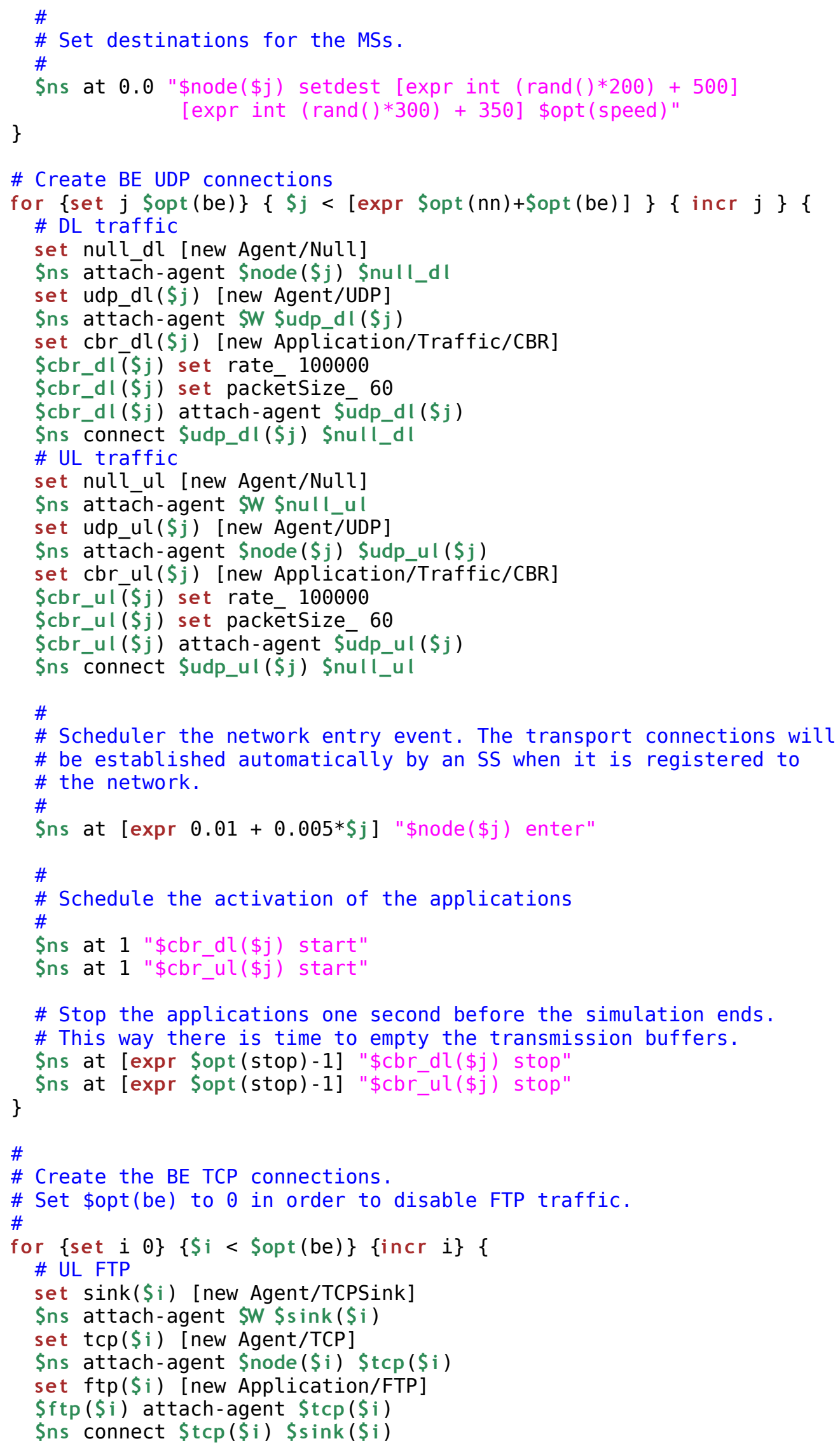




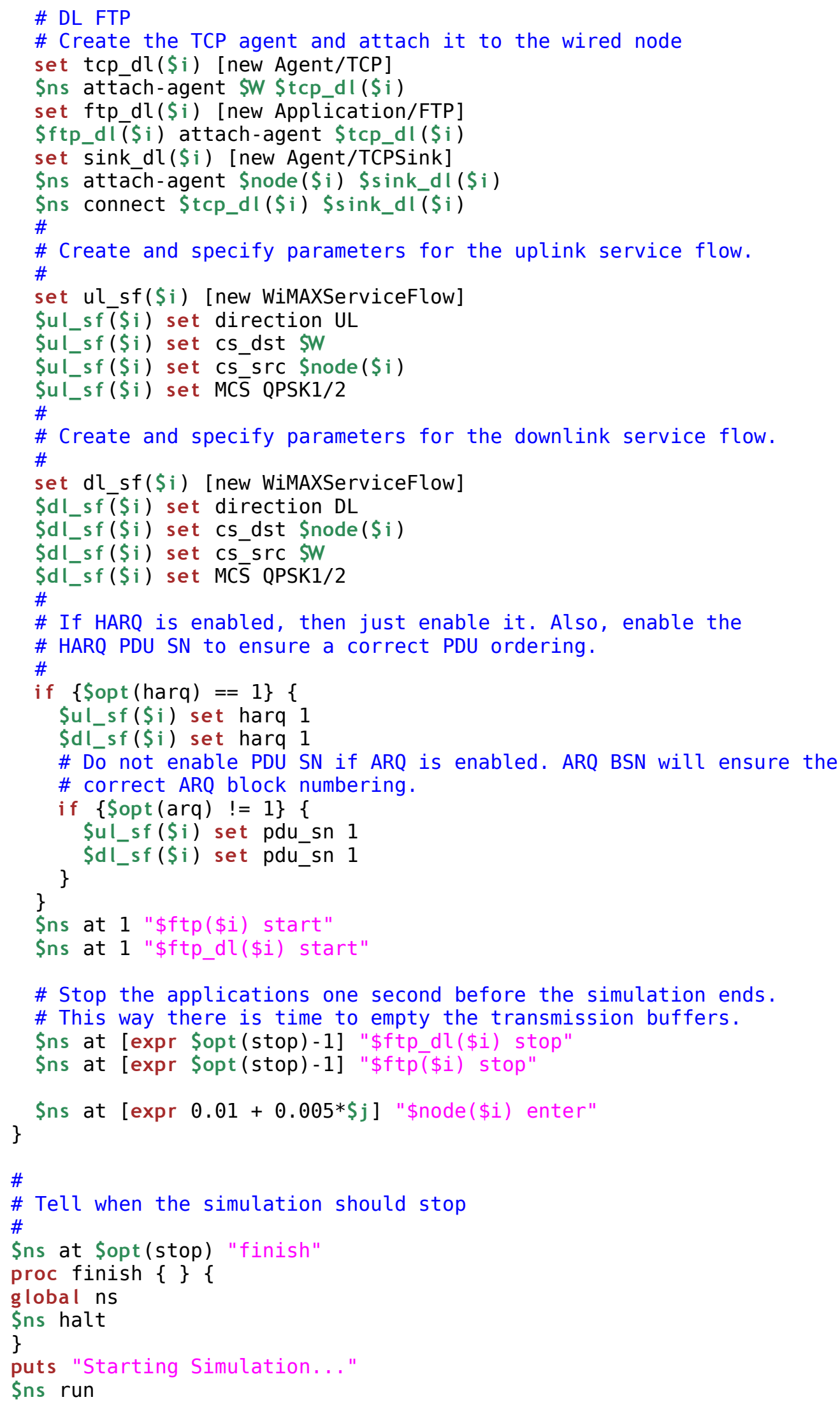

